Minimal tensors and purely electric or magnetic spacetimes of arbitrary dimension

This article has been downloaded from IOPscience. Please scroll down to see the full text article.

2013 Class. Quantum Grav. 30165014

(http://iopscience.iop.org/0264-9381/30/16/165014)

View the table of contents for this issue, or go to the journal homepage for more

Download details:

IP Address: 157.193.117.112

The article was downloaded on 06/09/2013 at 16:51

Please note that terms and conditions apply. 


\title{
Minimal tensors and purely electric or magnetic spacetimes of arbitrary dimension
}

\author{
Sigbjørn Hervik ${ }^{1}$, Marcello Ortaggio $^{2}$ and Lode Wylleman ${ }^{1,3,4}$ \\ ${ }^{1}$ Faculty of Science and Technology, University of Stavanger, N-4036 Stavanger, Norway \\ ${ }^{2}$ Institute of Mathematics, Academy of Sciences of the Czech Republic, Žitná 25, \\ 11567 Prague 1, Czech Republic \\ ${ }^{3}$ Department of Mathematical Analysis, Ghent University, B-9000 Gent, Belgium \\ ${ }^{4}$ Mathematical Institute, Department of Mathematics, Utrecht University, 3584 CD Utrecht, \\ The Netherlands \\ E-mail: sigbjorn.hervik@uis.no,ortaggio@math.cas.cz and lode.wylleman@ugent.be
}

Received 28 December 2012, in final form 13 June 2013

Published 26 July 2013

Online at stacks.iop.org/CQG/30/165014

\begin{abstract}
We consider time reversal transformations to obtain twofold orthogonal splittings of any tensor on a Lorentzian space of arbitrary dimension $n$. Applied to the Weyl tensor of a spacetime, this leads to a definition of its electric and magnetic parts relative to an observer (defined by a unit timelike vector field $\mathbf{u})$, in any dimension. We study the cases where one of these parts vanishes in detail, i.e., purely electric (PE) or magnetic (PM) spacetimes. We generalize several results from four to higher dimensions and discuss new features of higher dimensions. For instance, we prove that the only permitted Weyl types are $\mathrm{G}, \mathrm{I}_{i}$ and $\mathrm{D}$, and discuss the possible relation of $\mathbf{u}$ with the Weyl aligned null directions (WANDs); we provide invariant conditions that characterize PE/PM spacetimes, such as Bel-Debever-like criteria, or constraints on scalar invariants, and connect the PE/PM parts to the kinematic quantities of $\mathbf{u}$; we present conditions under which direct product spacetimes (and certain warps) are PE/PM, which enables us to construct explicit examples. In particular, it is also shown that all static spacetimes are necessarily PE, while stationary spacetimes (such as spinning black holes) are in general neither PE nor PM. Whereas ample classes of PE spacetimes exist, PM solutions are elusive; specifically, we prove that PM Einstein spacetimes of type D do not exist, in any dimension. Finally, we derive corresponding results for the electric/magnetic parts of the Riemann tensor, which is useful when considering spacetimes with matter fields, and moreover leads to first examples of PM spacetimes in higher dimensions. We also note in passing that PE/PM Weyl (or Riemann) tensors provide examples of minimal tensors, and we make the connection hereof with the recently proved alignment theorem (Hervik 2011 Class. Quantum Grav. 28 215009). This in turn sheds new light on the classification of the Weyl tensors based on null alignment, providing a further invariant characterization
\end{abstract}


that distinguishes the (minimal) types G/I/D from the (non-minimal) types II/III/N.

PACS numbers: 04.50. $-\mathrm{h}, 04.20 .-\mathrm{q}$

\section{Contents}

1. Introduction 2

2. Minimal tensors 4

2.1. Definition and algebraic criterion 4

2.2. Sufficient conditions and examples 6

2.3. Minimal tensors and null alignment type $\quad 8$

3. The Weyl tensor: purely electric (PE) or magnetic (PM) spacetimes 9

3.1. Electric and magnetic parts $\quad 10$

3.2. $\mathrm{PE} / \mathrm{PM}$ condition at a point 11

3.3. PE/PM Weyl bivector operators $\quad 12$

$\begin{array}{ll}\text { 3.4. Null alignment properties } & 14\end{array}$

3.5. PE spacetimes 18

3.6. PM spacetimes 26

4. The Ricci and Riemann tensors: Riemann purely electric (RPE) or magnetic (RPM) spacetimes

4.1. Definitions and PE/PM conditions 29

4.2. Null alignment types $\quad 32$

4.3. Direct products and explicit examples 33

5. Conclusion and discussion 35

Acknowledgments 36

Appendix A. Orbits of tensors, Cartan involutions, and null alignment theory 36

A.1. Orbits of tensors; tensors characterized by their invariants 36

A.2 Cartan involutions of the Lorentz group 38

A.3. Null alignment theory 41

Appendix B. Minimal Ricci- and Maxwell-like tensors 43

B.1. Ricci-like tensors 44

B.2. Maxwell-like tensors 45

Appendix C. Timelike unit vector fields: expansion, rotation, shear, and Raychaudhuri equation $\quad 46$

$\begin{array}{lr}\text { References } & 48\end{array}$

\section{Introduction}

Decompositions of tensors relative to an observer (identified here with its normalized timelike 4 -velocity u) are of great import in contemporary theoretical physics. One of the most well-known insights, coming along with Einstein's Special Relativity already, is that the separate electric and magnetic (henceforth also abbreviated to EM) fields in Maxwell's electromagnetism are in fact the electric and magnetic parts, relative to an observer $\mathbf{u}$, of one unified object, the Maxwell tensor $F_{a b}$. Conversely, given a Maxwell tensor and any observer $\mathbf{u}$, one may split the tensor into its electric and magnetic parts relative to $\mathbf{u}$. Although the precise value of the EM components clearly depends on the observer's frame of reference, the property of a field of being (or not), e.g., purely electric (PE) or purely magnetic (PM) 
is in fact intrinsic and can be easily determined using the two Lorentz invariants $F_{a b} F^{a b}$ and $F_{a b} F^{* a b}$ (see [2]). Furthermore, when one considers the electromagnetic field generated by an isolated, bounded source, the associated conserved charges can be computed, via Gauss' law, as specific surface integrals at infinity, to which only the leading (Coulomb) terms of the corresponding electric and magnetic parts will contribute.

As the twofold EM splitting can be performed pointwise at any event and for any $\mathbf{u}$, the procedure applies in General Relativity as well. Given that the latter explains the gravitational interaction through the curved spacetime structure, one may ask whether gravitational quantities exist playing a role analogous to the EM fields in classical electromagnetism, and whether a PE or PM gravitational field can be given an intrinsic meaning and an invariant characterization. Matte [3] showed that the answer to the first question is affirmative, by introducing the electric and magnetic parts, relative to an observer $\mathbf{u}$, of the Riemann tensor of a vacuum metric. For general energy-momentum content (Ricci tensor) this generalizes to the EM parts of the Weyl tensor. In terms of these parts, the decomposed trace-free second Bianchi identities indeed take a form analogous to Maxwell's equations (see, e.g., [4]). A positive answer to the second question was supplied by the work of McIntosh et al [5], who deduced an invariant criterion for deciding whether a given Weyl tensor has PE or PM character (see remark 3.8 below). In addition, building on the analogy with the electromagnetic field, the EM decomposition of the Weyl tensor has proven to be a very useful and, by now, standard tool in the initial-value formulation of the gravitational field, as well as in the definition of conserved charges and asymptotic symmetries (see, e.g., [6-8] and references therein) ${ }^{5}$. It has also played an important role in the study of cosmological models [4, 9].

With the emergence of higher-dimensional physical theories such as string theory, the interest in general $n$-dimensional spacetimes with Lorentzian signature has grown rapidly. In this paper we propose a general viewpoint to the splitting of tensors, deduced from the theory of Cartan involutions of a semi-simple Lie group. In the case of the Lorentz group, these involutions are simply reflections of unit timelike vectors ( $n$-velocities), $\mathbf{u} \mapsto-\mathbf{u}$. As we will see this leads to a twofold splitting of any tensor (see [10,11] and remark 3.2(b) for a comparison with Senovilla's approach). When applied to the Weyl tensor, the splitting provides a natural definition of its electric and magnetic parts relative to $\mathbf{u}$. We show that this definition is sound, by proving that several four-dimensional results concerning PE or PM Weyl tensors or spacetimes generalize to higher dimensions. In addition to Senovilla's papers mentioned above, a similar splitting of the Weyl tensor in higher dimensions has also been considered in the study of asymptotic properties at spatial infinity and of conserved charges, see, e.g., $[12,13]$. Our work does not overlap with the results of such references.

Recently, one of us proved the alignment theorem, stating that (direct sums of) tensors which are not characterized by their invariants are precisely the ones of aligned type II or more special, but not D [1]. In this paper we stress another equivalent fact, in the realm of the splitting relative to $\mathbf{u}$ : such tensors are precisely the ones which do not have a minimal tensor relative to $\mathbf{u}$, in their orbit under the (active) Lorentz group action on tensors (see below). As we will see, if a tensor equals one of its parts in the splitting w.r.t. $\mathbf{u}$, it is itself minimal w.r.t. $\mathbf{u}$ (but not vice versa, in general). In particular, a Weyl tensor which is PE/PM w.r.t. a unit timelike vector $\mathbf{u}$ is minimal w.r.t. the same $\mathbf{u}$, but more stringent conditions than those based on the alignment theorem will be deduced.

The structure of the paper is as follows. Preliminary basic results and definitions necessary for our work, such as theorems about tensors characterized by their invariants, the twofold splitting of a tensor relative to an arbitrary unit timelike vector $\mathbf{u}$, and null alignment theory,

5 It should be noted that, in this context, the EM splitting is sometimes meant w.r.t. a spacelike vector field [7, 8]. 
are relegated to appendix A, since mostly known-however, this can be the starting section for a reader not familiar with such concepts. In section 2 we present an algebraic criterion for a tensor to be minimal w.r.t. $\mathbf{u}$, provide sufficient conditions and examples, and make the connection with the alignment theorem of [1]. The twofold splitting is applied to the Weyl tensor in section 3, and to the Ricci and Riemann tensors in section 4. In both parts we derive several useful results and examples of spacetimes for which the tensors in question are PE or PM. We end with conclusions and a discussion. In appendix B we present an alternative, more explicit proof of the general proposition 2.7 for the special case of Ricci- and Maxwell-like rank 2 tensors (namely: they are minimal if and only if they are not of alignment type II (but not D) or more special). In appendix $\mathrm{C}$ we summarize standard definitions of the kinematic quantities of a unit timelike congruence, and write parts of the Riemann and Weyl tensors in terms of these.

Notation. The symbol $\mathcal{F}_{M}$ denotes the set of smooth scalar functions of an $n$-dimensional spacetime $M$. We will write $A^{\perp}$ for the orthogonal complement of a set $A$, and denote a tensor either in index-free notation $(T)$ or abstract index notation, with lowercase, possibly numbered Latin letters $a, a_{1}, a_{2}, b, c, d, \ldots\left(T^{\alpha_{1} \cdots \alpha_{r}} \beta_{1} \cdots \beta_{s}\right)$, or clumping the abstract indices $\left(T^{\mathbf{a}} \mathbf{b}\right)$, whatever is more convenient in the context. In the index-free notation a metric tensor in use will be denoted by $\mathbf{g}$; likewise tangent vectors and 1 -forms will be bolded, and $\mathbf{v} \sim \mathbf{w}$ means that $\mathbf{v}$ is proportional to $\mathbf{w}$. The Riemann, Ricci and Weyl tensors of a spacetime will be denoted by $R_{a b c d}, R_{a b} \equiv R^{c}{ }_{a c b}$ and $C_{a b c d}$, respectively, while $\mathcal{R} \equiv R^{a}{ }_{a}$ symbolizes the Ricci scalar.

A component of a tensor $T$ in an unspecified frame $\left\{\mathbf{m}_{\alpha=1, \ldots, n}\right\}$ of tangent space, with dual frame $\left\{\mathbf{m}^{\alpha=1, \ldots, n}\right\}$, is denoted by $T^{\alpha_{1} \cdots \alpha_{r}} \beta_{1} \cdots \beta_{s}$ (or $T_{\beta}^{\alpha}$ ). An orthonormal frame (henceforth, ONF) is of the form $\left\{\mathbf{u}, \mathbf{m}_{i=2, \ldots, n}\right\}$, where we will use the frame label ' $u$ ' for the timelike vector $\mathbf{u}$ (instead of 1$)$ and $i, j, k, \ldots=2, \ldots, n$ for the spacelike frame vectors. When $\mathbf{u}$ is a specific timelike vector we will call any $\operatorname{ONF}\left\{\mathbf{u}, \mathbf{m}_{i=2, \ldots, n}\right\}$ a $\mathbf{u}-O N F$. In general $\left(T^{\mathrm{sp}}\right)_{a \cdots b} \equiv h_{a}{ }^{c} \cdots h_{b}{ }^{d} T_{c \cdots d}$ denotes the purely spatial part of a tensor $T$ w.r.t. $\mathbf{u}$ (see (A.9) for the definition of the projector $h_{a}{ }^{c}$ ); if $T=T^{\mathrm{sp}}$ the tensor is called purely spatial (relative to $\mathbf{u}$ ), and in any $\mathbf{u}-\mathrm{ONF}$ only components $T_{i j \ldots}$ can be non-zero for such.

When also one of the spacelike vectors of a $\mathbf{u}$-ONF is selected or preferred, say $\mathbf{m}_{2}$, we shall indicate the remaining labels with $\hat{i}, \hat{j}, \hat{k}, \ldots=3, \ldots, n$ instead. The null vectors

$$
\ell=\frac{\mathbf{u}+\mathbf{m}_{2}}{\sqrt{2}}, \quad \mathbf{n}=\frac{-\mathbf{u}+\mathbf{m}_{2}}{\sqrt{2}},
$$

are normalized by $l^{a} n_{a}=1$ and generate the respective null directions of the timelike plane spanned by $\mathbf{u}$ and $\mathbf{m}_{2}$. The null frame $\left\{\mathbf{m}_{0}=\boldsymbol{\ell}, \mathbf{m}_{1}=\mathbf{n}, \mathbf{m}_{\hat{i}=3, \ldots, n}\right\}$ will then be called adapted to the $\mathbf{u}-\mathrm{ONF}\left\{\mathbf{u}, \mathbf{m}_{i=2, \ldots, n}\right\}$; notice that we use the frame labels ' 0 ' for $l^{a}$ and ' 1 ' for $n^{a}$ (the dual frame consisting of $m^{0}{ }_{a}=n_{a}, m^{1}{ }_{a}=l_{a}$ and $\left.m_{a}^{\hat{i}}=\left(m_{\hat{i}}\right)_{a}, \hat{i}=3, \ldots, n\right)$.

\section{Minimal tensors}

\subsection{Definition and algebraic criterion}

Given the full Lorentz group $G=O(1, n-1)$, the definition of a Cartan involution $\theta$ and of the associated Euclidean product (cf (A.22)) and norm are recalled in section A.2 in the appendix. Now, w.r.t. the Euclidean product associated to the Cartan involution $\theta$, the standard definition of a minimal vector of a tensor space $V$ is the following.

Definition 2.1. A vector (tensor) $T \in V$ is called minimal if $\|g(T)\| \geqslant\|T\|$, for all $g \in G$. 
Since the norm $\|$.$\| is K$-invariant such a minimal tensor is not necessarily unique; i.e., if $T$ is minimal, so is $k(T)$ for $k \in K$. Moreover, for a tensor $T$ the property of being minimal obviously depends on the norm $\|$.$\| and thus on the choice of \theta$ (i.e., of $\mathbf{u}$ ).

An algebraic criterion for when a tensor is minimal was given in [14]. Let us specify it to our situation, culminating to proposition 2.2 below.

Recall that for a Lie group, $G$, we can identify the tangent space of the identity element, $T_{1} G$ as its Lie algebra, $\mathfrak{g}$; i.e., $T_{1} G \cong \mathfrak{g}$. Furthermore, there is an analytic map, exp : $\mathfrak{g} \mapsto G$, along with a local inverse $\exp ^{-1}: U \mapsto \mathfrak{g}$, where $U \subset G$ is some neighborhood of the identity $1 \in G$. This map, along with its inverse, enables us to write any element $g \in U$ as $g=\exp (\mathcal{X})$, for some $\mathcal{X} \in \mathfrak{g}$. Moreover, given any $\mathcal{X} \in \mathfrak{g}$ we can generate a one-parameter subgroup of $G$ by $g_{\tau}=\exp (\tau \mathcal{X})$.

In our situation $G=O(1, n-1)$, and we denote the Lie algebra by $\mathfrak{o}(1, n-1)$. Then the action of an element $\mathcal{X} \in \mathfrak{o}(1, n-1)$ on $V$ is defined via the one-parameter subgroup $g_{\tau}=\exp (\tau \mathcal{X})$; explicitly:

$$
\mathcal{X}(T) \equiv \lim _{\tau \rightarrow 0} \frac{1}{\tau}\left[g_{\tau}(T)-T\right] .
$$

If $\left(\mathcal{X}^{\alpha}{ }_{\beta}\right)$ is the representation matrix of $\mathcal{X}$ acting on tangent space w.r.t. a basis $\left\{\mathbf{m}_{\alpha=1, \ldots, n}\right\}$ we get by (A.3):

$\mathcal{X}(T)^{\alpha_{1} \cdots \alpha_{r}}{ }_{\beta_{1} \cdots \beta_{s}}=-\sum_{k=1}^{r} \mathcal{X}_{\alpha_{k}^{\prime}}^{\alpha_{k}} T^{\alpha_{1} \cdots \alpha_{k}^{\prime} \cdots \alpha_{r}} \beta_{1} \cdots \beta_{s}+\sum_{l=1}^{s} \mathcal{X}_{\beta_{l}}^{\beta_{l}^{\prime}} T^{\alpha_{1} \cdots \alpha_{r}} \beta_{1} \cdots \beta_{l}^{\prime} \cdots \beta_{s}$.

Furthermore, we may split $\mathfrak{o}(1, n-1)$ into eigenspaces of $\theta$ :

$$
\mathfrak{o}(1, n-1)=\mathfrak{B} \oplus \mathfrak{K},
$$

where the +1 eigenspace $\mathfrak{K}$ is the Lie algebra of the maximal compact subgroup $K$, while the -1 eigenspace $\mathfrak{B}$ is the vector space consisting of the generators of the boosts in planes through $\mathbf{u}$. Moreover, since the elements $\mathcal{X} \in \mathfrak{o}(1, n-1)$ are antisymmetric w.r.t. the inner product $\boldsymbol{g}$ it follows from remark A.10 that $\mathcal{X}_{+} \in \mathfrak{K}$ and $\mathcal{X}_{-} \in \mathfrak{B}$ are the antisymmetric, respectively, symmetric part of $\mathcal{X}$ w.r.t. the inner product $\langle-,-\rangle$ (cf (12) and (13) regarding the boost generators). Hence, $\langle\mathcal{X}(T), T\rangle=0$ for all $\mathcal{X} \in \mathfrak{K}$. For $\mathcal{X} \in \mathfrak{B}$ this is not necessarily zero, but

Proposition 2.2. A covariant tensor $T \equiv T_{a_{1} \cdots a_{m}}$ is minimal iff

$$
\langle\mathcal{X}(T), T\rangle=0, \quad \forall \mathcal{X} \in \mathfrak{B} .
$$

In a $\mathbf{u}-O N F\left\{\mathbf{m}_{\alpha}\right\}=\left\{\mathbf{m}_{1}=\mathbf{u}, \mathbf{m}_{i=2, \ldots, n}\right\}$ this is equivalent with

$$
\sum_{k=1}^{m} \sum_{\alpha_{1}=1}^{n} \ldots \sum_{\alpha_{m-1}=1}^{n} T_{\alpha_{1} \cdots \alpha_{k-1} i \alpha_{k} \cdots \alpha_{m-1}} T_{\alpha_{1} \cdots \alpha_{k-1} u \alpha_{k} \cdots \alpha_{m-1}}=0, \quad \forall i=2, \ldots, n .
$$

Proof. The criterion (5) was proved in [14] (theorem 4.3) in a more general context. The component form (6) follows straightforwardly from (A.23), (3) and the fact that $\mathfrak{B}$ is spanned by the boost generators $\mathcal{X}_{i}$ in the $\left(\mathbf{u}, \mathbf{m}_{i}\right)$-planes, $\mathcal{X}_{i}^{a b} \equiv 2 u^{[a} m_{i}^{b]}$.

To write (6) in a covariant way one replaces $T_{\ldots i \ldots}$ by $h_{a}^{b} T_{\ldots b \ldots}$ and $T_{\ldots u \ldots}$ by $u^{c} T_{\ldots c \ldots}$ for the free indices $i$ and $u$, but one has to be careful with the $\alpha_{l}$ s since raising $u$ gives a minus sign.

Example 2.3. For covectors $v_{a}$ the criterion (6) becomes simply

$$
v_{i} v_{u}=0, \forall i=2, \ldots, n \quad \Leftrightarrow \quad h_{a}^{b} v_{b}\left(u^{c} v_{c}\right)=0 .
$$


Hence, $\mathbf{v}$ is minimal w.r.t. $\mathbf{u}$ iff, relative to $\mathbf{u}$, it is either purely temporal (i.e., proportional to u) or purely spatial (i.e., orthogonal to $\mathbf{u}$ ).

For symmetric (Ricci-like) rank 2 tensors $R_{a b}=R_{(a b)}$ we get

$0=\frac{1}{2} \sum_{\alpha=1}^{n}\left(R_{i \alpha} R_{u \alpha}+R_{\alpha i} R_{\alpha u}\right)=\sum_{\alpha=1}^{n} R_{i \alpha} R_{u \alpha}=R_{i j} R_{u}{ }^{j}+R_{i u} R_{u u}=R_{i a} R_{u}^{a}+2 R_{u u} R_{i u}$.

Likewise, for antisymmetric (Maxwell-like) rank 2 tensors $F_{a b}=F_{[a b]}$ (6) reduces to

$$
0=F_{i j} F_{u}^{j}=-F_{i a} F_{u}^{a} \quad \text { or } \quad u_{[a} F_{b] c} F_{d}^{c} u^{d}=0,
$$

i.e., $\mathbf{u}$ is an eigenvector of $F^{a}{ }_{b} F^{b}{ }_{c}$.

Finally, for rank 4 tensors $C_{a b c d}$ satisfying the first two parts of the Riemann-like symmetries (A.33) we get

$0=\sum_{\alpha=1}^{n} \sum_{\beta=1}^{n} \sum_{\gamma=1}^{n} C_{i \alpha \beta \gamma} C_{u \alpha \beta \gamma}=C_{i j k l} C_{u}{ }^{j k l}+2 C_{i j k u} C_{u}{ }^{j k}{ }_{u}=C_{i a b c} C_{u}{ }^{a b c}+4 C_{i a b u} C_{u}{ }^{a b}{ }_{u}$.

The above examples already show an interesting analogy in the four cases. Obviously, a covector is minimal iff it is not null (which could be dubbed 'type $\mathrm{N}$ ' in the sense of alignment theory). Rewriting the conditions (8)-(10) in a null frame, one also immediately sees that: if $R_{a b}$ is minimal it cannot be of any of the types II (not D), III and N (i.e., only the types G, I and $\mathrm{D}$ can be minimal); if $F_{a b}$ is minimal it can be neither type II (not D) nor N (i.e., only the types $\mathrm{G}$ and $\mathrm{D}$ can be minimal); if $C_{a b c d}$ is minimal it cannot be of any of the types II (not D), III and $\mathrm{N}$ (i.e., only the types G, I and D can be minimal). One can show that the converse is also true (i.e., the admitted types are also sufficient conditions to ensure minimality) and that, in fact, a more general such result holds for any tensor, as we shall show below in proposition 2.7 (see also appendix B in the case of $R_{a b}$ and $F_{a b}$ ).

\subsection{Sufficient conditions and examples}

Using $\theta$, any tensor $T$ can be split as (see section A.2 in the appendix for more details)

$$
T=T_{+}+T_{-}, \quad T_{ \pm}=\frac{1}{2}[T \pm \theta(T)],
$$

which will be used in the following.

Consider a $\mathbf{u}$-ONF $\mathcal{F}_{u}=\left\{\mathbf{u}, \mathbf{m}_{i=2, \ldots, n}\right\}$. In such a frame any element $\mathcal{X} \in \mathfrak{B}$ acting on $T_{p} M$ is represented by a symmetric matrix of the form:

$$
[\mathcal{X}]_{\mathcal{F}_{u}}=\left(\mathcal{X}_{\beta}^{\alpha}\right)=\left[\begin{array}{llll}
0 & z_{2} & \cdots & z_{n} \\
z_{2} & 0 & \cdots & 0 \\
\vdots & \vdots & & \vdots \\
z_{n} & 0 & \cdots & 0
\end{array}\right] .
$$

In the null frame $\mathcal{F}^{\prime}=\left\{\boldsymbol{\ell}, \mathbf{n}, \mathbf{m}_{\hat{i}=3, \ldots, n}\right\}$ adapted to $\mathcal{F}_{u}$ (see (1)) $\mathcal{X}$ is represented by the symmetric matrix (in $1+1+(n-2)$ block-form):

$$
[\mathcal{X}]_{\mathcal{F}^{\prime}}=\frac{1}{\sqrt{2}}\left[\begin{array}{ccc}
\lambda & 0 & z_{\hat{i}}^{t} \\
0 & -\lambda & -z_{\hat{i}}^{t} \\
z_{\hat{i}} & -z_{\hat{i}} & 0
\end{array}\right], \quad \lambda=\sqrt{2} z_{2}
$$

In what follows boost-weight (b.w.) decompositions will refer to the adapted null frame $\mathcal{F}^{\prime}$, and given a tensor $T$ the collection of its components of b.w. $b$ will be denoted as $(T)_{b}$ (see section A.3 in the appendix, also for the nomenclature regarding (null alignment) types of tensors in the subsequent text). 
Let us split $\mathcal{X} \in \mathfrak{B}$ using a vector space basis $\left\{\mathcal{X}_{B}, \mathcal{X}_{\hat{i}}\right\}$ of $\mathfrak{B}$, where $\mathcal{X}_{B}$ is the generator of the boost (A.24). Hence, equation (13) becomes $\mathcal{X}=\lambda \mathcal{X}_{B}+z_{\hat{i}} \mathcal{X}_{\hat{i}}$. We note that the b.w. decomposition of $T$ is the eigenvalue decomposition with respect to $\mathcal{X}_{B}$ :

$$
\mathcal{X}_{B}(T)=\sum_{b} b(T)_{b}
$$

This may serve as a definition of the b.w. $b$ components of $T:(T)_{b}$ is the eigenvector of $\mathcal{X}_{B}$ with eigenvalue $b$.

The action of $\mathcal{X}_{\hat{i}}$ on an arbitrary tensor $T$ is a bit more complicated, but can be derived from (3) and (13), with $\lambda=0$ and $z_{\hat{k}}=0, \hat{k} \neq \hat{i}$. Also, using (13) we note that $\mathcal{X}_{\hat{i}}$ raises and lowers the b.w. by 1 ; i.e.,

$$
\mathcal{X}_{\hat{i}}\left((T)_{b}\right)=\left(\mathcal{X}_{\hat{i}}\left((T)_{b}\right)\right)_{b-1}+\left(\mathcal{X}_{\hat{i}}\left((T)_{b}\right)\right)_{b+1} .
$$

Since $\langle-,-\rangle$ is bilinear we have

$$
\langle\mathcal{X}(T), T\rangle=\lambda\left\langle\mathcal{X}_{B}(T), T\right\rangle+z_{\hat{i}}\left\langle\mathcal{X}_{\hat{i}}(T), T\right\rangle .
$$

Thus, to check minimality we can consider $\mathcal{X}_{B}$ and $\mathcal{X}_{\hat{i}}$ also separately. Based on these observations we have

Proposition 2.4. Any of the following conditions is sufficient for a tensor $T \in V$ to be minimal:

(1) $T$ is a $\theta$-eigenvector, i.e., $T=T_{+}$or $T=T_{-}$;

(2) $T$ has the b.w. decomposition $T=(T)_{0}$ (and thus is of type $\left.D\right)$.

Proof. (i) was proven in [14]: essentially, for any $T$, we have $\langle\mathcal{X}(T), T\rangle=2\left\langle\mathcal{X}\left(T_{+}\right), T_{-}\right\rangle$, $\mathcal{X} \in \mathfrak{B}$ (using $\mathcal{X}\left(T_{ \pm}\right) \in V_{\mp}$ ). Thus, if $T_{-}=0$ or $T_{+}=0$ the criterion (5) is fulfilled.

(ii) If $T=(T)_{0}$ then (14) implies $\mathcal{X}_{B}(T)=0$, while (15) and $\langle X, Y\rangle=\sum_{b}\left\langle(X)_{b},(Y)_{b}\right\rangle$ give $\left\langle\mathcal{X}_{\hat{i}}(T), T\right\rangle=0$. Thus $\langle\mathcal{X}(T), T\rangle=0$ from (16) and again (5) is fulfilled.

Remark 2.5. The two conditions of proposition 2.4 are only sufficient conditions and they are, in general, independent. An exception to this statement is the special case $V=T_{p} M$, for which $T=T_{ \pm}$is also necessary to be minimal, see (7), and $T=(T)_{0}$ is equivalent to $T=T_{+}$(i.e., $T$ is a spacelike vector). However, if $T$ represents a Maxwell-like tensor (bivector, $\left.T_{a b}=F_{a b}=F_{[a b]}\right)$ we have $F=(F)_{0} \Leftrightarrow F_{u \hat{i}}=0=F_{2 \hat{i}}$, but $F_{u 2}$ and $F_{\hat{i} \hat{j}}$ can be non-zero, so that $F_{+} \neq F \neq F_{-}$, in general (in order for this example to be meaningful, here we assume we are in four or higher dimensions). Similarly, it is easy to see that $F=F_{-}$implies $F=(F)_{0}$ (the direction of $\mathbf{m}_{2}$ being defined by $F_{u i}$ ), whereas $F=F_{+}$can be of type $\mathrm{G}$ if $n$ is odd (see remark B.2 in appendix B for a complete discussion). Moreover, as we shall discuss below (proposition 2.7), all Weyl tensors of type G, I or D contain a minimal Weyl tensor in their orbit, with no need to satisfy either (1) or (2) above.

Example 2.6. As an example of a more generic minimal tensor, choose a tensor $T=$ $(T)_{-2}+(T)_{0}+(T)_{+2}$ where both $(T)_{-2}$ and $(T)_{+2}$ are non-zero ${ }^{6}$. First, consider (13) using $\mathcal{X}_{B}$; then we get $\mathcal{X}_{B}(T)=\sum_{b} b(T)_{b}=-2(T)_{-2}+2(T)_{+2}$. Consequently,

$$
\left\langle\mathcal{X}_{B}(T), T\right\rangle=-2\left\langle(T)_{-2},(T)_{-2}\right\rangle+2\left\langle(T)_{+2},(T)_{+2}\right\rangle,
$$

which is in general not zero. However, by a boost of the frame,

$$
\left\langle\mathcal{X}_{B}(T), T\right\rangle=-2 \mathrm{e}^{-4 \lambda}\left\langle(T)_{-2},(T)_{-2}\right\rangle+2 \mathrm{e}^{4 \lambda}\left\langle(T)_{+2},(T)_{+2}\right\rangle ;
$$

\footnotetext{
6 In fact, with no essential change in the following argument we could more generally also consider a tensor of the form $T=(T)_{-k}+(T)_{0}+(T)_{+k}$, where $k>1$.
} 
therefore, there exists a boost of the frame such that $\left\langle\mathcal{X}_{B}(T), T\right\rangle=0$ in which case $\left\langle(T)_{-2},(T)_{-2}\right\rangle=\left\langle(T)_{+2},(T)_{+2}\right\rangle$.

Consider next (13) using $\mathcal{X}_{\hat{i}}: \mathcal{X}_{\hat{i}}(T)$ has only odd b.w., so $\left(\mathcal{X}_{\hat{i}}(T)\right)_{b}=0$ for $b$ even. Thus, since $T$ has only even b.w. components:

$$
\left\langle\mathcal{X}_{\hat{i}}(T), T\right\rangle=0 .
$$

Thus we reach the conclusion that for any $T=(T)_{-2}+(T)_{0}+(T)_{+2}$ there exists a boost generated by $\mathcal{X}_{B}$ such that it is minimal. For this minimal vector, we have the condition $\left\langle(T)_{-2},(T)_{-2}\right\rangle=\left\langle(T)_{+2},(T)_{+2}\right\rangle$.

We still notice that it is important in this example that both $(T)_{-2}$ and $(T)_{+2}$ are non-zero (alternatively, both zero for which $T=(T)_{0}$ and it falls under the spell of proposition 2.4). Indeed, if one of these parts were zero while the other is not, there would not be any minimal $T$. This is connected to the fact that tensors which are of type II or more special, but not D nor $\mathrm{O}$, do not have a minimal vector in their orbit (see proposition 2.7).

Furthermore, we should emphasize that the minimal example $T=(T)_{-2}+(T)_{0}+(T)_{+2}$ does not need to fulfil condition (1) nor (2) in proposition 2.4 showing, again, that these conditions are only sufficient.

\subsection{Minimal tensors and null alignment type}

In this subsection we revisit the 'alignment theorem' for tensors over a Lorentzian space of any dimension proved in [1], giving a more streamlined proof and adding the connection with minimal tensors. Version A is the contrapositive of version B. The statements (1) assume a chosen unit timelike vector $\mathbf{u}$ and associated Euclidean product, and the abbreviations (Act) and (Pass) refer to the active and passive viewpoints.

Proposition 2.7. For a tensor $T$ the following are equivalent:

(Version A)

(1a) (Act) There exists a minimal tensor $v$ in the orbit $\mathcal{O}(T)$; (Pass) there exists a possibly different vector $\mathbf{u}^{\prime}$ such that the representation $\tilde{T}$ of $T$ in a $\mathbf{u}^{\prime}$-ONF is minimal in $\mathcal{O}(\tilde{T})$.

(2a) $T$ is of type $O, D$, or any other type which is not type II or more special.

(3a) $T$ is characterized by its invariants.

\section{(Version B)}

(1b) (Act) There exists no minimal tensor $v$ in the orbit $\mathcal{O}(T)$; (Pass) no ONFrepresentation $\tilde{T}$ of $T$ is minimal.

(2b) $T$ is of type II or more special, but not $D$ nor $O$.

(3b) $T$ is not characterized by its invariants.

\section{Proof.}

(1a) $\Leftrightarrow$ (3a): let $\mathfrak{M} \subset V$ denote the set of minimal vectors. In [14] it was proved that

$$
\mathfrak{M} \cap \mathcal{O}(\tilde{T}) \neq \emptyset \Leftrightarrow \mathcal{O}(\tilde{T}) \in \mathfrak{C}
$$

and the equivalence follows from corollary A.4. 
$(2 b) \Leftarrow(3 b)$ : from [14] we have that, if $\mathcal{O}(T)$ is not closed, then there exists a vector $v_{0}$ in the closure $\overline{\mathcal{O}(T)}$ and $\mathcal{X} \in \mathfrak{B}$ such that $\mathrm{e}^{\tau \mathcal{X}}(T) \rightarrow v_{0}$, as $\tau \rightarrow+\infty$. By considering the b.w. decomposition with respect to the boost $B(\tau)=\mathrm{e}^{\tau \mathcal{X}}$, we get [1]

$$
\mathrm{e}^{\tau \mathcal{X}}(T)=\sum_{b} \mathrm{e}^{\tau b}(T)_{b}
$$

Since $v_{0}$ is finite we need $b \geqslant 0$, or $b \leqslant 0$. By the isomorphism $b \leftrightharpoons-b$, we can assume $b \leqslant 0$. Moreover, type $\mathrm{D}$ is ruled out by proposition 2.4 , and the result follows.

$(2 b) \Rightarrow(3 b)$ : if the tensor $T$ is of type II or more special, but not type D nor O, then there exists a b.w. decomposition

$$
T=\sum_{b \leqslant 0}(T)_{b}
$$

By the action of the boost $(\tau \rightarrow+\infty)$ :

$$
\mathrm{e}^{\tau \mathcal{X}}(T)=\sum_{b \leqslant 0} \mathrm{e}^{\tau b}(T)_{b} \rightarrow(T)_{0} .
$$

If $v_{0} \equiv(T)_{0}$ is in $\mathcal{O}(T)$, then there exists a frame such that $T=(T)_{0}$, hence type D or O which is a contradiction. Thus $v_{0}$ is not in $\mathcal{O}(T)$, which is consequently not closed, and corollary A. 4 concludes the proof.

Explicitly, in the case of the Weyl tensor condition (2a) covers type G, strict type I (including subtypes such as $\mathrm{I}_{i}$ ), type $\mathrm{D}$ and $\mathrm{O}$, while condition (2b) covers strict type II (not D), III (including subtypes such as $\mathrm{III}_{i}$ ) and $\mathrm{N}$.

The general result remains valid for a collection (or direct sum) of tensors instead of a single one. Here a collection $\left(T_{i}\right)$ is called 'of (aligned) type II or more special' if all $T_{i}$ are of the form (18) in the same null frame. For example, if the Weyl and Ricci tensors of a metric at a spacetime point are both type $\mathrm{N}$ w.r.t. the same null vector, then the corresponding Riemann tensor will be of type $\mathrm{N}$ as well, as follows from proposition A.13. If, however, they are both type $\mathrm{N}$ but w.r.t. different null vectors, then they are not aligned and there is a minimal vector: if $R=(R)_{-2}$ and $C=(C)_{+2}$, then we formally write the Riemann tensor as $T=[R, C]$ and we have $T=(T)_{-2}+(T)_{+2}$ such that we are back in the example considered in section 2.2, $T$ being minimal in a frame such that $\langle R, R\rangle=\langle C, C\rangle$.

In appendix B we give more explicit proofs of proposition 2.7 in the case of vectors and Ricci- or Maxwell-like rank 2 tensors.

\section{The Weyl tensor: purely electric (PE) or magnetic (PM) spacetimes}

In the context of General Relativity and its higher-dimensional extensions, the Weyl tensor is a natural object to consider, e.g., in the classification of exact solutions (in particular, of Einstein spacetimes $R_{a b}=\mathcal{R} g_{a b} / n$ ), in the study of gravitational radiation, of asymptotic properties of spacetimes, etc. Throughout this section, we consider spacetimes of dimension $n \geqslant 4$, and apply the general orthogonal splitting of tensors relative to an observer with timelike vector field $\mathbf{u}$, outlined in section A.2 in the appendix, to the Weyl tensor $C_{a b c d}$ at a spacetime point. This enables us to define PE and PM Weyl tensors and spacetimes, to work out several useful results such as Bel-Debever criteria, the structure of the associated Weyl bivector operator and null alignment properties, and to provide illustrative examples. We will see that several well-known results in four dimensions generalize to arbitrary dimensions. In the next section we shall apply a similar analysis to the Ricci and Riemann tensors, which is relevant in the study of spacetimes which contain matter fields. 


\subsection{Electric and magnetic parts}

As before, we consider a fixed unit timelike vector $\mathbf{u}$ and the corresponding Cartan involution $\theta$.

Definition 3.1. The tensor $\left(C_{+}\right)_{a b c d}\left(\left(C_{-}\right)_{a b c d}\right)$ is called the electric (magnetic) part of the Weyl tensor w.r.t. $\mathbf{u}$.

Recall the definition (equation (A.9)) of the orthogonal projector

$$
h_{a b} \equiv g_{a b}+u_{a} u_{b} .
$$

Define the tensor

$$
E_{a b} \equiv C_{a e b f} u^{e} u^{f}=h_{a}{ }^{c} h_{b}{ }^{d} C_{c e d f} h^{e f},
$$

where (A.34) implies the last equality. Obviously, this is a trace-free symmetric rank 2 tensor which is moreover purely spatial relative to $\mathbf{u}: E_{a b}=\left(E^{\mathrm{sp}}\right)_{a b}$. Using (A.9), (A.13) and the symmetries (A.33) one obtains

$$
\begin{aligned}
& \left(C_{+}\right)^{a b}{ }_{c d}=h^{a e} h^{b f} h_{c}{ }^{g} h_{d}{ }^{h} C_{e f g h}+4 u^{[a} u_{[c} C^{b] e}{ }_{d] f} u_{e} u^{f}=\left(C^{\mathrm{sp}}\right)^{a b}{ }_{c d}+4 u^{[a} u_{[c} E^{b]}{ }_{d]}, \\
& \left(C_{-}\right)^{a b}{ }_{c d}=2 h^{a e} h^{b f} C_{e f k[c} u_{d]} u^{k}+2 u_{k} u^{[a} C^{b] k e f} h_{c e} h_{d f} .
\end{aligned}
$$

In any ONF $\left\{\mathbf{u}, \mathbf{m}_{i=2, \ldots, n}\right\}$ the non-identically vanishing electric (magnetic) part accounts for the components of the Weyl tensor with an even (odd) number of indices $u$ (cf section A.2.1 in the appendix). The first, purely spatial term of the Weyl electric part (20) covers the $C_{i j k l}$ components, of which there are $N_{0}(n)=\left(n^{2}-2 n+4\right)(n+1)(n-3) / 12$ independent ones; the last term covers the $N_{2}(n)=(n+1)(n-2) / 2$ independent $C_{u i u j}$ components; however, the latter are fully determined by the former since

$$
C_{u i u j}=C_{i k j}{ }^{k},
$$

which is the component form of the trace-free property (A.34) also expressed in (19); thus there are $N_{0}(n)-N_{2}(n)=n\left(n^{2}-1\right)(n-4) / 12$ extra independent PE components $C_{i j k l}$ in addition to the $C_{u i u j}$ ones. The Weyl magnetic part (21) has $N_{1}(n)=\left(n^{2}-1\right)(n-3) / 3$ independent components $C_{u i j k}$. Together these add up to the $(n-3) n(n+1)(n+2) / 12$ independent components of the Weyl tensor in $n$ dimensions (see also [11]).

Remark 3.2(a). The already known four-dimensional case $n=4$ has somewhat special properties, which we now briefly review. One has $N_{0}(4)=N_{2}(4)=5$, such that the relations (22) can be inverted to give

$$
C_{i j k l}=2\left(\delta_{i[k} C_{l] u j u}-\delta_{j[k} C_{l] u i u}\right) \quad(n=4) .
$$

Using (19) this reads $\left.\left(C^{\mathrm{sp}}\right)^{a b}{ }_{c d}=4 h_{[c}^{[a} E^{b]} d\right]$ in covariant form. Thus (19) and (20) imply that the tensors $\left(C_{+}\right)_{a b c d}$ and $E_{a b}$, both having five independent frame components, are in biunivocal relation:

$\left.E_{a b}=\left(C_{+}\right)_{a c b d} u^{c} u^{d} \quad \leftrightarrow \quad\left(C_{+}\right)^{a b}{ }_{c d}=4\left(h_{[c}^{[a}+u^{[a} u_{[c}\right) E^{b]} d\right] \quad(n=4)$.

We have $N_{1}(4)=5$ as well. Define

$$
H_{a b} \equiv \frac{1}{2} \varepsilon_{a c e f} C^{e f}{ }_{b d} u^{c} u^{d} \quad(n=4),
$$

where $\varepsilon_{a b c d}$ is the volume element. Just as $E_{a b}, H_{a b}$ is a purely spatial, symmetric and trace-free rank 2 tensor which thus has five independent components. Then, by virtue of the identity 
$\varepsilon^{a b e f} u_{e} \varepsilon_{c d f g} u^{g}=2 h^{a}{ }_{[c} h_{d]}^{b}$ (21) can be rewritten, and $\left(C_{-}\right)_{a b c d}$ and $H_{a b}$ are in biunivocal relation:

$H_{a b} \equiv \frac{1}{2} \varepsilon_{a c e f}\left(C_{-}\right)^{e f}{ }_{b d} u^{c} u^{d} \leftrightarrow\left(C_{-}\right)^{a b}{ }_{c d}=2 \varepsilon^{a b e f} u_{e} u_{[c} H_{d] f}+2 \varepsilon_{c d e f} u^{e} u^{[a} H^{b] f} \quad(n=4)$.

Adding the expressions in (24) and (26) for $C_{+}$and $C_{-}$, one obtains the well-known formula for the Weyl tensor in four-dimensional General Relativity in terms of $E_{a b}$ and $H_{a b}$ [9], which are usually referred to as the electric and magnetic parts of the Weyl tensor. Since they are respectively equivalent with $C_{+}$and $C_{-}$this justifies the above definition of Weyl electric and magnetic parts, for general $n$.

Remark 3.2(b). In [10] and [11] Senovilla proposed a construction for generalizing the electromagnetic decomposition relative to a unit timelike vector $\mathbf{u}$, applicable to any tensor $T$ and based on the consideration of maximal antisymmetric index slots. If the number of such slots is $r$ then one constructs $2^{r}$ different tensors from $T$, by taking for each slot a contraction with either $u^{a}$ (yielding an electric ' $\mathrm{E}$ '-contribution for that slot) or $u_{a} \varepsilon^{a a_{1} \cdots a_{n-1}}$ (yielding a magnetic ' $\mathrm{H}$ '-contribution). However, by the antisymmetry of the slots this is equivalent to contraction (over $b, d, \ldots, f$ ) with $u_{a} u^{b} h_{c}{ }^{d} \cdots h_{e}{ }^{f}$ and $h_{a}{ }^{b} h_{c}{ }^{d} \cdots h_{e}{ }^{f}$, respectively. Then, our $T_{+}\left(T_{-}\right.$) part collects the $2^{r-1}$ tensors constructed in this way with an even (odd) number of E-parts. For instance, when $T_{a[b c]} \neq T_{[a b c]}$ in (A.14)-(A.15) then $r=2$, and the first and second term in the second of (A.14) represent the associated $\mathrm{HH}$ and EE tensors associated to $T_{a[b c]} \neq T_{[a b c]}$ $(r=2)$, respectively, while the second of (A.15) contains respective equivalents of the HE and EH tensors. For the Weyl tensor we also have $r=2$; our magnetic part $C_{-}$corresponds to Senovilla's EH and HE tensors, which are equivalent due to the symmetry $C_{a b c d}=C_{c d a b}$; our electric part $C_{+}$covers the EE and $\mathrm{HH}$ tensors, where the former can be seen as a part of the latter due to (22). Notice that one has a reversed situation for a Maxwell field $F_{a b}$ since $r=1$, i.e., $F_{+}\left(F_{-}\right)$covers the ONF components $F_{i j}\left(F_{u i}\right)$ and is the magnetic (electric) part. As another example, for symmetric rank 2 tensors $T$ like the energy-momentum tensor one has $r=2$, and the electric part, $T_{+}$, then assembles the stress-pressure two-tensor $\left(T_{i j}\right)$ and scalar energy density $\left(T_{u u}\right)$ as measured by $\mathbf{u}$, while the magnetic part $T_{-}$represents the heat flux vector. Equivalently for these situations, the 'electric (magnetic) part' collects the $2^{r-1}$ tensors with an even (odd) number of H-parts. This leads us to the following definition for general tensors, where we thus refer to the definition of H/E-parts in $[10,11]$ and the above explanation.

Definition 3.3. Let $T$ be any tensor with $r$ maximal antisymmetric index slots. The electric (magnetic) part of $T$ relative to a unit timelike vector $\mathbf{u}$ is the collection of the $2^{r-1}$ tensors with an even (odd) number of H-parts; this part equals $T_{+}\left(T_{-}\right)$when $r$ is even, and $T_{-}\left(T_{+}\right)$ when $r$ is odd.

\subsection{PE/PM condition at a point}

Definition 3.4. At a point p, the Weyl tensor $C$ is called purely electric (magnetic) (henceforth, $P E(P M))$ w.r.t. $\mathbf{u}$ if $C=C_{+} \leftrightarrow C_{-}=0\left(C=C_{-} \leftrightarrow C_{+}=0\right)$. If such a $\mathbf{u}$ exists the Weyl tensor is called PE (PM); it is called properly $\mathrm{PE}(\mathrm{PM})$ w.r.t. $\mathbf{u}$ if $C=C_{+} \neq 0\left(C=C_{-} \neq 0\right)$. A spacetime, or an open region thereof, is called (properly) PE (PM) if the Weyl tensor is (properly) PE (PM), everywhere.

In any $\operatorname{ONF}\left\{\mathbf{u}, \mathbf{m}_{i=2, \ldots, n}\right\}$ a non-zero Weyl tensor is PE w.r.t. $\mathbf{u}$ iff $C_{u i j k}=0$, $\forall i, j, k=2, \ldots, n$; in view of (22), it is PM w.r.t. u iff $C_{i j k l}=0, \forall i, j, k, l=2, \ldots, n$. 
In analogy with the Bel-Debever criteria for null alignment [9, 15], and using the properties (A.33) and (A.34), one may rewrite this in the following covariant way.

Proposition 3.5. (Weyl PE/PM Bel-Debever criteria) Let $\mathbf{u}$ be a unit timelike vector,

$$
g^{a b} u_{a} u_{b}=-1
$$

Then a Weyl tensor $C_{a b c d}$ is

- PE w.r.t. u iff

$$
u_{a} g^{a b} C_{b c\left[d_{e}\right.} u_{f]}=0
$$

- PM w.r.t. u iff

$$
u_{[a} C_{b c][d e} u_{f]}=0 .
$$

These Bel-Debever criteria are covariant tensor equations, only involving the metric inverse $g^{a b}$, the Weyl tensor $C_{a b c d}$ and the 1-form $u_{a}$. The big advantage of this format of the $\mathrm{PE} / \mathrm{PM}$ conditions is that one may take any basis $\left\{\mathbf{m}_{\alpha=1, \ldots, n}\right\}$ of $T_{p} M$, with dual basis $\left\{\mathbf{m}^{\alpha=1, \ldots, n}\right\}$ of $T_{p}^{*} M$, and consider the components $g^{\alpha \beta}, C_{\alpha \beta \gamma \delta}$ and $u_{\alpha}$. E.g., when the metric is given in coordinates over a neighborhood of $p$, one may take the corresponding holonomic frames of coordinate vector fields and differentials. One then considers (27) and (28) ((27) and (29)) as a system of quadratic equations in the $n$ unknowns $u_{\alpha}$; if a solution to this system exists then the Weyl tensor is PE (PM) relative to the corresponding $\mathbf{u}$. However, since $n \geqslant 4$ the number $1+N_{1}(n)=(n-2)\left(n^{2}-n-3\right) / 3\left[1+N_{0}(n)=n(n-1)^{2}(n-2) / 12\right]$ of independent equations in this system exceeds $n$, with degree of overdeterminacy $d_{1}(n)=(n-1)\left(n^{2}-2 n-6\right) / 3$ $\left[d_{0}(n)=n\left(n^{3}-4 n^{2}+5 n-14\right) / 12\right]$. For $n=4$ we already have $d_{0}(4)=d_{1}(4)=2$, and we note that $d_{0}(n)-d_{1}(n)=(n+1)(n-2)(n-3)(n-4) / 12$ in general, which increases with $n$. Hence, for a generic metric and Weyl tensor no solution $\mathbf{u}$ to the PE or PM conditions can be found, not even at a point $p$, and for $n>4$ the situation is worse for PM (the number of equations then being quartic in $n$ while cubic in the PE case). However, several special metrics do admit solution(s) $\mathbf{u}$, as discussed in the following. In particular, in the next paragraph we will meet easily computable necessary conditions for the above PE and PM equations to have solutions $\mathbf{u}$. In section 3.4 we will discuss the alignment types for PE/PM Weyl tensors and discuss the uniqueness of solutions $\mathbf{u}$. In section 3.5 we will see that ample classes of PE spacetimes exist, whereas PM spacetimes are most elusive (section 3.6).

Remark 3.6. In the case where only the contraction (19) vanishes $\left(C_{u i u j}=0\right)$, we will say that the Weyl tensor is 'PM' (note that the quotes are part of the name); this is only equivalent to PM for $n=4$, but gives a weaker condition for $n>4$ dimensions (since $C_{i j k l}$ need not vanish).

\subsection{PE/PM Weyl bivector operators}

Consider the real $N$-dimensional vector space $\wedge^{2} T_{p} M$ of contravariant bivectors (antisymmetric tensors $F^{a b}=F^{[a b]}$ ) at $p$, where $N=n(n-1) / 2$. In view of the first three symmetries in (A.33) the map

$$
\text { C: } \quad F^{a b} \mapsto \frac{1}{2} C^{a b}{ }_{c d} F^{c d}=\frac{1}{2} F^{c d} C_{c d}^{a b}
$$

is a linear operator (=endomorphism) of $\wedge^{2} T_{p} M$, referred to as the Weyl bivector operator [16], which is symmetric (self-adjoint) w.r.t. the restriction $\mathrm{g}$ to $\wedge^{2} T_{p} M$ of the inner product $g$ on $\mathcal{T}_{0}^{2}, \operatorname{cf}($ A.16):

$$
\mathrm{g}(\mathrm{C}(\mathrm{F}), \mathrm{G})=\mathrm{g}(\mathrm{F}, \mathrm{C}(\mathrm{G})), \quad \mathrm{F}, \mathrm{G} \in \wedge^{2} T_{p} M .
$$


Consider a unit timelike vector $\mathbf{u}$. Through the tensor structure of bivector space, the corresponding $\theta$ acts on it by $F^{a b} \mapsto \theta^{a}{ }_{c} \theta^{b}{ }_{d} F^{c d}$. We can then repeat the constructions of section A.2 of the appendix replacing $T_{p} M$ by $\wedge^{2} T_{p} M$ and $g$ by $g .{ }^{7}$ In particular, the Weyl bivector operator $\mathrm{C}$ is viewed as a type $(1,1)$ tensor over $\wedge^{2} T_{p} M$ and can be decomposed into its electric and magnetic parts $\mathrm{C}_{ \pm}$, which are also symmetric w.r.t. g. Here, $\mathrm{C}_{ \pm}$are the endomorphisms of $\wedge^{2} T_{p} M$ obtained by replacing $C_{a b c d}$ by $\left(C_{ \pm}\right)_{a b c d}$ in (30). Hence, by remark A.10, $C_{+}$and $C_{-}$are the symmetric and antisymmetric parts of $C$ w.r.t. $\langle-,-\rangle$, respectively. Hence, whereas it is cumbersome to say something general about the eigenvector-eigenvalue structure of Weyl operators (in particular in the type $\mathrm{I} / \mathrm{G}$ case), for $P E\left(\mathrm{C}=\mathrm{C}_{+}\right)$or $P M$ $\left(\mathrm{C}=\mathrm{C}_{-}\right)$Weyl operators, we have the following.

Proposition 3.7. A PE or PM Weyl operator is diagonalizable, i.e., a basis of eigenvectors for $\wedge^{2} T_{p} M$ exists. A PE (PM) Weyl operator has only real (purely imaginary) eigenvalues. Moreover, a PM Weyl operator has at least $s=\frac{(n-1)(n-4)}{2}$ zero eigenvalues ( $s$ being the signature of $\mathrm{g})$.

Proof. The first and second statements follow immediately from the fact that $\mathrm{C}_{+}\left(\mathrm{C}_{-}\right)$are symmetric (antisymmetric) linear operators w.r.t. a Euclidean inner product on $\wedge^{2} T_{p} M$. To make this more explicit and to prove the third statement, consider the ONF

$$
\mathcal{B}=\left\{[u i] \equiv \mathbf{u} \wedge \mathbf{m}_{i},[j k] \equiv \mathbf{m}_{j} \wedge \mathbf{m}_{k}\right\}
$$

of $\wedge^{2} T_{p} M$ induced by the ONF $\left\{\mathbf{u}, \mathbf{m}_{i=2, \ldots, n}\right\}$ of $T_{p} M$. Using a $(p+q)$-block form, where $p=n-1$ and $q=\frac{(n-1)(n-2)}{2}(p \leqslant q$ for $n \geqslant 4)$, the matrix representations of $\theta$ and $\mathrm{C}_{ \pm}$w.r.t. $\mathcal{B}$ are

$$
[\theta]_{\mathcal{B}}=[\mathrm{g}]_{\mathcal{B}}=\left[\begin{array}{cc}
-\mathbf{1}_{p} & 0 \\
0 & \mathbf{1}_{q}
\end{array}\right], \quad\left[\mathrm{C}_{+}\right]_{\mathcal{B}}=\left[\begin{array}{ll}
S & 0 \\
0 & T
\end{array}\right], \quad\left[\mathrm{C}_{-}\right]_{\mathcal{B}}=\left[\begin{array}{cc}
0 & U \\
-U^{t} & 0
\end{array}\right] .
$$

Here $S$ and $T$ are trace-free symmetric square matrices with components $S^{[u i]}{ }_{[u j]}=-C_{u i u j}=$ $-E_{i j}$ and $T^{[i j]_{[k l]}}=C_{i j k l}$, while $U$ is a $p \times q$ matrix with components $U^{[u i]}{ }_{[j k]}=-C_{u i j k}$. In the PE case, the eigenvalues are the eigenvalues of $S$ and $T$, which are clearly real. In the PM case, we note that the matrix $U$ can be decomposed (using the singular value decomposition) as $U=g_{1} D g_{2}$, where $g_{1}$ and $g_{2}$ are $S O(p)$ and $S O(q)$ matrices, respectively, and $D$ is a diagonal $p \times q$ matrix $D=\operatorname{diag}\left(\lambda_{1}, \lambda_{2}, \ldots, \lambda_{p}\right)$. Consequently, a PM Weyl bivector operator has eigenvalues $\left\{0, \ldots, 0, \pm \mathrm{i} \lambda_{1}, \ldots, \pm \mathrm{i} \lambda_{p}\right\}$, where the number of zero-eigenvalues is at least $N-2 p=q-p=s=\frac{(n-1)(n-4)}{2}$. This proves the proposition.

Remark 3.8. In four dimensions, the original Petrov type classification is equivalent with the Jordan-Segre classification of the Weyl bivector operator (see e.g. [9]), where the latter is diagonalizable iff the Petrov type is I, D or O. Referring to the above we have $p=q=3$, the eigenvalues for $S$ and $T$ are the same (cf section 3.1) and the conditions of the theorem are also sufficient, i.e., if a Weyl operator is diagonalizable and has only real (purely imaginary) eigenvalues then it is PE (PM) w.r.t. a certain $\mathbf{u}$ (see $[9,17]$ and references therein). This can be expressed in terms of polynomial invariants of the self-dual Weyl operator $\mathrm{C}_{s}$ acting on the three-dimensional complex space of self-dual bivectors: defining the quadratic and cubic invariants $I \equiv \operatorname{tr}\left(\mathrm{C}_{s}^{2}\right)$ and $J \equiv \operatorname{tr}\left(\mathrm{C}_{s}^{3}\right)$ and the adimensional invariant $M \equiv I^{3} / J^{2}-6$, the Weyl operator is properly PE (PM) iff it is diagonalizable, $M \in \mathbb{R}^{+} \cup\{\infty\}$ and $I \in \mathbb{R}_{0}^{+}\left(\mathbb{R}_{0}^{-}\right)$. Here $M=0$ corresponds to Petrov type D; the Petrov type I cases were symbolized

7 Notice that the inner product $g$ has now the signature $\frac{(n-1)(n-2)}{2}-(n-1)=\frac{(n-1)(n-4)}{2}, \operatorname{cf}(31)$. However, the map $\theta$ transforms $\left\{\mathbf{u} \wedge \mathbf{m}_{i}, \mathbf{m}_{j} \wedge \mathbf{m}_{k}\right\}$ into $\left\{-\mathbf{u} \wedge \mathbf{m}_{i}, \mathbf{m}_{j} \wedge \mathbf{m}_{k}\right\}$ and the corresponding inner product $\langle-,-\rangle$ on $\wedge^{2} T_{p} M$ is again Euclidean. 
$\mathrm{I}\left(M^{+}\right)$and $\mathrm{I}\left(M^{\infty}\right)$ in the extended Petrov-classification by Arianrhod and McIntosh, where $M=\infty \Leftrightarrow J=0$ corresponds to Petrov type I with a zero eigenvalue [5, 18].

In dimension $n>4$, it is no longer sufficient that the eigenvalues are real (purely imaginary) in order for the Weyl operator to be PE (PM),.even if it is diagonalizable (while it is still necessary, cf proposition 3.7). Counterexamples to the sufficiency for $n=5$ are provided in [19] in the type D case (cf also proposition 3.10 below). However, necessary conditions can be deduced from the fact that, by virtue of proposition 3.7 , the characteristic equations

$$
\sum_{k=0}^{N} a_{k} x^{N-k}=0 \quad\left(a_{0}=1\right)
$$

of the operator $\mathrm{C}$ acting on the full bivector space $\wedge^{2} T_{p} M$ are of the form

$$
\prod_{i=1}^{p}\left(x-\lambda_{i}\right) \prod_{j=1}^{q}\left(x-\mu_{j}\right)=0 \quad \text { or } \quad x^{q-p} \prod_{i=1}^{p}\left(x^{2}+\lambda_{i}^{2}\right)=0,
$$

in the PE or PM case, respectively, where the $\lambda_{i}$ and $\mu_{j}$ are real. Define

$$
A_{k} \equiv \operatorname{tr}\left(\mathrm{C}^{k}\right) \text {. }
$$

- In the PM case, for instance, one has $a_{2 l+1}=0 \Leftrightarrow A_{2 l+1}=0(2 l+1 \leqslant N)$, and $a_{2 l}=0$ for all $l>p(2 l \leqslant N)$, where ( cf e.g. [20] or [21])

$$
a_{2 l}=\frac{(-1)^{l}}{2^{l} l !} \operatorname{det}\left[\begin{array}{lllll}
A_{2} & 2 & 0 & \cdots & 0 \\
A_{4} & A_{2} & 4 & \ddots & \vdots \\
A_{6} & A_{4} & A_{2} & \ddots & 0 \\
\vdots & \ddots & \ddots & \ddots & 2(l-1) \\
A_{2 l} & \cdots & A_{6} & A_{4} & A_{2}
\end{array}\right] \text {. }
$$

In addition $A_{4 l+2}<0<A_{4 l}$ for properly PM.

- In the properly PE case: $A_{2 l}>0$.

In particular, a nilpotent Weyl operator is thus neither PE nor PM. Further necessary conditions on the $A_{k}$ can be derived along the line of [21].

\subsection{Null alignment properties}

In four dimensions, a properly PE or PM Weyl tensor is of Petrov type D or I [5, 22, 23]. This follows immediately from the Weyl-Petrov classification in terms of the operator $Q^{a}{ }_{b}=E^{a}{ }_{b}+\mathrm{i} H^{a}{ }_{b}$ on tangent space (defined w.r.t. any $\mathbf{u}$ ), which has the same Segre type and eigenvalues as the Weyl bivector operator C [9]. Indeed, if a non-zero Weyl tensor is PE (or PM) w.r.t. $\mathbf{u}$, i.e., if the parts $H_{a b}$ (or $E_{a b}$ ) defined w.r.t. this $\mathbf{u}$ vanish, then in any $\mathbf{u}-\mathrm{ONF}$ $\left\{\mathbf{u}, \mathbf{m}_{i=2, \ldots, n}\right\}$ the non-zero part $\left[Q^{i}{ }_{j}\right]$ of the representation matrix is a real symmetric matrix (or a complex unit times such a matrix). Thus $Q_{b}^{a}$, whence $\mathrm{C}$ is diagonalizable and the Petrov type is I or D (cf remark 3.8).

Another classification of the Weyl tensor is the one based on its Debever-Penrose principal null directions (PNDs) which, in four dimensions, coincides with the bivector approach. In higher dimensions, however, both approaches are highly non-equivalent (see [19] for a detailed verification of this in five dimensions). The PNDs approach was worked out in [24] for the Weyl tensor, leading to the concept of Weyl aligned null directions (WANDs) replacing the 
PNDs and being part of the (null) alignment theory for general tensors [25], succinctly revised in section A.3 in the appendix.

In this section we deduce the possible null alignment types for PE/PM Weyl tensors in general $n \geqslant 4$ dimensions, and the uniqueness and relative position to possible (multiple) WANDs of the vectors u realizing the PE/PM property. We do this in a direct way, i.e., without relying on properties of the corresponding Weyl bivector operator.

3.4.1. Admitted alignment types. It immediately follows from propositions 2.4 and 2.7 that a properly PE or PM Weyl tensor is minimal and thus of one of the null alignment types D, I or G, in any dimension $n>4$. However, one can be more specific by giving a different proof.

To this end, the following general observation is essential. Given a unit timelike vector $\mathbf{u}$, a $\mathbf{u}$-adapted null frame is a null frame $\left\{\mathbf{m}_{0}=\boldsymbol{\ell}, \mathbf{m}_{1}=\mathbf{n}, \mathbf{m}_{\hat{i}=3, \ldots, n}\right\}$ for which we have

$$
\mathbf{u}=\frac{\ell-\mathbf{n}}{\sqrt{2}}
$$

In any such frame, the involution (A.8) is represented by

$$
\theta: \ell \leftrightarrow \mathbf{n}, \quad \mathbf{m}_{\hat{i}} \mapsto \mathbf{m}_{\hat{i}}, \quad \forall \hat{i}=3, \ldots, n,
$$

and the (passive) action hereof on a tensor $S$ simply interchanges the frame labels 0 and 1 . This implies that in the case $S=S_{+} \Leftrightarrow \theta(S)=S\left(S=S_{-} \Leftrightarrow \theta(S)=-S\right)$ the components of $S$ in any such frame should be all invariant (change sign). Notice that if a null vector $\ell$ is given, satisfying the normalization condition $l^{a} u_{a}=-1 / \sqrt{2}$ (but it can be otherwise arbitrarily chosen), then (36) should be read as the definition $\mathbf{n}=\ell-\sqrt{2} \mathbf{u}=\theta(\ell)$ of the time-reflected $\ell$, being a null vector lying along the second null direction of the timelike plane $\mathbf{u} \wedge \boldsymbol{\ell}$.

Conversely, if the components of a tensor $S$ in a certain null frame $\left\{\ell, \mathbf{n}, \mathbf{m}_{\hat{i}=3, \ldots, n}\right\}$ are invariant (change sign) under a $0 \leftrightarrow 1$ interchange, then $S=S_{+}(S=S-)$ in the orthogonal splitting w.r.t. the unit timelike vector (36).

Applied to the Weyl tensor we obtain the following.

Proposition 3.9. If a Weyl tensor is $P E / P M$ w.r.t. $\mathbf{u}$ then the following component relations hold in any $\mathbf{u}$-adapted null frame $\left\{\mathbf{m}_{0}=\boldsymbol{\ell}, \mathbf{m}_{1}=\mathbf{n}, \mathbf{m}_{\hat{i}=3, \ldots, n}\right\}$ :

$$
\begin{aligned}
& \text { PE: } \quad C_{0 \hat{i} \hat{0} \hat{j}}=C_{1 \hat{i} 1 \hat{j}}, \quad C_{0 \hat{i} \hat{j} \hat{k}}=C_{1 \hat{i} \hat{j} \hat{k}}, \quad C_{01 \hat{i} \hat{j}}=0 \text {, } \\
& \text { PM: } \quad C_{0 \hat{i} \hat{0} \hat{j}}=-C_{1 \hat{i} \hat{1} \hat{j}}, \quad C_{0 \hat{i} \hat{j} \hat{k}}=-C_{1 \hat{i} \hat{j} \hat{k}}, \quad C_{\hat{i} \hat{j} \hat{k} \hat{l}}=0 .
\end{aligned}
$$

Conversely, if a null frame $\left\{\mathbf{m}_{0}=\boldsymbol{\ell}, \mathbf{m}_{1}=\mathbf{n}, \mathbf{m}_{\hat{i}=3, \ldots, n}\right\}$ exists for which (38), respectively, (39) are satisfied then the Weyl tensor is $P E$, respectively, $P M$ w.r.t. $\mathbf{u}=(\boldsymbol{\ell}-\mathbf{n}) / \sqrt{2}$.

Proof. Due to the properties (A.33) and (A.34), the identities [24]

$$
C_{010 \hat{i}}=C_{0 \hat{k} \hat{i}}^{\hat{k}}, \quad C_{0101}=-\frac{1}{2} C_{\hat{i} \hat{j}}^{\hat{i} \hat{j}}, \quad 2 C_{0(\hat{i} \hat{j}) 1}=C_{\hat{i} \hat{k} \hat{j}}^{\hat{k}}, \quad 2 C_{0[\hat{i} \hat{j}] 1}=-C_{01 \hat{i} \hat{j}}, \quad C_{101 \hat{i}}=C_{1 \hat{k} \hat{i}} \hat{k}^{\hat{k}}
$$

hold in any null frame $\left\{\mathbf{m}_{0}=\boldsymbol{\ell}, \mathbf{m}_{1}=\mathbf{n}, \mathbf{m}_{\hat{i}=3, \ldots, n}\right\}$, and the components of a certain b.w. are fully determined by the following ones:

$$
\text { b.w. 2: } C_{0 \hat{i} \hat{0} \hat{j}}, \quad \text { b.w. 1: } C_{0 \hat{i} \hat{j} \hat{k}}, \quad \text { b.w. 0: } C_{01 \hat{i} \hat{j}}, C_{\hat{i} \hat{j} \hat{k} \hat{l}}, \quad \text { b.w. }-1: C_{1 \hat{i} \hat{j} \hat{k}}, \quad \text { b.w. }-2: C_{1 \hat{i} \hat{j} \hat{j}} \text {. }
$$

The thesis follows from the general considerations above and by observing that under $0 \leftrightarrow 1$ the components $C_{\hat{i} \hat{j} \hat{k} \hat{l}}$ are invariant while $C_{01 \hat{i} \hat{j}}$ change sign.

As a simple consequence we have 
Proposition 3.10. A Weyl tensor which is properly PE or PM w.r.t. a certain $\mathbf{u}$ is of alignment type $D, I_{i}$ or $G$. In the type $I_{i}$ and $D$ cases, the vector $\mathbf{u}$ 'pairs up' the space of WANDs, in the sense that the second null direction of the timelike plane spanned by $\mathbf{u}$ and any WAND is also a WAND with the same multiplicity. Furthermore, a type D Weyl tensor is PE iff it is type $D(d)$, and PM iff it is type $D(a b c)$.

Proof. From (38) and (39) it follows that if in a u-adapted null frame all b.w. +2 components are zero, then also all b.w. -2 components, and similarly for b.w. $+1 /-1$ components; if the b.w. 0 components additionally vanished then the Weyl tensor would be zero (type $\mathrm{O}$ ). Hence, WANDs of a properly PE/PM Weyl tensor (if there exist any) must go in pairs: if $\boldsymbol{\ell}$ spans a WAND then so does $\mathbf{n}$ and with the same multiplicity, which is either 1 (type $\mathrm{I}_{i}$ ) or 2 (type D). This proves the first two statements. The last one follows from these considerations and the definition of type $\mathrm{D}(\mathrm{d})$ and $\mathrm{D}(\mathrm{abc})$ (see section $\mathrm{A} .3$ in the appendix).

Remark 3.11. Proposition 3.9 can be considered as an extension of the observation in $n=4$ dimensions that the Weyl tensor is PE/PM iff in a certain Newman-Penrose null tetrad the relations

$$
\Psi_{0}=c \bar{\Psi}_{4}, \quad \Psi_{1}=-c \bar{\Psi}_{3}, \quad \Psi_{2}=c \bar{\Psi}_{2}
$$

hold, where $c=+1$ in the PE and $c=-1$ in the PM case (see, e.g., [26]). For a Petrov type I Weyl tensor one can always take a Weyl canonical transversal $\left(\Psi_{0}=\Psi_{4} \neq 0, \Psi_{1}=\Psi_{3}=0\right)$ or longitudinal $\left(\Psi_{0}=\Psi_{4}=0, \Psi_{1}=\Psi_{3} \neq 0\right)$ frame and add these to the PE/PM conditions (42). Regarding type $\mathrm{D}$, the last part of proposition 3.10 is an extension of the four-dimensional theorem 4 of [5], stating that a Petrov type D Weyl tensor is PE (PM) iff in a canonical null frame ( $\Psi_{0}=\Psi_{1}=\Psi_{3}=\Psi_{4}=0$ ) the scalar $\Psi_{2} \neq 0$ is real (purely imaginary). Such simplifying choices have been proved crucial for deducing classification or uniqueness results for four-dimensional PE or PM spacetimes (see, e.g., [27]).

Remark 3.12. Spacetimes of type N (such as vacuum type $\mathrm{N}$ pp-waves) are usually understood as describing transverse gravitational waves. The interpretation of type $\mathrm{N}$ fields as 'radiative' is supported, also in higher dimensions, by the peeling behavior of asymptotically flat spacetimes [28] (in spite of significant differences with respect to the four dimensional case, see [28] and references therein). From proposition 3.9 it thus also follows that a spacetime containing gravitational waves necessarily contains both an electric and a magnetic field component. This resembles a well-known similar property of electromagnetic waves, and in four dimensions was discussed, e.g., in [3, 29]. Conversely, we shall show below (section 3.5.1) that static fields (and thus, in particular, the Coulomb-like field of the Schwarzschild solution) are PE.

3.4.2. Uniqueness of $\mathbf{u}$. The following facts are well known in $n=4$ dimensions (see, e.g. $[17,30])$ :

- if a PE/PM Weyl tensor is of Petrov type D, it is PE/PM precisely w.r.t. any $\mathbf{u}$ lying in the plane $\mathcal{L}_{2}$ spanned by the two double WANDs (then also called PNDs [9, 24]);

- if a PE/PM Weyl tensor is of Petrov type I, then it is PE/PM precisely w.r.t. the timelike Weyl principal vector, which is unique up to sign;

- a Weyl tensor can never be properly PE and PM at the same time, even w.r.t. different timelike directions.

We shall see (proposition 3.13) that these properties suitably generalize to any dimension, thus giving further support to the soundness of our PE/PM definitions. Recall that for $n>4$, a 
type D Weyl tensor may have more than two double WANDs (see, e.g., [31-34] for examples). In [35] it is shown that for general $n$, the set of multiple WANDs of a type D Weyl tensor is homeomorphic to a sphere $\mathcal{S}_{k}$, the dimension $k$ being at most $n-4$. This is the sphere of null directions of a (proper) Lorentzian subspace $\mathcal{L}_{k+2}$ (the latter being defined as the space spanned by all multiple WANDs) of the full space $\mathcal{L}_{n}$ (generated by the full sphere of null directions $\mathcal{S}_{n-2}$. However, regarding types $\mathrm{I}_{i}$ and $G$, no analogue of the concept of Weyl principal vector is presently known.

Hence, it is natural to ask whether a PE or PM Weyl tensor of type $\mathrm{I}_{i}$ or $\mathrm{G}$ may admit a non-unique $\mathbf{u}$ when $n>4$. However, we shall show that the answer is negative.

In order to prove our results we will be considering two timelike directions spanned by $\mathbf{u}$ and $\mathbf{u}^{\prime}$, where $u^{a} u_{a}^{\prime}<0, \mathbf{u}^{\prime} \neq \mathbf{u}$. These vectors define two observers in relative motion in the timelike plane $\mathbf{u} \wedge \mathbf{u}^{\prime}$. Suppose that $\boldsymbol{\ell}$ and $\boldsymbol{\ell}^{\prime}$ are two parallel null vectors spanning the first null direction of this plane, while the parallel null vectors $\mathbf{n}$ and $\mathbf{n}^{\prime}$ span the second one, such that

$$
\mathbf{u}=\frac{\ell-\mathbf{n}}{\sqrt{2}}, \quad \mathbf{u}^{\prime}=\frac{\ell^{\prime}-\mathbf{n}^{\prime}}{\sqrt{2}} .
$$

Then $\mathbf{u}^{\prime}=b_{\lambda}(\mathbf{u})$ for a certain positive Lorentz boost (A.24), $\lambda \neq 0$, which transforms a $\mathbf{u}$-adapted null frame $\mathcal{F}=\left\{\mathbf{m}_{0}=\boldsymbol{\ell}, \mathbf{m}_{1}=\mathbf{n}, \mathbf{m}_{\hat{i}=3, \ldots, n}\right\}$ into the $\mathbf{u}^{\prime}$-adapted null frame

$$
\mathcal{F}^{\prime}=b_{\lambda}(\mathcal{F})=\left\{\mathbf{m}_{0^{\prime}}=\ell^{\prime}=\mathrm{e}^{\lambda} \boldsymbol{\ell}, \mathbf{m}_{1^{\prime}}=\mathbf{n}^{\prime}=\mathrm{e}^{-\lambda} \mathbf{n}, \mathbf{m}_{\hat{i}=3, \ldots, n}\right\} .
$$

Proposition 3.13. A Weyl tensor $C$ at a point of a n-dimensional spacetime cannot be properly $P E$ and $P M$ at the same time, even w.r.t. two different timelike directions. If $C$ is properly PE $(P M)$, then it is $P E(P M)$ precisely w.r.t. any $\mathbf{u}$ belonging to the space $\mathcal{L}_{k+2}$ spanned by all multiple WANDs in the type $D$ case, and w.r.t. a unique $\mathbf{u}$ (up to sign) in the type $I_{i}$ and $G$ cases.

Proof. If a Weyl tensor is PE and PM at the same time (w.r.t. two possibly different timelike directions) then the corresponding Weyl operator must be diagonalizable and have zero eigenvalues by proposition 3.7; thus the Weyl tensor is zero, which proves the first statement. Suppose now that $C$ is PE/PM w.r.t. to different timelike directions, spanned by $\mathbf{u}$ and $\mathbf{u}^{\prime}$, where we take $u^{a} u_{a}^{\prime}<0$. Define $\mathbf{u}$ - and $\mathbf{u}^{\prime}$-adapted null frames $\mathcal{F}$ and $\mathcal{F}^{\prime}$ as above. By the $\mathrm{PE} / \mathrm{PM}$ assumptions we have

$C_{0 \hat{i} \hat{0} \hat{j}}= \pm C_{1 \hat{i} \hat{j} \hat{j}}, \quad C_{0 \hat{i} \hat{j} \hat{k}}= \pm C_{1 \hat{i} \hat{j} \hat{k}}, \quad C_{0^{\prime} \hat{i}^{\prime} \hat{j}}= \pm C_{1^{\prime} \hat{i} 1^{\prime} \hat{j}}, \quad C_{0^{\prime} \hat{i} \hat{j} \hat{k}}= \pm C_{1^{\prime} \hat{i} \hat{j} \hat{k}}$.

However, by (44) and the definition of b.w. we also have

$C_{0^{\prime} \hat{i} 0^{\prime} \hat{j}}=\mathrm{e}^{2 \lambda} C_{0 \hat{i} \hat{0} \hat{j}}, \quad C_{1^{\prime} \hat{i}^{\prime} \hat{j}}=\mathrm{e}^{-2 \lambda} C_{1 \hat{i} 1 \hat{j}}, \quad C_{0^{\prime} \hat{i} \hat{j} \hat{k}}=\mathrm{e}^{\lambda} C_{0 \hat{i} \hat{j} \hat{k}}, \quad C_{1^{\prime} \hat{i} \hat{j} \hat{k}}=\mathrm{e}^{-\lambda} C_{1 \hat{i} \hat{j} \hat{k} k}$,

By comparison of (45) and (46) and the fact that $e^{\lambda} \neq 1$ we immediately obtain

$$
C_{0 \hat{i} 0 \hat{j}}=0=C_{1 \hat{i} 1 \hat{j}}, \quad C_{0 \hat{i} \hat{j} \hat{k}}=0=C_{1 \hat{i} \hat{j} \hat{k}},
$$

i.e., the type $\mathrm{D}$ condition is fulfilled relative to $\boldsymbol{\ell}$ and $\mathbf{n}$, which thus span double WANDs. This already proves uniqueness of the $\mathbf{u}$-direction in the type $\mathrm{I}_{i}$ and $G$ cases.

Next, suppose that $C$ is of type D and PE/PM w.r.t. u. By the second sentence in proposition 3.10, such a $\mathbf{u}$ necessarily lies in a plane of double WANDs and thus in $\mathcal{L}_{k+2}$. Conversely, consider any other timelike direction in $\mathcal{L}_{k+2}$, spanned by a vector $\mathbf{u}^{\prime}$ $\left(u^{a} u_{a}^{\prime}<0, \mathbf{u}^{\prime} \neq \mathbf{u}\right)$. Then, by definition of the vector space $\mathcal{L}_{k+2}$, the null directions of the timelike plane $\mathbf{u} \wedge \mathbf{u}^{\prime}$ are double WANDs. Hence, defining again $\mathbf{u}$ - and $\mathbf{u}^{\prime}$-adapted null frames $\mathcal{F}$ and $\mathcal{F}^{\prime}$ as above, the only non-zero Weyl components in the $\mathcal{F}$-frame are comprised in the b.w. 0 components which are invariant or change sign under (A.8), namely $C_{\hat{i} \hat{j} \hat{k} \hat{l}}$ (PE case) or $C_{01 \hat{i} \hat{j}}$ (PM case). Since the boost $b_{\lambda}$ leaves these components invariant (by definition of b.w.), the same holds in the $\mathcal{F}^{\prime}$-frame, and thus the Weyl tensor is also PE/PM also w.r.t. $\mathbf{u}^{\prime}$. 
Remark 3.14. The proof of this proposition can be readily generalized to arbitrary tensors $S$. We notice that if $S$ is of type D (cf section 2.3) then the set of null directions along which the boost order of $S$ is zero is again homeomorphic to a sphere $\mathcal{S}_{k}$ generating a Lorentzian space $\mathcal{L}_{k+2}$ [36]. We obtain that any tensor $S \neq 0$ cannot be $S_{+}$and $S_{-}$at the same time, even w.r.t. two different timelike directions. If $S=S_{ \pm}$w.r.t. a certain $\mathbf{u}$, then either $S$ is not of type II or more special, in which case $S=S_{ \pm}$is realized by a unique timelike direction, or $S$ is of type $D$, in which case $S=S_{ \pm}$is realized by any $\mathbf{u} \in \mathcal{L}_{k+2}$.

Remark 3.15. More specifically for a type D Weyl tensor $C$, it also follows from the results of [35] that if $C$ has more than two double WANDs (i.e., we have $k \geqslant 1$ for the dimension of $\mathcal{S}_{k}$ ), then $C=C_{+}$w.r.t. any $\mathbf{u}$ lying in $\mathcal{L}_{k+2}$, i.e., $C$ is $P E$ (type $D(d)$ ). Let us emphasize once more that in this case the PE property is realized precisely by any $\mathbf{u} \in \mathcal{L}_{k+2}$ (i.e., by any $\mathbf{u}$ lying in any plane spanned by multiple WANDs, and by no other timelike vectors); hence, since $k \leqslant n-3$ for any $n$ [35], a Weyl tensor can never be PE w.r.t. all timelike directions in $\mathcal{L}_{n}$. By contraposition, we have that a type $\mathrm{D}$ spacetime that is not $\mathrm{PE}$ admits exactly two multiple WANDs. This is true, in particular, for a type D PM Weyl tensor, which is thus PM w.r.t. all timelike directions in the 2-plane $\boldsymbol{\ell} \wedge \mathbf{n}$, and only w.r.t. those (i.e., $k=0$ for PM Weyl in proposition 3.13).

Remark 3.16. For PE or PM type $\mathrm{I}_{i}$ Weyl tensors, the second statement of proposition 3.10 becomes particularly meaningful when combined with the u-uniqueness result: any single WAND is associated to exactly one other single WAND under the uniquely defined timereflection $\theta$, the relation being symmetric and where (36) should be read as $\sqrt{2} \mathbf{u}=\boldsymbol{\ell}-\theta(\boldsymbol{\ell})=$ $\theta(\theta(\ell))-\theta(\ell)$. This is exemplified clearly, e.g., by the four single WANDs of static black rings [37] (which are PE, see below). In $n=4$ dimensions a Petrov type I spacetime has always four PNDs, and it was known that these span a three-dimensional vector space in the $\mathrm{PE}$ and PM cases [22, 38, 5]; this is now a simple consequence of the 'pairing' property (second statement of proposition 3.10).

\subsection{PE spacetimes}

Large classes of PE spacetimes exist. It is not our purpose to deduce classifications of, for instance, PE Einstein spacetimes here; even in four dimensions this is a very difficult task which is still far from completion. Instead, we mention generic conditions which imply that the spacetimes in question are PE w.r.t. some $\mathbf{u}$. These generic conditions hold in arbitrary dimensions and often generalize known ones in four dimensions. Hence, this again supports the soundness of the Weyl PE definition, cf section 3.4.2. Evidently, all examples remain PE, with the same Weyl alignment type, when subjected to a conformal transformation (this will be important in section 4).

3.5.1. Spacetimes with a shear-free normal $\mathbf{u}$, static metrics and warps with a one-dimensional timelike factor. Given a unit timelike vector field $\mathbf{u}$, we refer to (C.1)-(C.5) of appendix C for the usual definitions of the kinematic quantities of $\mathbf{u}$. In particular, a vector field $\mathbf{u}$ and the timelike congruence of curves it generates, are called shear-free if $\sigma_{a b}=0$, and normal (or non-rotating or twist-free or hypersurface-orthogonal) if $\omega_{a b}=0$. We have

Proposition 3.17. All spacetimes admitting a shear-free, normal unit timelike vector field $\mathbf{u}$ are PE w.r.t. $\mathbf{u}$. These are precisely the spacetimes which admit a line element of the form

$$
\mathrm{d} s^{2}=-V^{2}\left(t, x^{\gamma}\right) \mathrm{d} t^{2}+P^{2}\left(t, x^{\gamma}\right) \xi_{\alpha \beta}\left(x^{\gamma}\right) \mathrm{d} x^{\alpha} \mathrm{d} x^{\beta} .
$$


In these coordinates we have $\mathbf{u}=\partial_{t} / V$, and the remaining kinematic quantities are given by

$$
\tilde{\Theta}=\frac{1}{V} \partial_{t} \ln P, \quad \dot{u}_{\alpha}=\partial_{\alpha} \ln V
$$

Proof. Equation (C.12) gives the magnetic part of the Weyl tensor in terms of the kinematic quantities. As an immediate consequence, the existence of $\mathbf{u}$ for which $\sigma_{a b}=\omega_{a b}=0$ implies that the magnetic part vanishes and the spacetime is PE w.r.t. $\mathbf{u}$. Since $\mathbf{u}$ is hypersurface-orthogonal one has $u_{a}=-V\left(t, x^{\gamma}\right) \mathrm{d}_{a} t$ and the line element can be written as $\mathrm{d} s^{2}=-V\left(t, x^{\gamma}\right)^{2} \mathrm{~d} t^{2}+h_{\alpha \beta}\left(t, x^{\gamma}\right) \mathrm{d} x^{\alpha} \mathrm{d} x^{\beta}$, for certain coordinates $\left\{t, x^{\gamma}\right\}$. Then the shearfree property of $u^{a}$ translates to $\tilde{\Theta} h_{\alpha \beta}=u_{\alpha ; \beta}=\frac{1}{2 V} \partial_{t} h_{\alpha \beta}$ (the labels referring to coordinate components here), whence $h_{\alpha \beta}=P\left(t, x^{\gamma}\right)^{2} \xi_{\alpha \beta}\left(x^{\gamma}\right)$ (and vice versa; this is a direct extension of the observations in [39] from four to arbitrary dimensions). The expressions (49) follow by direct computation.

Remark 3.18. One may ask the converse question: does every PE spacetime necessarily admit one or more shear-free normal timelike congruences? In conformally flat (type O) spacetimes the answer is yes: there are as many of them as in Minkowski spacetime, since the conditions $\sigma_{a b}=0, \omega_{a b}=0$ are conformally invariant (see, e.g., [9]). In four dimensions, partial answers are known for the other admitted Petrov types (D and I). In the Petrov type D case, it was shown in [40] that in PE Einstein spacetimes and aligned Einstein-Maxwell solutions there is a one-degree freedom of shear-free normal timelike congruences. Well-known examples of PE type D Einstein spacetimes are the Schwarzschild and C metric solutions (see [40] for a complete survey). For instance, in the interior (non-static) region $u(r) \equiv 2 m / r-1>0$ of the Schwarzschild solution $\mathrm{d} s^{2}=-\mathrm{d} r^{2} / u(r)+u(r) \mathrm{d} t^{2}+r^{2}\left(\mathrm{~d} \theta^{2}+\sin (\theta)^{2} \mathrm{~d} \phi^{2}\right)$, two particular families of shear-free normal vector fields $\mathbf{u}$ are given by

$\mathbf{u}=\sqrt{E} r \partial_{r}+(2 m)^{1 / 3}\left(\frac{1}{\sqrt{u(r) q(r)}}-\frac{\sqrt{E} r}{u(r)}\right) \partial_{t}, \quad q(r)=\frac{E r^{2}}{u(r)}-1 \pm \sqrt{\left(\frac{E r^{2}}{u(r)}-1\right)^{2}-1}$,

where, for a given $r$, the constant $E>0$ is large enough such that $E r^{2}>u(r)$; shear-free normal congruences also exist in the exterior regions, where they generalize the static observers. In passing, we note that all Petrov type D perfect fluids with shear-free normal fluid velocity, comprising the type D PE Einstein spacetimes as a limiting subcase, were classified by Barnes [41] (see also [40] for a clarification and a correction). However, the answer to the question is negative in general. For instance, Gödel's rotating perfect fluid universe and the Szekeres non-rotating dust models (see [9] and references therein), both of type D, are PE but do not admit a shear-free normal $\mathbf{u}$ (since the conditions of proposition B.1 in [40] are not fulfilled). In the Petrov type I case the same is true for, e.g., the generic Kasner vacuum spacetimes and the rotating 'silent' dust models of [42]; here the field $\mathbf{u}$ realizing the PE condition is unique (proposition 3.13) and one verifies that it is not shear-free normal, while proposition 3.17 ensures that there cannot be any other shear-free normal timelike congruences.

Special cases of the spacetimes (48) are the following warped (cases (a) and (b) below), direct product (case (c)) and doubly-warped (case (d)) spacetimes with a one-dimensional timelike factor (see also [43]; we add the expressions of the corresponding expansion scalar and acceleration vector between square brackets, a prime denoting an ordinary derivative):

(a) $V=V(t), P=P(t)\left[\tilde{\Theta}=P^{\prime}(t) /(P(t) V(t)), \dot{u}_{a}=0\right]$;

(b) $V=V\left(x^{\gamma}\right), P=P\left(x^{\gamma}\right)\left[\tilde{\Theta}=0, \dot{u}_{a}=\ln (V)_{; a}\right]$; 
(c) $V=V(t), P=P\left(x^{\gamma}\right)\left[\tilde{\Theta}=0, \dot{u}_{a}=0\right]$;

(d) $V=V\left(x^{\gamma}\right)$ non-constant, $P=P(t)$ non-constant $\left[\tilde{\Theta}=P^{\prime}(t) /\left(P(t) V\left(x^{\gamma}\right)\right)\right.$, $\dot{u}_{a}=$ $\left.\ln (V)_{; a}\right]$.

Notice that if $V=V(t)$, we may rescale the coordinate $t$ such that $V=1$; if $P=P\left(x^{\gamma}\right)$ we can put $P=1$ by absorption in $\xi_{\alpha \beta}\left(x^{\gamma}\right)$. Hence, the direct product case (c) can be considered as a subcase of both (a) and (b). Case (d) describes doubly-warped spacetimes; see [44] for a definition and for a discussion of their properties in four dimensions.

It is easy to see (cf appendix A of [34] and references therein) that for Einstein spacetimes case (a) reduces to Brinkmann's warp ansatz [45]

$$
\mathrm{d} s^{2}=-f(t)^{-1} \mathrm{~d} t^{2}+f(t) \mathrm{d} \tilde{s}^{2}, \quad f(t)=\lambda t^{2}-2 \mathrm{~d} t-b,
$$

where $\lambda$ is the cosmological constant (up to a positive numerical factor), $b$ and $d$ are constant parameters and $\mathrm{d} \tilde{s}^{2}$ is any $(n-1)$-dimensional Euclidean Einstein space with Ricci scalar $\tilde{\mathcal{R}}=-(n-1)(n-2)\left(\lambda b+d^{2}\right)$. This can be used to produce a number of explicit examples (see [34] for a recent analysis of such warps).

Case (b) precisely covers the static spacetimes (u being parallel to the hypersurfaceorthogonal timelike Killing vector field $\partial_{t}$ ). In fact, the argument in the proof of proposition 3.10 is a simple extension of the one used in [43] to prove that static spacetimes can only be of the Weyl types $\mathrm{O}, \mathrm{D}(\mathrm{d}), \mathrm{I}_{i}$ or $\mathrm{G}$. Let us note that in $n>4$ dimensions explicit static vacuum solutions of the last three types are known (type O just giving flat space): the Schwarzschild black hole (type D [24, 43, 46]), the static black ring (type $\mathrm{I}_{i}[37]$ ) and the static KK bubble (type $\mathrm{G}$ [31]). In four dimensions, the static type $\mathrm{D}$ vacua were invariantly classified by Ehlers and Kundt [47] and comprise, e.g., the exterior regions of the Schwarzschild and $C$ metrics; static type I examples are comprised in, e.g., the Harrison metrics (see [9]).

Remark 3.19. In four dimensions, and in the line of remark 3.18, the following spacetimes are necessarily static (u being parallel to the hypersurface-orthogonal timelike Killing vector field $\partial_{t}$ ):

- Petrov type D Einstein spacetimes with a non-rotating rigid $\mathbf{u}$ (i.e., $\omega_{a b}=0, \sigma_{a b}=0=$ $\tilde{\Theta}=0)[40]$;

- Petrov type I Einstein spacetimes with a shear-free normal u [22, 39], and type I perfect fluids with shear-free normal fluid velocity $\mathbf{u}$ [41].

Remark 3.20. Stationary spacetimes. Although stationary PE spacetimes do exist, and in fourdimensions have been constructed in [48-51], this is now not the only possibility (contrary to the static case discussed above). First, the existence of four-dimensional Petrov type I, stationary spacetimes with a PM Weyl tensor was shown in [52]. Moreover, stationary, nonstatic spacetimes are in general 'hermaphroditic', i.e., neither PE nor PM. For instance, in four dimensions generic (Petrov type D) locally rotationally symmetric (LRS) spacetimes of class I in the Stewart-Ellis classification [53] have this property (cf [54] for the additional PE and PM conditions). The same is true for the exterior (Petrov type I or II) vacuum region of a van Stockum rotating dust cylinder (if the mass per unit length is large enough), and the (Petrov type D) Kerr metric [55]. We additionally point out here that the higher-dimensional generalization of the latter, i.e. the black hole solution of Myers and Perry [56], shares a similar property: the components of the type D Weyl tensor in a canonical null frame are such that $C_{i j k l} \neq 0 \neq C_{01 i j}$ (see section 6.4 of [43] and section 5.5 of [57]), such that the spacetime is neither PE (type $\mathrm{D}(\mathrm{d})$ ) nor PM (type $\mathrm{D}(\mathrm{abc})$ ), cf proposition 3.10 (more generally, the same comment applies to all vacuum Kerr-Schild spacetimes with a twisting Kerr-Schild null vector, see section 5.5.1 of [57]). Moreover, the (generically type $\mathrm{I}_{i}$ ) five-dimensional spinning 
black rings of [58] (reducing to a Myers-Perry black hole under an appropriate limit) are also hermaphroditic in the non-static regions, as can be shown by making use of the Bel-Debever criteria of proposition 3.5. These thus provide explicit examples of spacetimes with a minimal Weyl tensor (cf proposition 2.7) which are, however, neither PE nor PM.

3.5.2. More general direct products and warped spacetimes. We have seen above that warped metrics with a one-dimensional timelike factor are examples of PE spacetimes and thus can only be of type $\mathrm{G}, \mathrm{I}_{i}, \mathrm{D}(\mathrm{d})$ or $\mathrm{O}$ (proposition 3.10). This latter result was stated in proposition 3 of [43]. Here we discuss similar properties in the case of other possible warps $(M, g)$ for which, by definition:

- $M$ is a direct product manifold $M^{(n)}=M^{\left(n_{1}\right)} \times M^{\left(n_{2}\right)}$ of factor spaces $M^{\left(n_{1}\right)}$ and $M^{\left(n_{2}\right)}$, where $n=n_{1}+n_{2}, n_{1} \geqslant 2$ and $M^{\left(n_{1}\right)}$ represents the Lorentzian (timelike) factor;

- $\boldsymbol{g}$ is conformal to a direct sum metric,

$$
\boldsymbol{g}=\mathrm{e}^{2 \theta}\left(\boldsymbol{g}^{\left(n_{1}\right)} \oplus \boldsymbol{g}^{\left(n_{2}\right)}\right),
$$

where $\boldsymbol{g}^{\left(n_{i}\right)}$ is a metric on $M^{\left(n_{i}\right)}(i=1,2)$ and $\theta$ is a smooth scalar function on either $M^{\left(n_{1}\right)}$ or $M^{\left(n_{2}\right)}$ (see, e.g., [59]).

Since we will be interested in PE/PM Weyl tensors of direct products, it is useful first of all to recall a known result (see, e.g., [43]) that tells us when the Weyl tensor of a product metric vanishes (and is thus both, trivially, PE and PM): a product space is conformally flat iff both product spaces are of constant curvature and

$$
n_{2}\left(n_{2}-1\right) \mathcal{R}^{\left(n_{1}\right)}+n_{1}\left(n_{1}-1\right) \mathcal{R}^{\left(n_{2}\right)}=0 .
$$

In the following analysis we shall mostly rely on the results of [43]. First, combining propositions 4 and 5 (and the explanation on top of page 4415 of [43]) with our proposition 3.13 we obtain

Proposition 3.21. Warped spacetimes with a two-dimensional Lorentzian factor $\left(M^{\left(n_{1}\right)}, \boldsymbol{g}^{\left(n_{1}\right)}\right)$, $n_{1}=2$, are at each point either type $O$, or type $D$ and $P E$ w.r.t. any unit timelike vector living in $M^{\left(n_{1}\right)}$, the uplifts of the null directions of the tangent space to $\left(M^{\left(n_{1}\right)}, \boldsymbol{g}^{\left(n_{1}\right)}\right)$ being double WANDs of the complete spacetime $(M, g)$. They include, in particular, all spherically, hyperbolically or plane symmetric spacetimes.

Here and below, a vector is said to 'live' in a factor space if it is spanned by uplifts of tangent vectors to this space. For warped products in which the Lorentzian factor is at least three-dimensional the above proposition does not hold, in general. However we can find necessary and sufficient conditions for the product space to be PE. Let us give results in the case of direct products $(\theta=0$ in (52)). This can be then extended to warped products (in fact, to all conformally related spaces) by introducing a suitable conformal factor, which does not affect the properties of the Weyl tensor. For direct products there is a biunivocal relation between vectors $\mathbf{v}$ tangent to $M^{\left(n_{i}\right)}$ and their uplifts $\mathbf{v}^{*}$ living in $M^{\left(n_{i}\right)}$ ( $\mathbf{v}$ being the $M^{\left(n_{i}\right)}$-projection of $\left.\mathbf{v}^{*}\right)$. For brevity, we shall identify these objects and use the same notation for them; it will be clear from the context to what quantity we are referring. Also, we let lowercase Latin letters serve as abstract indices for the full space as well as for the factor spaces. We denote by $R_{a b}^{\left(n_{i}\right)}$ the Ricci tensor of $M^{\left(n_{i}\right)}$, and similarly for other tensors defined in the factor geometries. In addition, given a unit timelike $\mathbf{U}$ tangent to $M^{\left(n_{1}\right)}$ we define a $\mathbf{U}-\mathrm{ONF}$ $\left\{\mathbf{U}, \mathbf{m}_{A}\right\}$ (with frame labels $\left.A, B, C, \ldots=2, \ldots, n_{1}\right)$ of $M^{\left(n_{1}\right)}$ and an ONF $\left\{\mathbf{m}_{I}\right\}$ (with frame labels $\left.I, J, K, \ldots=n_{1}+1, \ldots, n\right)$ of $M^{\left(n_{2}\right)}$. These in turn enable us to define a composite $\mathbf{U}-\mathrm{ONF}\left\{\mathbf{U}, \mathbf{m}_{i=2, \ldots, n}\right\}$ of $M^{(n)}$. Then, using the results of section 4 of [43] we easily arrive at 
Proposition 3.22. A direct product spacetime $M^{(n)}=M^{\left(n_{1}\right)} \times M^{\left(n_{2}\right)}$ is PE w.r.t. a unit timelike vector $\mathbf{U}$ that lives in $M^{\left(n_{1}\right)}$ iff $\mathbf{U}$ is an eigenvector of $R_{a b}^{\left(n_{1}\right)}$ and $M^{\left(n_{1}\right)}$ is PE w.r.t. $\mathbf{U}$, i.e.,

$$
R_{U A}^{\left(n_{1}\right)}=0, \quad C_{U A B C}^{\left(n_{1}\right)}=0 .
$$

Then, $\mathbf{U}$ is also an eigenvector of the Ricci tensor $R_{a b}$ of $M^{(n)}$ (i.e., $\left.R_{U i}=0\right)$.

Proof. By (9) and (10) of [43], the requirements $C_{U I A J}=0$ and $C_{U A B C}=0$ are equivalent to (54), while the remaining magnetic Weyl components of $M^{(n)}$ are always identically zero thanks to equation (8) of [43]. This proves the first part. The second part follows from the well-known fact that the Ricci tensor of a direct product is a 'product tensor' (i.e., it is decomposable), such that $R_{U A}=R_{U A}^{\left(n_{1}\right)}=0$ and $R_{U I}=0$.

Remark 3.23. The proof makes use of equation (9) of [43], which is only valid for $n_{1} \geqslant 3$. However, the proposition remains true for $n_{1}=1$ or $n_{1}=2$, since then the spacetime is always PE (cf above) and the conditions (54) are identically satisfied indeed. Further notice that in the case $n_{1}=3$ the Weyl tensor of $M^{\left(n_{1}\right)}$ is identically zero, such that $M^{(n)}$ is PE w.r.t. $\mathbf{U}$ iff the Ricci tensor of $M^{\left(n_{1}\right)}$ has $\mathbf{U}$ as an eigenvector. In general, we shall be able to rephrase this proposition once we have introduced the concept of Riemann PE spacetime in the next section.

One may further wonder whether direct products exist which are PE w.r.t. a vector $\mathbf{u}$ not living in $M^{\left(n_{1}\right)}$, i.e., being inherently $n$-dimensional. Since the $M^{\left(n_{2}\right)}$-projection of $\mathbf{u}$ is spacelike, the $M^{\left(n_{1}\right)}$-projection is timelike. Thus we have $\mathbf{u}=\cosh \gamma \mathbf{U}+\sinh \gamma \mathbf{Y}$, where $\mathbf{U}$ is a unit timelike vector living in $M^{\left(n_{1}\right)}, \mathbf{Y}$ a unit spacelike vector living in $M^{\left(n_{2}\right)}$ and $\gamma \neq 0$. We also define the unit spacelike vector $\mathbf{y}=\cosh \gamma \mathbf{Y}+\sinh \gamma \mathbf{U}$ and use a further adapted $\mathbf{u}-\mathrm{ONF}$ $\left\{\mathbf{u}, \mathbf{m}_{i=2, \ldots, n}\right\}=\left\{\mathbf{u}, \mathbf{m}_{A}, \mathbf{y}, \mathbf{m}_{\tilde{I}}\right\}$, where the $\left(n_{1}-1\right) \mathbf{m}_{A}$ live in $M^{\left(n_{1}\right)}$ and the $\left(n_{2}-1\right) \mathbf{m}_{\tilde{I}}$ in $M^{\left(n_{2}\right)}$.

Proposition 3.24. A direct product spacetime $M^{(n)}=M^{\left(n_{1}\right)} \times M^{\left(n_{2}\right)}$ is PE w.r.t. a unit timelike vector $\mathbf{u}=\cosh \gamma \mathbf{U}+\sinh \gamma \mathbf{Y}$ not living in $M^{\left(n_{1}\right)}\left(\gamma \neq 0\right.$, $\mathbf{U}$ living in $M^{\left(n_{1}\right)}$ and $\mathbf{Y}$ in $\left.M^{\left(n_{2}\right)}\right)$ iff the following relations hold:

$$
\begin{array}{lcl}
C_{U A B C}^{\left(n_{1}\right)}=0, & R_{U A}^{\left(n_{1}\right)}=0, & \left(n_{1}-1\right) R_{U A U B}^{\left(n_{1}\right)}=R_{U U}^{\left(n_{1}\right)} \delta_{A B} \\
C_{Y \tilde{I} \tilde{I} \tilde{K}}^{\left(n_{2}\right)}=0, & R_{Y \tilde{I}}^{\left(n_{2}\right)}=0, \quad\left(n_{2}-1\right) R_{Y \tilde{I} Y \tilde{J}}^{\left(n_{2}\right)}=R_{Y Y}^{\left(n_{2}\right)} \delta_{\tilde{I} \tilde{J}} \\
\left(n_{2}-1\right) R_{U U}^{\left(n_{1}\right)}=\left(n_{1}-1\right) R_{Y Y}^{\left(n_{2}\right)} . &
\end{array}
$$

In particular, $M^{(n)}$ is PE w.r.t. $\mathbf{U}$ and thus belongs to the class described by proposition 3.22 . Moreover, it is either type $O$, or type $D$ and $P E$ w.r.t. any $\mathbf{u}$ in the plane spanned by $\mathbf{U}$ and $\mathbf{Y}$, i.e., w.r.t. $\mathbf{u}=\cosh \gamma \mathbf{U}+\sinh \gamma \mathbf{Y}$ for any $\gamma$.

Proof. The proof goes by splitting the equations $C_{u i j k}=0$ in the adapted frame $\left\{\mathbf{u}, \mathbf{m}_{i=2, \ldots, n}\right\}=$ $\left\{\mathbf{u}, \mathbf{m}_{A}, \mathbf{y}, \mathbf{m}_{\tilde{I}}\right\}$ and employing equations (8)-(11) of [43]. Requiring $C_{u \tilde{I} A \tilde{J}}=0$ and $C_{u A B C}=0$ one finds (54) so that, by proposition $3.22, M^{(n)}$ is PE also w.r.t. to the timelike unit vector field $\mathbf{U}$ living in $M^{\left(n_{1}\right)}$. Direct products which are PE w.r.t. a vector $\mathbf{u}$ not living in $M^{\left(n_{1}\right)}$ are thus a subset of those considered in proposition 3.22. Since they are PE w.r.t. two distinct timelike vector fields, by proposition 3.13 they are necessarily of type D (unless conformally flat) and thus PE w.r.t. any unit timelike vector in the plane spanned by $\mathbf{u}$ and $\mathbf{U}$ (cf proposition 3.13). Dually, $C_{u A \tilde{I} B}=0$ and $C_{u \tilde{I} \tilde{I} \tilde{K}}=0$ are equivalent to the first two relations in (56). Finally, $C_{u A y B}=0$ and $C_{u \tilde{I} y \tilde{J}}=0$ give $C_{U A U B}+C_{Y A Y B}=0$ and $C_{U \tilde{I} U \tilde{J}}+C_{Y \tilde{I} Y \tilde{J}}=0$, respectively. Tracing 
the first relation over $A$ and $B$ (or the second over $\tilde{I}$ and $\tilde{J}$ ) yields (57), and then the respective relations reduce to the last equations of (55) and (56). Under (55)-(57) the remaining Weyl magnetic components turn out to be identically zero. This proves the proposition.

Simple examples are given by spacetimes $M^{(n)}=M^{\left(n_{1}\right)} \times M^{\left(n_{2}\right)}$ with metric $\mathrm{d} s^{2}=$ $\mathrm{d} s_{1}^{2}+\mathrm{d} s_{2}^{2}$, with

$\mathrm{d} s_{1}^{2}=-\mathrm{d} t^{2}+\mathrm{d} \Xi^{2}, \quad \mathrm{~d} s_{2}^{2}=\mathrm{d} z^{2}+\mathrm{d} \Sigma^{2}, \quad \mathrm{~d} \Xi^{2}$ and $\mathrm{d} \Sigma^{2}$ Ricci-flat Euclidean spaces.

Here $\mathbf{U}=\partial_{t}$ and $\mathbf{Y}=\partial_{z}$, and the full space as well as the factors are Ricci flat with decomposable Weyl tensor ${ }^{8}$.

3.5.3. Spacetimes with certain isotropies. A spacetime with a high degree of symmetry clearly has a special Weyl tensor. In particular, an isotropy of spacetime imposes constraints on the Weyl tensor in the sense that the isotropy must leave the Weyl tensor invariant; consequently, a non-trivial isotropy implies that certain components of the Weyl tensor are zero. Recall that the isotropy group of an $n$-dimensional spacetime must be isomorphic to a subgroup of the Lorentz group $S O(1, n-1)$. The largest possible isotropy group is thus of dimension $n(n-1) / 2$, in which case the spacetime must be of constant curvature, and therefore also conformally flat (see, e.g., [9]). However, some (weaker) restrictions also arise in the presence of a smaller isotropy. In the context of PE spacetimes, an interesting result is the following:

Proposition 3.25. A spacetime which admits $S O\left(p_{1}\right) \times \cdots \times S O\left(p_{i}\right) \times \cdots \times S O\left(p_{k}\right)$ isotropy, where $p_{i} \geqslant 2$ and $\sum_{i=1}^{k} p_{i}=n-1$, is $P E$.

Proof. Consider the ONF adapted to the isotropy group as follows: the factor $S O\left(p_{i}\right)$ acts on (and leaves invariant) the $p_{i}$-plane spanned by $\mathbf{m}^{a_{i}}$. Let $\left(h_{i}\right)_{b}^{a}$ be the corresponding projection operators onto this $p_{i}$-plane and define the spatial projector $h_{b}^{a}=\sum_{i=1}^{k}\left(h_{i}\right)_{b}^{a}$. The action of the isotropy group can thus be put on a block-diagonal form; explicitly, for $G=\left(G_{l}, \ldots, G_{i}, \ldots, G_{k}\right) \in S O\left(p_{1}\right) \times \cdots \times S O\left(p_{i}\right) \times \cdots \times S O\left(p_{k}\right)$, the isotropy acts on a vector $v$ as:

$$
G(v)=\sum_{i=1}^{k} G_{i} h_{i}(v) .
$$

Since $\sum p_{i}=n-1$ there will be a timelike vector $\mathbf{u}$ so that $g_{a b}=-u_{a} u_{b}+h_{a b}$.

Consider then the tensor $T_{e f g} \equiv u^{a} C_{a b c d} h_{e}^{b} h_{f}^{c} h_{g}^{d}$. This is a purely spatial tensor relative to $\mathbf{u}$, with components $T_{i j k}=C_{u i j k}$ in any $\mathbf{u}-\mathrm{ONF}$, such that it is necessary and sufficient to show that $T_{\text {efg }}=0$. The Weyl tensor is invariant under the spacetime isotropy group, and using the results regarding invariant tensors under the action of $S O(p)$ groups (see [61]), the only purely spatial tensors invariant under the group in question are linear combinations of tensor products of $\left(h_{i}\right)_{a b}$ and the totally antisymmetric $p_{i}$-tensors $\boldsymbol{\epsilon}^{i}=\bigwedge_{a_{i}} \mathbf{m}^{a_{i}}$. Since the tensor $T$ is a rank 3 tensor, it follows that $T$ must be of the form $T=\sum_{i=1}^{k} \alpha_{i} \epsilon^{i}, \alpha_{i} \in \mathcal{F}_{M}$. Hence, $T_{\text {efg }}=T_{[e f g]}$. However, due to the first Bianchi identity (last equation in (A.33)) we have $u^{a} C_{a[b c d]}=0$, whence $T_{[e f g]}=0$, which proves the proposition.

Special instances of the above isotropy are the following.

8 Recall (see $[60,43]$ ) that a direct product space is an Einstein space iff both factors are Einstein spaces and $\mathcal{R} / n=\mathcal{R}^{\left(n_{1}\right)} / n_{1}=\mathcal{R}^{\left(n_{2}\right)} / n_{2}$; it has decomposable Weyl tensor iff both factors are Einstein spaces and $n_{2}\left(n_{2}-1\right) \mathcal{R}^{\left(n_{1}\right)}+n_{1}\left(n_{1}-1\right) \mathcal{R}^{\left(n_{2}\right)}=0$. Hence, a direct product is Ricci flat iff both factor spaces are Ricci-flat (in which case the Weyl tensor is automatically decomposable). This applies to the factors and thus to the full space (58). 
- Spacetimes with an isotropy group $S O(n-1)$. They are conformally flat (see, e.g., theorem 7.1 of [16]), i.e., proposition 3.25 becomes 'trivial' if we take one single $S O(n-1)$ factor. If the spacetime is not of constant curvature, the $S O(n-1)$ isotropy and the conformal flatness imply that the Ricci tensor has Segre type $\{1,(11 \cdots 1)\}$. Also, the shear, rotation and acceleration of the preferred vector field $\mathbf{u}$ must vanish, while the surfaces of the foliation orthogonal to $\mathbf{u}$ are maximally isotropic and thus have constant curvature [9]. It follows that the spacetimes with an isotropy group $S O(n-1)$ are given by the line elements

$$
\mathrm{d} s^{2}=-\mathrm{d} t^{2}+a(t)^{2} \mathrm{~d} \Omega_{n-1, k}^{2}\left(x^{1}, \ldots, x^{n-1}\right),
$$

where $\mathrm{d} \Omega_{n-1, k}^{2}$ is the metric on an $(n-1)$-dimensional 'unit' space of constant curvature with sign $k$. Notice that they are special instances of the warped metrics (48), case (a). For $n=4$ this gives the Friedmann-Lemaitre Robertson-Walker (FLRW) model, which is in fact the only possibility to satisfy the isotropy condition of proposition 3.25. However, in all higher dimensions spacetimes satisfying the assumptions of proposition 3.25 and admitting non-zero Weyl tensors are possible (as is generically the case in the next examples).

- In even dimensions, a possible isotropy is $S O(3) \times S O(2)^{(n-4) / 2}$. This is admitted, for example, by the metric

$$
\mathrm{d} s^{2}=-\mathrm{d} t^{2}+a(t)^{2} \mathrm{~d} \Omega_{3, k}^{2}(x, y, z)+\sum_{i=1}^{(n-4) / 2} b_{i}(t)^{2} \mathrm{~d} \Omega_{2, k_{i}}^{2}\left(y_{i}^{1}, y_{i}^{2}\right) \quad \text { ( } n \text { even) }
$$

where the submanifolds $\left\{y_{i}^{1}\right.$ constant, $y_{i}^{2}$ constant $\}$ clearly have a four-dimensional FLRW line element.

- Similarly in odd dimensions, take all $p_{i}=2$, i.e., the isotropy group $S O(2)^{(n-1) / 2}$. An example is the line element

$$
\mathrm{d} s^{2}=-\mathrm{d} t^{2}+\sum_{i=1}^{(n-1) / 2} a_{i}(t)^{2} \mathrm{~d} \Omega_{2, k_{i}}^{2}\left(v_{i}^{1}, v_{i}^{2}\right) \quad(n \text { odd }) .
$$

In the case where all the $a_{i}(t)$ coincide, metric (61) is a special subcase of (48) with (a), thus comprising Einstein spacetimes (51) in particular.

One can easily construct other examples admitting different isotropies compatible with proposition 3.25. Notice that the above proposition could also be reexpressed in terms of symmetries of the Weyl tensor alone, since the proof does not rely on the presence of isometries. Other theorems regarding Weyl tensors with large symmetry groups were deduced in [16] and serve to produce further examples of PE spacetimes.

We already mentioned that only zero Weyl tensors (and thus conformally flat spacetimes) can admit $S O(n-1)$ isotropy (theorem 7.1 of [16])). Next, theorem 7.2 of [16] states that

Proposition 3.26. If the Weyl tensor of a spacetime of dimension $n>4$ admits $S O(n-2)$ isotropy, then it is of type $O$ or $D(b c d)$, and thus PE.

Remark 3.27. The statement of the proposition is no longer valid for $n=4$, the counterexamples being then precisely all non-PE Petrov type D spacetimes (any fourdimensional type D Weyl tensor has boost isotropy in the plane spanned by the PNDs, and spin isotropy in the plane orthogonal to it [9]). For instance, Petrov type D Einstein spacetimes (such as the Kerr solution), or their aligned Einstein-Maxwell 'electrovac' generalizations [9] are generically not PE (see also remarks 3.18 and 3.20). We also observe that a metric whose associated Weyl tensor is of Petrov type D (which thus admits the above mentioned isotropies) is itself, nevertheless, generically anisotropic. However, even if the spacetime itself (and not only the Weyl tensor) is $S O(2)$-isotropic (i.e., LRS [9]) then it is still not necessarily PE: 
the LRS class I and III metrics are generically not PE (nor PM; see [54] for the PE and PM conditions).

Yet, the LRS class II metrics, i.e., those admitting spherical, hyperbolical or planar symmetry (in addition to the $S O(2)$ metric isotropy), are all $P E$, just as their higher-dimensional generalizations

$$
\mathrm{d} s^{2}=F(t, x)^{2}\left(-\mathrm{d} t^{2}+\mathrm{d} x^{2}\right)+G(t, x)^{2} \mathrm{~d} \Omega_{n-2, k}^{2} \quad(n \geqslant 4) .
$$

For $n>4$ these are examples of the above proposition where (both the Weyl tensor and) the metric itself admits $S O(n-2)$ isotropy, and are special instances of proposition 3.21 (they include, in particular, the Schwarzschild(-Tangherlini) metric, and its generalizations to include a cosmological constant and/or electric charge).

For arbitrary $n$ an $S O(n-3)$ isotropic Weyl tensor does not require the spacetime to be PE (nor PM), in general: take, for instance, the five-dimensional Myers-Perry spacetime (and, more generally, see theorem 7.4 of [16]).

Finally, we note that the $2 k+1$-dimensional spacetimes with $U(k)$-symmetry $(k>1)$ given in theorem 7.5 of [16] are also PE and 'PM', in the terminology of remark 3.6.

3.5.4. Higher-dimensional 'Bianchi type I' spacetimes. We can generalize the well-known Bianchi type I spacetimes to $n$-dimensions by a spacetime allowing for $(n-1)$-dimensional spacelike hypersurfaces, $\Sigma_{t}$, possessing a transitive isometry group equal to the Abelian $\mathbb{R}^{n-1}$. Such spacetimes will also be PE:

Proposition 3.28. An n-dimensional spacetime possessing an Abelian $\mathbb{R}^{n-1}$ group of isometries acting transitively on spacelike hypersurfaces is $P E$.

Proof. Let us present two different proofs of this. First, consider the family of spatial hypersurfaces, $\Sigma_{t}$, defined as the orbits of the Abelian $\mathbb{R}^{n-1}$. We choose $\mathbf{u}$ to be the Gaussian normal to $\Sigma_{t}$. Consequently, $\mathbf{u}$ is vorticity-free, $u_{[a ; b]}=0$ and geodesic, $u^{b} u_{a ; b}=0$. Using equation (C.12) in appendix we see that the magnetic components of Weyl reduce to:

$$
C^{d g}{ }_{b c} u_{d} h_{e}^{b} h_{f}^{c}=2 h^{a g} h_{e}^{b} h_{f}^{c} \sigma_{a[b ; c]}+\frac{2}{n-2} h_{[e}^{g} h_{f]}^{b} \sigma_{b ; a}^{a} .
$$

Choosing a $\mathbf{u}$-ONF consisting of left-invariant spatial vectors, $\mathbf{m}_{\hat{i}}$ in $\Sigma_{t}$ in the standard way [62-65], the commutators satisfy $\left[\mathbf{m}_{\hat{i}}, \mathbf{m}_{\hat{j}}\right]=0$ due to the fact that $\mathbb{R}^{(n-1)}$ is Abelian. In addition, $\left[\mathbf{u}, \mathbf{m}_{i}\right]$ is tangent to the hypersurfaces due to the fact that this is an ONF. This further implies that the following connection coefficients are zero: $u_{a} \Gamma_{b c}^{a} u^{c}=\Gamma_{\hat{j} \hat{k}}^{\hat{i}}=0$. An explicit computation now gives that $h^{a g} h_{e}^{b} h_{f}^{c} \sigma_{a[b ; c]}=0$ and $\sigma_{b ; a}^{a}=0$; consequently, this spacetime is PE.

A second proof of proposition 3.28 can also be given using symmetries. The Abelian $\mathbb{R}^{n-1}$ implies also that we can, in a suitable frame, write the metric as:

$$
\mathrm{d} s^{2}=-\mathrm{d} t^{2}+\sum_{i=1}^{(n-1)} a_{i}(t)\left(\mathrm{d} x^{i}\right)^{2},
$$

where $\mathrm{d} t$ is the dual 1 -form to the Gaussian normal vector $\mathbf{u}$ above. Here, it is obvious that the discrete map $\phi:\left(t, x^{i}\right) \mapsto\left(t,-x^{i}\right)$ is an isotropy for a point with $x^{i}=0$. Since this space is spatially homogeneous, this $\phi$ extends to an isotropy at any point in space. Consider the point $p$ at the origin of $\Sigma_{t}$. Then it is straightforward to see that $\phi$ gives rise to the map $\phi^{*}=-\theta$ on $T_{p}^{*} M$, where $\theta$ is the Cartan involution. Since this is an isotropy at any point, this must extend to an isotropy of the Weyl tensor $C$ as well as all other curvature tensors. Since 
$\phi^{*}=-\theta$, on $T_{p}^{*} M$ this implies that for a curvature tensor, $T$, of rank $N$, we have the condition $(-1)^{N} \theta(T)=T$. Hence, for the Weyl tensor, which is of rank $4, \theta(C)=C$, and consequently, $C=C_{+}$and thus PE.

Examples of such spacetimes have been considered in arbitrary dimensions, for example, in [66] (here, the full group of discrete symmetries was considered).

3.5.5. Type D spacetimes with more than two multiple WANDs. Higher-dimensional type D spacetimes with more than two multiple WANDs are PE (see remark 3.15). For instance, in [33] it was proved that all type D Einstein spacetimes which admit a non-geodesic field of multiple WANDs over a region necessarily possess more than two multiple WANDs at each point of that region, and all five-dimensional such spacetimes were explicitly listed. See also $[31,34]$ for more explicit examples.

\subsection{PM spacetimes}

Contrary to PE spacetimes, properly PM spacetimes are most elusive. For instance, in four dimensions the only known Petrov type D PM spacetimes are LRS and were obtained in [54]. For $n=4$ we refer to $[67,68]$ for recent deductions of Petrov type $\mathrm{I}\left(M^{\infty}\right)$ and $\mathrm{I}\left(M^{+}\right)$PM spacetimes (cf remark 3.8), and to [17] for a complete overview of the PM literature prior to these investigations. Here we underline the elusiveness of PM spacetimes in any dimension, by proving propositions 3.29 and 3.31; this also supports the soundness of the Weyl PM definition. However, the work of [67] will enable us to construct examples of higher-dimensional (nonvacuum) PM spacetimes in section 4.3.2.

3.6.1. Restrictions on Einstein spacetimes. In a frame approach to four- or higherdimensional General Relativity, the requirement of a spacetime to obey certain geometric conditions puts constraints on the closed Einstein-Ricci-Bianchi system of equations. This may give rise to severe integrability conditions, leading to non-existence or uniqueness results. Regarding the PM condition in four dimensions it was shown, e.g., in [27] that PM, Petrov type D, aligned perfect fluids, i.e., for which the Weyl tensor is PM w.r.t. the fluid velocity, are necessarily LRS and thus also comprised in the work of [54]. As another example, aligned PM irrotational dust spacetimes have been shown not to exist, irrespective of the Petrov type [69]. In the same line severe integrability conditions arise for PM Einstein spacetimes (including the Ricci-flat case), and up to now no such solution has been found, in any dimensions. For $n=4$ it was therefore conjectured in [5] that no congruence of observers in an Einstein spacetime exist which measures the Weyl tensor to be PM. Up to present a general proof has not been found, but the validity of the conjecture was shown under a variety of additional assumptions (see again [17] for an overview), among which the Weyl type D assumption [5, 70]. This last result can be generalized to arbitrary dimension.

Proposition 3.29. In any dimension, Einstein spacetimes with a type D, PM Weyl tensor do not exist.

Proof. Assume that a PM type D Einstein spacetime exists. Take a null frame $\left\{\ell, \mathbf{n}, \mathbf{m}_{\hat{i}=3, \ldots, n}\right\}$ for which $\ell$ and $\mathbf{n}$ span the (unique) double WANDs. We work with the generalization of the Geroch-Held-Penrose formalism introduced in [71]. In the notation of [71], the PM type D Einstein space conditions translate into the vanishing of all curvature tensor components, except for $\Phi_{\hat{i} \hat{j}}^{A}=\Phi_{[\hat{i} \hat{j}]} \neq 0$ and possibly $\phi_{\hat{k} \hat{k}}=\phi=\Lambda$ (no summation over $\hat{k}, \Lambda$ being the 
cosmological constant up to normalization). We denote $\boldsymbol{\Phi}$ for the matrix $\left[\Phi_{\hat{i} \hat{j}}^{A}\right]$, and $\mathbf{S} \equiv\left[\rho_{(\hat{i} \hat{j})}\right]$ and $\mathbf{A} \equiv\left[\rho_{[\hat{i} \hat{j}]}\right]$ for the symmetric, resp. antisymmetric part of the matrix $\rho=\left[\rho_{\hat{i} \hat{j}}\right]$. Since $\Phi \neq 0$ both double WANDs are geodetic by proposition 6 of [43], such that we can take the simplified Ricci (NP) and Bianchi equations displayed in appendix A of [71]; below we shall refer to these equations by (A.x [71]) in order to avoid confusion with equations of appendix A of the present paper.

By considering the symmetric part of (A.10 [71]), and the symmetric and antisymmetric parts of the $j k$-contraction of (A.8 [71]) we immediately get

$$
\begin{aligned}
& \boldsymbol{\Phi}=\boldsymbol{S} \boldsymbol{\Phi}, \quad \boldsymbol{\Phi} \boldsymbol{A}=-\boldsymbol{A} \boldsymbol{\Phi}, \\
& \boldsymbol{\Phi}\left(\boldsymbol{S}+2 \boldsymbol{A}-\frac{1}{2} \rho \mathbf{1}_{n-2}\right)=0, \quad \rho \equiv \rho_{\hat{i}}^{\hat{i}}=S_{\hat{i}} .
\end{aligned}
$$

Let $2 p \geqslant 2$ be the rank of the antisymmetric matrix $\boldsymbol{\Phi}$. Then, by rotation of the $\mathbf{m}_{\hat{i}}$ we can put $\Phi$ in normal $2 \times 2$ block form $\left[\begin{array}{ll}x & 0 \\ 0 & 0\end{array}\right]$, where $x$ is an antisymmetric, two-block diagonal, invertible $2 p \times 2 p$ matrix. Write $\boldsymbol{S}$ and $\boldsymbol{A}$ in the same kind of block form: $\boldsymbol{S}=\left[\begin{array}{cc}s_{1} & s_{2} \\ s_{2}^{t} & s_{3}\end{array}\right], \boldsymbol{A}=\left[\begin{array}{ccc}a_{1} & a_{2} \\ -a_{2}^{t} & a_{3}\end{array}\right]$, where $y^{t}$ is the transpose of $y$, and $s_{1}$ and $s_{3}$ are symmetric whereas $a_{1}$ and $a_{3}$ are antisymmetric. Performing the matrix multiplication in (65) in $2 \times 2$ block form and using the invertibility of $x$ one gets

$$
s_{1}+2 a_{1}-\frac{\rho}{2} \mathbf{1}_{2 p}=0, \quad s_{2}+2 a_{2}=0 \quad \Rightarrow \quad s_{2}=a_{2}=0, \quad a_{1}=0, \quad s_{1}=\frac{1}{2} \rho \mathbf{1}_{2 p},
$$

by taking symmetric and antisymmetric parts. Next, taking $\hat{i}=1, \hat{j}=2$ and $\hat{k}, \hat{l}>2 p$ in (A.11 [71]) produces $a_{3}=0$, whence $\mathbf{A}=0$. Now (A.12 [71]), with $\hat{j}=1, \hat{k}=2$ gives $S_{\hat{i} \hat{l}}=0, \forall \hat{i}$ and $\forall \hat{l}>2$, implying that either $\mathbf{S}=0$, or $p=1$ and $s_{3}=0$ (notice that the latter is compatible with the last equation in (66) and $\left.\rho=S_{\hat{i}}^{\hat{i}}=\left(s_{1}\right)_{\hat{i}}^{\hat{i}}\right)$. We conclude that $\boldsymbol{\rho}=\left[\begin{array}{cc}\frac{\rho}{2} \mathbf{1}_{2} & 0 \\ 0 & 0\end{array}\right]$. Priming the above reasoning leads to $\boldsymbol{\rho}^{\prime}=\left[\begin{array}{cc}\frac{\rho^{\prime}}{2} \mathbf{1}_{2} & 0 \\ 0 & 0\end{array}\right]$, such that

$$
\rho \rho^{\prime}=\rho^{\prime} \rho \text {. }
$$

Put $T_{i} \equiv \tau_{\hat{i}}-\tau_{\hat{i}}^{\prime}$. Adding (A.13 [71]) to its prime dual gives

$$
\Phi_{\hat{i} \hat{j} \hat{j}} T_{\hat{k}]}-T_{\hat{i}} \Phi_{\hat{j} \hat{k}}=0 \text {. }
$$

Tracing over $\hat{i}$ and $\hat{k}$ leads to $T^{\hat{i}} \Phi_{\hat{i} \hat{j}}=0$; contracting now (68) with $T^{\hat{i}}$ implies $T_{\hat{i}}=0$, i.e., $\tau_{\hat{i}}=\tau_{\hat{i}}^{\prime}$. Finally, subtracting (A.6 [71]) from (A.5 [71]), using (67) and taking the antisymmetric part yields the desired contradiction $\boldsymbol{\Phi}=0$.

3.6.2. PM direct products. Similarly as in the PE case above, we now deduce necessary and sufficient conditions for a product spacetime $M^{(n)}=M^{\left(n_{1}\right)} \times M^{\left(n_{2}\right)}$ to be (properly) PM. Again, the results can be translated immediately to, e.g., warped spacetimes. We use the notation and conventions of section 3.5.2.

Firstly, recall that for $n_{1} \leqslant 2$ a direct product is PE, and thus cannot be properly PM by the first sentence of proposition 3.13. Secondly, suppose that $M^{(n)}$ is properly PM w.r.t. $\mathbf{u}=\cosh \gamma \mathbf{U}+\sinh \gamma \mathbf{Y}$, where $\mathbf{U}$ and $\mathbf{Y}$ live in the respective factor spaces. Consider the vector $\mathbf{y}=\cosh \gamma \mathbf{Y}+\sinh \gamma \mathbf{U}$ and the composite $\mathbf{u}-\mathrm{ONF}\left\{\mathbf{u}, \mathbf{m}_{A}, \mathbf{y}, \mathbf{m}_{\tilde{I}}\right\}$. By requiring $C_{y \tilde{I} A \tilde{J}}=0$ and $C_{y A B C}=0$ and using equations (8)-(10) of [43] one finds $\sinh (\gamma) R_{U A}^{\left(n_{1}\right)}=\sinh (\gamma) C_{U A B C}^{\left(n_{1}\right)}=0$. If $\sinh \gamma \neq 0$ it would follow from proposition 3.22 that $M^{(n)}$ is PE w.r.t. $\mathbf{U}$, again in contradiction with proposition 3.13. Thus $\mathbf{u}=\mathbf{U}$ (i.e., $\gamma=0$ ). Thirdly, we state the following lemma, which is proved by simple substitution in equations (9)-(11) of [43]; here and henceforth a composite $\mathbf{U}-\mathrm{ONF}\left\{\mathbf{U}, \mathbf{m}_{i=2, \ldots, n}\right\}=\left\{\mathbf{U}, \mathbf{m}_{A}, \mathbf{m}_{I}\right\}$ is used. 
Lemma 3.30. Let $M^{(n)}=M^{\left(n_{1}\right)} \times M^{\left(n_{2}\right)}$ be a direct product spacetime with $n_{1} \geqslant 3$ and $\mathbf{U} a$ unit timelike vector living in $M^{\left(n_{1}\right)}$. If

- the Ricci tensor of $M^{\left(n_{1}\right)}$ is of the form

$$
\left.R_{a b}^{\left(n_{1}\right)}=\frac{\mathcal{R}^{\left(n_{1}\right)}}{n_{1}} g_{a b}^{\left(n_{1}\right)}+U_{(a} q_{b)}, \quad U^{a} q_{a}=0 \quad \text { (i.e., } R_{A B}^{\left(n_{1}\right)}=\frac{\mathcal{R}^{\left(n_{1}\right)}}{n_{1}} \delta_{A B}, \quad R_{U U}^{\left(n_{1}\right)}=-\frac{\mathcal{R}^{\left(n_{1}\right)}}{n_{1}}\right)
$$

- $M^{\left(n_{2}\right)}$ is an Einstein space:

$$
R_{a b}^{\left(n_{2}\right)}=\frac{\mathcal{R}^{\left(n_{2}\right)}}{n_{2}} g_{a b}^{\left(n_{2}\right)} \quad\left(R_{I J}^{\left(n_{2}\right)}=\frac{\mathcal{R}^{\left(n_{2}\right)}}{n_{2}} \delta_{I J}\right) ;
$$

- the Ricci scalars of the factors are related by (53),

then the only possibly non-zero Weyl components of $M^{(n)}$ are

$$
\begin{aligned}
& C_{U I A J}=-\frac{1}{n-2} R_{U A}^{\left(n_{1}\right)} \delta_{I J}, \quad C_{U A B C}=C_{U A B C}^{\left(n_{1}\right)}-\frac{2 n_{2}}{(n-2)\left(n_{1}-2\right)} g_{A[B}^{\left(n_{1}\right)} R_{C] U}^{\left(n_{1}\right)}, \\
& C_{U A U B}=C_{U A U B}^{\left(n_{1}\right)}, \quad C_{A B C D}=C_{A B C D}^{\left(n_{1}\right)}, \quad C_{I J K L}=C_{I J K L}^{\left(n_{2}\right)} .
\end{aligned}
$$

Notice that under the conditions of the lemma $\mathcal{R}^{\left(n_{1}\right)}$ is constant, as actually follows from the decomposability of the Ricci scalar [60] and equation (53) per se. We can now prove:

Proposition 3.31. A direct product spacetime $M^{(n)}=M^{\left(n_{1}\right)} \times M^{\left(n_{2}\right)}$ is PM w.r.t. a unit timelike vector $\mathbf{U}$ that lives in $M^{\left(n_{1}\right)}$ iff the following conditions hold:

(a) $M^{\left(n_{1}\right)}$ is PM w.r.t. $\mathbf{U}$ and has Ricci tensor of the form (69);

(b) $M^{\left(n_{2}\right)}$ is of constant curvature;

(c) the Ricci scalars of the factors are related by (53), i.e., $n_{2}\left(n_{2}-1\right) \mathcal{R}^{\left(n_{1}\right)}+$ $n_{1}\left(n_{1}-1\right) \mathcal{R}^{\left(n_{2}\right)}=0$.

In this case the Weyl (magnetic) components are given by (71). Moreover, if $M^{\left(n_{1}\right)} \times M^{\left(n_{2}\right)}$ is properly $P M$ w.r.t. $\mathbf{u}$ then $n_{1} \geqslant 3$ and $\mathbf{u}$ necessarily lives in $M^{\left(n_{1}\right)}$.

Proof. Above we already proved the last sentence. Conditions (a)-(c) are precisely those of the lemma, augmented by the vanishing of the right-hand sides in (72); hence (a)-(c) is sufficient for the spacetime to be PM. Conversely, suppose that $M^{(n)}$ is PM w.r.t. U, i.e., $C_{i j k l}=0$. Expressing $C_{I J}^{I J}=0$ and using equation (11) of [43] one immediately finds (53). Next, $C_{A I B J}=0$ and equation (9) of [43] yield for $A \neq B, I \neq J$ and $A=B, I=J$ that $R_{A B}^{\left(n_{1}\right)}=0$, $R_{I J}^{\left(n_{2}\right)}=0$ and $(n-1)\left(R_{A A}^{\left(n_{1}\right)}+R_{I I}^{\left(n_{2}\right)}\right)=\mathcal{R}^{\left(n_{1}\right)}+\mathcal{R}^{\left(n_{2}\right)}$, respectively. Summing the last relation over $I$ and separately over $A$, and using (53), one arrives at (69) and (70). This proves (c) and the Ricci part of (a) and (b). Using the lemma, $M^{\left(n_{1}\right)}$ must be PM and $M^{\left(n_{2}\right)}$ conformally flat since the left-hand sides in (72) vanish, and the remaining Weyl magnetic components are (71).

Notice that the PM condition for a direct product (or conformally related) spacetime is much more stringent than the PE condition, cf propositions 3.22 and 3.24. In addition, from footnote 8 and (53) it follows that a product space $M^{(n)}=M^{\left(n_{1}\right)} \times M^{\left(n_{2}\right)}$ is a properly PM Einstein space iff it is the direct product of a properly PM Ricci-flat spacetime $M^{\left(n_{1}\right)}$ and a flat $M^{\left(n_{2}\right)}$. 


\section{The Ricci and Riemann tensors: Riemann purely electric (RPE) or magnetic (RPM) spacetimes}

Similarly as done above for the Weyl tensor, one can naturally define the electric and magnetic parts of the Ricci and Riemann tensors, and deduce properties of spacetimes which possess a PE or PM (Ricci or) Riemann tensor. This is studied in the present section. The results obtained here apply to any dimension $n \geqslant 3$ (the Ricci tensor being trivially proportional to the metric for $n=2$; also recall that the Weyl tensor vanishes for $n \leqslant 3$ ).

\subsection{Definitions and PE/PM conditions}

In accordance with definition 3.3 we define:

Definition 4.1. The electric part of the Ricci (Riemann) tensor w.r.t. $\mathbf{u}$ is the tensor $\left(R_{+}\right)_{a b}$ $\left(\left(R_{+}\right)_{a b c d}\right)$. The Ricci tensor is called PE (w.r.t. u) if $R_{a b}=\left(R_{+}\right)_{a b}$. The Riemann tensor or a spacetime is called Riemann purely electric or RPE (w.r.t. u) if $R_{a b c d}=\left(R_{+}\right)_{a b c d}$. The definitions of a PM Ricci tensor and a Riemann purely magnetic (RPM) Riemann tensor or spacetime are analogous, replacing + by - .

Based on (A.35) we have, in any $\mathbf{u}-\mathrm{ONF}$, the following component relations between the different parts:

$$
\begin{aligned}
& \left(C_{+}\right)_{i j k l}=\left(R_{+}\right)_{i j k l}-\frac{2}{n-2}\left(\delta_{i[k}\left(R_{+}\right)_{l] j}-\delta_{j[k}\left(R_{+}\right)_{l] i}\right)+\frac{2 \mathcal{R}}{(n-1)(n-2)} \delta_{i[k} \delta_{l] j}, \\
& \left(C_{+}\right)_{u i u j}=\left(R_{+}\right)_{u i u j}+\frac{1}{n-2}\left\{\left(R_{+}\right)_{i j}-\left[\left(R_{+}\right)_{u u}+\frac{\mathcal{R}}{n-1}\right] \delta_{i j}\right\} \\
& \left(C_{-}\right)_{u i j k}=\left(R_{-}\right)_{u i j k}-\frac{2}{n-2} \delta_{i[k}\left(R_{-}\right)_{j] u} .
\end{aligned}
$$

It is easy to see (cf also [11]) that the independent electric Riemann components consist of $n(n-1) / 2$ components $\left(R_{+}\right)_{u i u j}$ and $n(n-1)^{2}(n-2) / 12$ components $\left(\tilde{R}_{+}\right)_{i j k l}$, while the magnetic ones of $n(n-1)(n-2) / 3$ components $\left(R_{-}\right)_{u j i k}$ (recall the index symmetries and the cyclicity).

From (73)-(75) (or (A.19) and (A.35)) it follows that

Proposition 4.2. The Riemann tensor is RPE (RPM) w.r.t. u iff both the corresponding Weyl and Ricci tensors are PE $(P M)$ w.r.t. $\mathbf{u}$, or vanish.

In four dimensions, the RPE part of this proposition was proven in [22]. For the RPM part, the focus has usually been on the weaker condition mentioned in remark 4.7 below, for which the theorem does not hold in this form.

Just as for the Weyl tensor (proposition 3.5) one can easily derive PE/PM Bel-Debever criteria for the Ricci and Riemann tensors. Regarding the latter, one can either make a separate analysis (the only difference with the Weyl tensor being that (A.34) does not hold) or use propositions 3.5 and 4.2 .

Proposition 4.3. (Ricci and Riemann PE/PM Bel-Debever criteria) Let $\mathbf{u}$ be a unit timelike vector. Then a Ricci tensor $R_{a b} \neq 0$ is

- $P E$ w.r.t. $\mathbf{u}$ iff $R_{u i}=0$ in a $\mathbf{u}-O N F$, i.e.,

$$
u_{[a} R_{b] c} u^{c}=0
$$


- $P M$ w.r.t. $\mathbf{u}$ iff $R_{u u}=R_{i j}=0$ in a $\mathbf{u}-O N F$, i.e.,

$$
R_{a b} u^{a} u^{b}=u_{[a} R_{b][c} u_{d]}=0 .
$$

A Riemann tensor $R_{a b c d} \neq 0$ is

- RPE w.r.t. $\mathbf{u}$ iff $R_{u i j k}=0 \Leftrightarrow C_{u i j k}=R_{u i}=0$ in a $\mathbf{u}-O N F$, i.e.,

$$
u_{a} g^{a b} R_{b c[d e} u_{f]}=0 \quad \Leftrightarrow \quad u_{a} g^{a b} C_{b c[d e} u_{f]}=u_{[a} R_{b] c} u^{c}=0 ;
$$

- RPM w.r.t. $\mathbf{u}$ iff $R_{i j k l}=R_{u i u j}=0 \Leftrightarrow C_{i j k l}=R_{u u}=R_{i j}=0$ in a $\mathbf{u}-O N F$, i.e.,

$$
u_{[a} R_{b c][d e} u_{f]}=R_{a b c d} u^{b} u^{d}=0 \quad \Leftrightarrow \quad u_{[a} C_{b c][d e} u_{f]}=R_{a b} u^{a} u^{b}=u_{[a} R_{b][c} u_{d]}=0 .
$$

The PE/PM criteria for the Ricci tensor can be stated alternatively in terms of conditions on the Ricci operator on tangent space:

$$
\mathrm{R}: v^{a} \mapsto R^{a}{ }_{b} v^{b} \text {, }
$$

for which the $\mathbf{R}_{+}$and $\mathbf{R}_{-}$parts w.r.t. a unit timelike $\mathbf{u}$ have the following $1+(n-1)$ block form representations in any $\mathbf{u}-\mathrm{ONF} \mathcal{F}_{u}=\left\{\mathbf{u}, \mathbf{m}_{i=2, \ldots, n}\right\}$ :

$$
\left[\mathrm{R}_{+}\right]_{\mathcal{F}_{u}}=\left[\begin{array}{cc}
-R_{u u} & 0 \\
0 & R^{s p}
\end{array}\right], \quad\left[\mathrm{R}_{-}\right]_{\mathcal{F}_{u}}=\left[\begin{array}{cc}
0 & \alpha q^{t} \\
-\alpha q & 0
\end{array}\right] .
$$

Here $\alpha \in \mathcal{F}_{M}, R^{s p}$ is a real symmetric matrix with components $\left(R^{s p}\right)_{j}^{i}=R_{i j}$ and $q$ a unit column vector $\left(q^{t} q=1\right)$. Write $\mathbf{q} \equiv q^{i} \mathbf{m}_{i} \leftrightarrow q^{a} \equiv q^{i} m_{i}{ }^{a}$. From definition 4.1, (81) and the classification of Ricci-like tensors into types A1, A2, A3 and B (see appendix A), we readily obtain

Proposition 4.4. A Ricci tensor $R_{a b} \neq 0$ is

- $P E$ w.r.t. $\mathbf{u}$ iff $\mathrm{R}$ has $\mathbf{u}$ as an eigenvector, $\mathrm{R}(\mathbf{u})=-R_{u u} \mathbf{u}$. In this case all eigenvalues of $\mathrm{R}$ are real.

- PM w.r.t. $\mathbf{u}$ iff it has the structure

$$
R_{a b}=2 \alpha u_{(a} q_{b)}, \quad \alpha \neq 0, \quad q^{a} q_{a}=1, \quad q^{a} u_{a}=0,
$$

In this case $\mathrm{R}$ has eigenvalues 0 (multiplicity $n-2$ ) and $\pm \mathrm{i} \alpha \neq 0$, with corresponding eigenvectors $\mathbf{u} \pm i \mathbf{q}$. In particular the Ricci scalar vanishes, $\mathcal{R}=0$ (in agreement with the general result of proposition A.8.)

Remark 4.5. The resemblance with proposition 3.7 is striking. From appendix A we still have that a Ricci tensor is PE iff it has a timelike eigenvector, i.e., iff it is of type Rl. In particular, in any dimension all proper Einstein spacetimes $\left(0 \neq R_{a b} \sim g_{a b}\right)$ have a properly PE Ricci tensor. In four dimensions this is also true for perfect fluids (Segre type $\{1,(111)\}$ ) and Einstein-Maxwell fields (Segre type $\{(1,1)(11)\}$ ). However, Ricci tensors of types R3 or R4 (see appendix A) have only real eigenvalues but are not PE.

A properly PM Ricci tensor is a special instance of type R2. Referring to remark 3.8, the mentioned eigenvalue properties are equivalent with a characteristic equation for $\mathrm{R}$ of the form $\left(x^{2}+\alpha^{2}\right) x^{n-2}=0$, which is equivalent to

$\operatorname{tr}\left(\mathrm{R}^{2 k-1}\right)=0, \quad \operatorname{tr}\left(\mathrm{R}^{2 k}\right)=\frac{\left(\operatorname{tr}\left(\mathrm{R}^{2}\right)\right)^{k}}{2^{k-1}}, \quad \operatorname{tr}\left(\mathrm{R}^{2}\right) \neq 0 \quad(k=1, \ldots,\lfloor(n+1) / 2\rfloor)$.

Conversely, if a Ricci operator satisfies these conditions, then it is of type $\mathrm{R} 2$ with eigenvalues 0 (multiplicity $n-2$ ) and $\pm \mathrm{i} \alpha, \alpha=\sqrt{-\operatorname{tr}\left(\mathrm{R}^{2}\right) / 2} \neq 0$. Let $\mathbf{v}_{ \pm} \equiv \mathbf{u} \pm \mathbf{i q}$ be corresponding 
eigenvectors of $\pm \mathrm{i} \alpha$ where, by multiplication with a complex scalar, we can normalize $\mathbf{u}$ to be unit timelike. Taking the real and imaginary parts of $\mathrm{R}\left(\mathbf{v}_{ \pm}\right)= \pm \mathrm{i} \alpha \mathbf{v}_{ \pm}$we get $R_{a b} u^{b}=-\alpha q^{a}$ and $R_{a b} q^{b}=\alpha u_{a}$. By considering $q^{a} R_{a b} u^{b}$ and in view of the symmetry of $R_{a b}$ we obtain $q^{a} q_{a}=1$. However, in general $\mathbf{u}$ and $\mathbf{q}$ are not orthogonal, but if they are then (82) holds. We conclude that a Ricci tensor is properly PM iff (83) holds and the real and imaginary parts of an eigenvector with non-zero eigenvalue are orthogonal.

Remark 4.6. Replacing the Weyl by the Riemann tensor in (30) one gets the definition of the Riemann bivector operator. From (A.35) and the above results for the Ricci operator it is easy to check that proposition 3.7 still holds when replacing the Weyl by the Riemann tensor (the proof of the proposition being independent of the trace-free property (A.34)).

Remark 4.7. (a) In the four-dimensional literature, a spacetime has been called 'RPM' or 'RPE' (the quotes being part of the name) if

$$
R_{a c b d} u^{c} u^{d}=0, \quad \frac{1}{2} \varepsilon_{a c e f} R_{b d}^{e f} u^{c} u^{d}=0,
$$

respectively (see, e.g., [52, 23]). In a u-ONF these become the respective sets of conditions $R_{u i u j}=0$ and $R_{u i j k}=0$. Hence, 'RPE' coincides with our RPE notion, whereas this is not the case for 'RPM': there are no restrictions on $R_{i j k l}$ in the first of (84), i.e., it does not cover the $u_{[a} R_{b c][d e} u_{f]}=0$ part of (79), whence 'RPM' is weaker than RPM (in the terminology of $[10,11]$, the EE part of the Riemann (Weyl) tensor vanishes, but not necessarily the HH part, cf remark 3.2(b)). This is analogous to the 'PM' notion for the Weyl tensor (remark 3.6).

(b) From (74) one immediately deduces the following generalization of theorem 5 in [23] from four to arbitrary dimensions, wherein we also define a 'PM' Ricci tensor.

Proposition 4.8. Any two of the following three conditions imply the third:

(i) the Riemann tensor is 'RPM', i.e., $R_{a c b d} u^{c} u^{d}=0\left(R_{u i u j}=0\right)$;

(ii) the Weyl tensor is ' $P M$ ', i.e., $C_{a c b d} u^{c} u^{d}=0\left(C_{u i u j}=0\right)$;

(iii) the Ricci tensor is ' $P M$ ', i.e., it has the form

$$
R_{a b}=u_{(a} q_{b)}+\frac{\mathcal{R}}{n-1} h_{a b}, \quad u^{a} q_{a}=0 \quad\left(R_{u u}=0, R_{i j}=\frac{\mathcal{R}}{n-1} \delta_{i j}\right) .
$$

Hence, by comparison of (82) with (85) a Ricci tensor is PM iff it is 'PM' with vanishing Ricci scalar. In four dimensions, the spacetimes satisfying (i)-(iii), dubbed 'Haddow magnetic' [67], are Weyl PM and 'RPM', but not RPM (in general). In [67] a family of such spacetimes was deduced, the RPM members being given by the metrics (92) below and giving rise to RPM spacetimes in higher dimensions (section 4.3.2). Examples satisfying (i) but not (ii), and vice versa, were discussed in [52].

(c) Whereas the conjunction of the RPE and RPM conditions (even w.r.t. different timelike directions, see propositions 3.13 and 4.2 below) only leads to flat spacetime, the 'RPE' and 'RPM' conditions can be realized w.r.t. the same $\mathbf{u}$. This occurs iff

$$
R_{a b c d} u^{d}=0 \quad\left(\Leftrightarrow R_{u i j k}=0=R_{u i u j} \text { in a } \mathbf{u}-\mathrm{ONF}\right) .
$$

Vacuum spacetimes $R_{a b}=0$ satisfying (86) are flat in four dimensions (since $C_{u i j k}=0 \Leftrightarrow$ $H_{a b}=0$ and $C_{u i u j}=0 \Leftrightarrow E_{a b}=0$ ) but can be non-trivial in five or higher dimensions (see [15] and section 4.3.1 below). Finally notice that the trace of (86) gives $R_{u a}=0$ such that proper Einstein spacetimes $\left(0 \neq R_{a b} \sim g_{a b}\right)$ are not allowed. (See also point (ii) of section 4.3.1.) 


\subsection{Null alignment types}

For the Ricci and Riemann tensors we immediately get the following analogue of proposition 3.9.

Proposition 4.9. A Ricci tensor is $P E / P M$ w.r.t. $\mathbf{u}=(\boldsymbol{\ell}-\mathbf{n}) / \sqrt{2}$ iff in some $\mathbf{u}$-adapted null frame $\left\{\mathbf{m}_{0}=\ell, \mathbf{m}_{1}=\mathbf{n}, \mathbf{m}_{\hat{i}=3, \ldots, n}\right\}$ the following relations hold (in which case they hold in any such frame):

$$
\begin{array}{ll}
\text { PE: } & R_{00}=R_{11}, \quad R_{0 \hat{i}}=R_{1 \hat{i}} ; \\
\text { PM: } & R_{00}=-R_{11}, \quad R_{0 \hat{i}}=-R_{1 \hat{i}}, \quad R_{\hat{i} \hat{j}}=R_{01}=0 .
\end{array}
$$

A Riemann tensor is RPE (RPM) w.r.t. $\mathbf{u}=(\boldsymbol{\ell}-\mathbf{n}) / \sqrt{2}$ iff in some $\mathbf{u}$-adapted null frame the relations (38) and (87) ((39) and (88)) hold (in which case they hold in any such frame).

From the beginning of section 3.4.1 it follows that the only admitted alignment types of a PE or PM Ricci tensor, and of a RPE or RPM Riemann tensor, are G, $\mathrm{I}_{i}$, D or O (in the terminological convention at the end of section A.3 of the appendix). However, we will see that this can be further constrained in the (R)PM case. Also, if the type is $\mathrm{I}_{i}$ or $\mathrm{G}$ then the vector $\mathbf{u}$ realizing the $(\mathrm{R}) \mathrm{PE} /(\mathrm{R}) \mathrm{PM}$ property is unique, whereas it can be any vector in the Lorentzian space $\mathcal{L}_{k+2}$ if the type is D. Moreover, the properties of being properly PE and PM cannot be realized at the same time (cf proposition 3.13 and remark 3.14).

A properly PE Ricci tensor can be of any of the types G, $\mathrm{I}_{i}$ or D. For instance, it is easy to see that $R_{a b}=\alpha\left(l_{a} l_{b}+n_{a} n_{b}\right)$ (with $\alpha \neq 0$ ) is of type $\mathrm{G}$, while

$R_{a b}=\alpha_{\hat{i}}\left[\left(l_{a}+n_{a}\right) m_{b}^{\hat{i}}+m_{a}^{\hat{i}}\left(l_{b}+n_{b}\right)\right]+R_{01}\left(l_{a} n_{b}+n_{a} l_{b}\right) \quad\left(R_{01} \neq 0, \quad\right.$ at least one $\left.\alpha_{\hat{i}} \neq 0\right)$

is of type $\mathrm{I}_{i}$, i.e., the Ricci tensor has two single aligned null directions (ANDs). ${ }^{9}$ We also have

Proposition 4.10. If a Ricci-like tensor (over a vector space of dimension $n$ ) is of type I at a point, then it is of type $I_{i}$ and possesses at least a $(n-3)$-dimensional surface of single ANDs.

Proof. Recall that under a null rotation about a null vector $\mathbf{n}$ with parameter $z \equiv\left(z_{\hat{i}}\right) \equiv\left(z^{\hat{i}}\right) \in$ $\mathbb{R}^{n-2}$, a null frame $\left\{\mathbf{m}_{0}=\boldsymbol{\ell}, \mathbf{m}_{1}=\mathbf{n}, \mathbf{m}_{\hat{i}=3, \ldots, n}\right\}$ transforms to $\left\{\mathbf{m}_{0}^{\prime}=\boldsymbol{\ell}^{\prime}, \mathbf{m}_{1}^{\prime}=\mathbf{n}, \mathbf{m}_{\hat{i}=3, \ldots, n}^{\prime}\right\}$, where $\left(|z|^{2}=z^{\hat{i}} z_{\hat{i}}\right)$ :

$$
\ell^{\prime}=\boldsymbol{\ell}-z^{\hat{i}} \mathbf{m}_{\hat{i}}-\frac{|z|^{2}}{2} \mathbf{n}, \quad \mathbf{n}^{\prime}=\mathbf{n}, \quad \mathbf{m}_{\hat{i}}^{\prime}=\mathbf{m}_{\hat{i}}+z_{i} \mathbf{n} .
$$

In the new frame, the b.w. +2 component is given by

$$
R_{0^{\prime} 0^{\prime}}=R_{00}-2 z^{\hat{i}} R_{\hat{i} 0}+z^{\hat{i}} z^{\hat{j}} R_{\hat{i} \hat{j}}-|z|^{2} R_{01}+|z|^{2} z^{\hat{i}} R_{1 \hat{i}}+\frac{|z|^{4}}{4} R_{11} .
$$

If $R_{a b}$ is of type I it possesses a single AND. Let it be spanned by $\mathbf{n}$. Hence, we have $R_{11}=0$ and $\left\{R_{1 \hat{i}}\right\} \neq\{0\}$. We may rotate the spatial frame vectors such that $R_{13} \neq 0$ while all other $R_{1 \hat{i}}$ 's vanish. The null vector $\ell^{\prime}$ spans another single AND iff it satisfies the alignment equation $R_{0^{\prime} 0^{\prime}}=0$. By (91) this is a cubic equation in $z$, where the cubic term is $|z|^{2} z_{3} R_{13}$. For any fixed value $\left(z_{4}^{0}, z_{5}^{0}, \ldots, z_{n}^{0}\right) \in \mathbb{R}^{n-3}$ we get a cubic equation in $z_{3}$, which has thus at least one real solution $z_{3}^{0}$ depending continuously on $\left(z_{4}^{0}, z_{5}^{0}, \ldots, z_{n}^{0}\right)$.

9 Checking this is trivial for the type $\mathrm{G}$ example. For the type $\mathrm{I}_{i}$ example, consider a generic null rotation (90). In the new frame one finds $R_{0^{\prime} 0^{\prime}}=-z_{\hat{i}} R_{0^{\prime} \hat{i}^{\prime}}-\left(|z|^{2} / 2+1\right) z_{\hat{i}} \alpha_{\hat{i}}$ and $R_{0^{\prime} \hat{i}^{\prime}}=\alpha_{\hat{i}}\left(-z^{2} / 2+1\right)+z_{\hat{i}}\left(R_{01}-z_{\hat{j}} \alpha_{\hat{j}}\right)$. The existence of a double AND $\ell^{\prime}$ requires $R_{0^{\prime} 0^{\prime}}=0=R_{0^{\prime} \hat{i}^{\prime}}$, which leads to $\alpha_{\hat{i}} z_{\hat{i}}=0=2 z_{\hat{i}} R_{01}+\alpha_{\hat{i}}\left(2-|z|^{2}\right)$. Contracting the latter equation with $z_{\hat{i}}$ gives $|z|^{2}=0$, i.e., all $z_{\hat{i}}=0$. This then implies $\alpha_{\hat{i}}=0$, leading to a contradiction. Therefore in this case there do not exist any doubly ANDs, so that the type is indeed $\mathrm{I}_{i}$, as claimed. 
Proposition 4.11. Any type D Ricci-like tensor $R_{a b}$ is PE. For a type D PM Weyl tensor $C_{a b c d}$, any symmetrized rank 2 contraction of an odd power vanishes: $\operatorname{Tr}_{4 m+1}\left(C^{2 m+1}\right)_{(a b)}=0$.

Proof. The first statement is trivially seen by taking a null frame $\left\{\boldsymbol{\ell}, \mathbf{n}, \mathbf{m}_{\hat{i}=3, \ldots, n}\right\}$ where $\ell$ and $\mathbf{n}$ are double aligned null vectors, such that $R_{a b}=R_{01}\left(l_{a} n_{b}+n_{a} l_{b}\right)+R_{\hat{i} j} m_{a}^{\hat{i}} m_{b}^{\hat{i}}$, with $\left(R_{01},\left\{R_{\hat{i} \hat{j}}\right\}\right) \neq(0,\{0\})$. The second statement is a consequence of proposition A.8, corollary A.12 and the first statement.

From remark 3.14 it thus follows that a properly PM Ricci tensor cannot be of type D. More specifically we have

Proposition 4.12. A PM Ricci tensor is of alignment type $I_{i}$ (i.e., types $G$ and $D$ are forbidden) and has a $(n-3)$-dimensional sphere of single ANDs paired up by the unique unit timelike $\mathbf{u}$ realizing the PM condition.

Proof. Putting $q_{a}=\mathbf{m}_{a}^{2}$ in (82) and defining $\boldsymbol{\ell}$ and $\mathbf{n}$ by (1) we have

$$
R_{a b}=\alpha\left(l_{a} l_{b}-n_{a} n_{b}\right), \quad \alpha \neq 0,
$$

cf (B.1). In a null frame $\left\{\mathbf{m}_{0}=\boldsymbol{\ell}, \mathbf{m}_{1}=\mathbf{n}, \mathbf{m}_{\hat{i}=3, \ldots, n}\right\}$ the only surviving components are $R_{11}=-R_{00}=\alpha \neq 0$. Under a null rotation (90) the components of positive b.w. in the new frame are given by

$$
R_{0^{\prime} 0^{\prime}}=R_{00}+\frac{|z|^{4}}{4} R_{11}=\frac{\alpha}{4}\left(|z|^{4}-4\right), \quad R_{0^{\prime} \hat{i}^{\prime}}=-\alpha \frac{|z|^{2}}{2} z_{\hat{i}} \neq 0
$$

such that $R_{a b}$ has a continuous infinity of single ANDs given by all $\ell^{\prime}=\ell^{\prime}(z)$ satisfying the alignment equation $R_{0^{\prime} 0^{\prime}}=0$, i.e., $|z|=\sqrt{2}$. Thus the set of single ANDs is an $(n-3)$ dimensional sphere. The vector $\mathbf{u}=(\ell-\mathbf{n}) / \sqrt{2}$ appearing in (82) is the unique vector realizing the PM condition and pairs up this sphere of single ANDs.

In general, the alignment types of a RPE/RPM Riemann tensor are subject to corollary A.14. In the RPM case, combination hereof with proposition 4.12 immediately implies:

Corollary 4.13. If, at a point, a spacetime is RPM w.r.t. $\mathbf{u}$ and the Ricci tensor is non-zero, then the RPM Riemann tensor is of alignment type $I_{i}$ or $G$ (i.e., type $D$ is forbidden). In particular, $\mathbf{u}$ realizing the RPM property is always unique.

More specifically, proposition A.13 holds. For instance, if a Riemann tensor is RPM w.r.t. $\mathbf{u}$ and of type $\mathrm{I}_{i}$, then $\mathbf{u}$ can be written as $(36)$, where $b_{\text {Rie }}(\boldsymbol{\ell})=b_{\text {Rie }}(\mathbf{n})=1$, and both the corresponding Ricci and Weyl tensors are PM w.r.t. $\mathbf{u}$, where $\max \left(b_{\mathrm{Ric}}(\boldsymbol{\ell}), b_{C}(\boldsymbol{\ell})\right)=$ $\max \left(b_{\text {Ric }}(\mathbf{n}), b_{C}(\mathbf{n})\right)=1$. If a Riemann tensor is RPE w.r.t. $\mathbf{u}$ and of type $\mathrm{D}$, and the Ricci and Weyl tensors are non-zero, then vectors $\boldsymbol{\ell}$ and $\mathbf{n}$ exist for which (36) holds and along which the boost orders of the Riemann, Ricci and Weyl tensors are all zero. Finally, from proposition A.13, remark (3.15) and the fact that a type D Ricci tensor is automatically PE, it follows that if a type D Riemann tensor has more than two double ANDs then it is RPE.

\subsection{Direct products and explicit examples}

The first part of the following proposition is a restatement of proposition 3.22, while the second part is an immediate consequence of proposition 3.31.

Proposition 4.14. Let $M^{(n)}=M^{\left(n_{1}\right)} \times M^{\left(n_{2}\right)}$ be a direct product spacetime and $\mathbf{U}$ a timelike vector that lives in $M^{\left(n_{1}\right)}$. Then 
- $M^{(n)}$ is RPE w.r.t. $\mathbf{U}$ iff $M^{\left(n_{1}\right)}$ is RPE w.r.t. $\mathbf{U}$ (which is the case iff $M^{(n)}$ is PE w.r.t. $\mathbf{U}$ );

- $M^{(n)}$ is RPM w.r.t. $\mathbf{U}$ iff $M^{\left(n_{1}\right)}$ is RPM w.r.t. $\mathbf{U}$ and $M^{\left(n_{2}\right)}$ is flat.

Recall that $\mathbf{U} \in M^{n_{1}}$ is not a restriction in the (R)PE case, and is the only possibility in the $\mathrm{PM}$ or RPM cases. It is thus evident that RPE/RPM spacetimes have a special significance in the construction of higher-dimensional (R)PE or (R)PM spacetimes, e.g., from those already known in four dimensions.

Remark 4.15. Regarding proposition 3.24, one may define the electric and magnetic parts of the Weyl, Ricci and Riemann tensors of the Riemannian space $M^{\left(n_{2}\right)}$ relative to a spacelike vector $\mathbf{Y}$, analogously as for timelike vectors. Doing so, the duality in equations (55)-(56) is manifest. The first two equations in (55) and (56) tell that a direct product $M^{(n)}=M^{\left(n_{1}\right)} \times M^{\left(n_{2}\right)}$ which is (R)PE w.r.t. a unit timelike vector $\mathbf{u}$ not living in $M^{\left(n_{1}\right)}$ must have factors which are RPE w.r.t. the respective normalized projections of $\mathbf{u}$; the last two equations are relations between electric tensors and can be covariantly rewritten as

$U^{c} U^{d}\left(R_{a c b d}^{\left(n_{1}\right)}-\frac{1}{n_{1}-1} h_{a b}^{\left(n_{1}\right)} R_{c d}^{\left(n_{1}\right)}\right)=0, \quad Y^{c} Y^{d}\left(R_{a c b d}^{\left(n_{2}\right)}-\frac{1}{n_{2}-1} h_{a b}^{\left(n_{2}\right)} R_{c d}^{\left(n_{2}\right)}\right)=0$,

where $\left(h^{\left(n_{i}\right)}\right)^{a}{ }_{b}$ are the projectors in $M^{\left(n_{i}\right)}$ orthogonal to $\mathbf{U}(i=1)$ or $\mathbf{Y}(i=2)$.

4.3.1. RPE spacetimes. We mention generic conditions under which spacetimes are RPE, thereby taking section 3.5 as a thread.

(1) Spacetimes admitting a shear-free normal unit timelike vector field $\mathbf{u}$ are RPE w.r.t. $\mathbf{u}$ iff moreover the expansion scalar of $\mathbf{u}$ is spatially homogeneous, i.e., $h_{a}{ }^{b} \tilde{\Theta}_{, b}=0$. This follows from (C.6) and (C.7). Referring to (48) this is the additional condition $\tilde{\Theta}=\tilde{\Theta}(t)$; integrating the first equation in (49) this is precisely the case if $P=\mathrm{e}^{\int V\left(t, x^{\gamma}\right) \tilde{\Theta}(t) \mathrm{d} t}$ (after absorbing the function of integration into $\left.\xi\left(x^{\gamma}\right)\right)$. Then $\mathbf{u}=\partial_{t} / V$ is an eigenvector of the Ricci tensor with eigenvalue $-\dot{u}^{a} ; a+(n-1)\left(\dot{\tilde{\Theta}}+\tilde{\Theta}^{2}\right)$, see (C.10). Special instances are spacetimes admitting a non-rotating rigid $\mathbf{u}\left(\sigma_{a b}=\tilde{\Theta}=\omega_{a b}=0\right)$ and the warped spacetimes with a one-dimensional timelike factor, i.e., cases (a)-(c) in section 3.5.1. In particular, all static spacetimes are RPE. In contrast, doubly-warped spacetimes with a one-dimensional timelike factor (case (d)) are PE but never RPE w.r.t. u.

(2) Spacetimes which satisfy (86) are RPE and 'RPM', cf remark 4.7(c). Within the warped class (a) of 3.5.1, where $\dot{u}^{a}=0$ additionally, this is realized iff $\tilde{\Theta}=-\tilde{\Theta}^{2}$, see (C.6). Examples hereof are the direct product spacetimes of the subclass (c), i.e., those spacetimes admitting a covariantly constant unit timelike vector field $\mathbf{u}\left(\sigma_{a b}=\tilde{\Theta}=\omega_{a b}=\dot{u}^{a}=0\right)$, and the $n \geqslant 5$ warped spacetimes (51) with $\lambda=0$ (vacuum case).

(3) All direct or warped products (52), with a RPE timelike factor and with $\theta: M^{\left(n_{2}\right)} \rightarrow \mathbb{R}$, are RPE. This follows from proposition 4.14 and, e.g., equations (25) in [72] or (D.8) in [73]. For $n_{1}=1$ (giving case (a) of section 3.5.1) and $n_{1}=2$, the RPE condition on the timelike factor is automatically satisfied since then $R^{\left(n_{1}\right)} \sim \boldsymbol{g}^{\left(n_{1}\right)}$ (see also the top of page 4415 of [43], and cf remark 3.23). As an instance of $n_{1}=4$ RPE spacetimes we may mention aligned perfect fluids (for which the Weyl tensor is PE w.r.t. the fluid velocity u) and their Einstein space limits; for instance, all examples mentioned at the end of remark 3.18 can be lifted by the above direct or warped product construction.

(4) All spacetimes with an isotropy group mentioned in proposition 3.25 are in fact RPE (in the proof no use was made of the trace-free property (A.34) of the Weyl tensor, just as the $2 k+1$-dimensional spacetimes with $U(k)$ isotropy $(k>1))$. The spacetimes (62) with spherical, hyperbolical or planar symmetry are RPE iff the matrix $\left[\begin{array}{cc}R_{01} & R_{11} \\ R_{00} & R_{01}\end{array}\right]$ is of type R1 
in section B.1 in the appendix, relative to a null frame $\left\{\mathbf{m}_{0}=\boldsymbol{\ell}, \mathbf{m}_{1}=\mathbf{n}, \mathbf{m}_{\hat{i}=3, \ldots, n}\right\}$ where $\ell$ and $\mathbf{n}$ live in the timelike factor $M^{\left(n_{1}\right)}, n_{1}=2$ (see, e.g., equations (25) in [72]). This is precisely the case when $R_{00} R_{11}>0$ or $R_{00}=R_{11}=0$, the latter case implying Ricci type D.

(5) Higher-dimensional 'Bianchi type I' spacetimes, studied in section 3.5.4, are also RPE spacetimes. Again, this can be shown in two different ways; however, restricting to the second proof in section 3.5.4 one sees that the discrete symmetry implies for the Ricci tensor, $\theta(R)=R=R_{+}$; consequently, the spacetime is RPE.

(6) All PE Einstein spacetimes are obviously also RPE (cf remark 4.5).

4.3.2. RPM spacetimes. Evidently, RPM spacetimes are even more elusive than Weyl PM spacetimes. The only (two-parameter) class of RPM spacetimes known so far was derived in four dimensions by Lozanovski [67] (cf also remark 4.7(b)), the line element being (up to a constant rescaling).

$\mathrm{d} s_{L}^{2}=\exp (-2 b z)\left[-\mathrm{d} t^{2}+\mathrm{d} z^{2}\right]+\exp (2 a y)\left[\mathrm{d} x^{2}+t^{2} x^{2} \mathrm{~d} y^{2}\right] \quad(a, b \in \mathbb{R})$.

This spacetime, which contains an 'imperfect fluid' [67], is RPM w.r.t. u $=\exp (b z) \partial_{t}$, and of Petrov type $\mathrm{I}\left(M^{+}\right)$for all values of $a$ and $b$, except when $a b=0$, in which case the type is $\mathrm{I}\left(M^{\infty}\right)$, cf remark 3.8. According to proposition 4.14, explicit examples of higherdimensional RPM spacetimes can be produced by taking direct products with flat Euclidean spaces. Additionally, Weyl PM (but not RPM) spacetimes can be generated from such direct products by simply performing a (non-trivial) conformal transformation (under which the Weyl tensor is invariant while the Ricci tensor will lose its PM character, in general). For the sake of definiteness, consider the five-dimensional line element

$$
\mathrm{d} s^{2}=\mathrm{e}^{k z}\left(\mathrm{~d} s_{L}^{2}+\mathrm{d} w^{2}\right),
$$

with $\mathrm{d} s_{L}^{2}$ given by (92). This is a spacetime PM w.r.t. $\mathbf{u}=\mathrm{e}^{(2 b-k) z / 2} \partial_{t}$. It is, additionally, RPM (w.r.t. the same $\mathbf{u}$ ) iff it is a direct product, i.e., $k=0$ (the necessity of this follows from the last statement of proposition 4.4 and computation of the Ricci scalar $\mathcal{R}=-3 k^{2} \mathrm{e}^{z(2 b-k)}$, while the sufficiency follows from the second part of proposition 4.14). In the latter case one has $R_{\alpha \beta \gamma w}=0$, so that $\exp (b z) \partial_{t} \pm \partial_{w}$ are null directions aligned with the Riemann tensor when $k=0$ (thus, the Riemann tensor is of type $\mathrm{I}_{i}$ in this case). A fortiori, these are also WANDs (cf proposition A.13), so that the Weyl tensor cannot be of type G. Moreover, a direct computation shows that for this metric, the symmetric rank 2 tensor

$$
T_{a g}=T_{(a g)} \equiv C_{a b c d} C^{c d}{ }_{e f} C^{e f b}
$$

does not vanish. Hence, by proposition 4.11 the Weyl tensor cannot be type D. Since the case $k \neq 0$ is just obtained by a conformal transformation, it follows that all metrics (93) are of Weyl type $I_{i}$, and thus PM uniquely w.r.t. $\mathbf{u}=\mathrm{e}^{(2 b-k) z / 2} \partial_{t}$.

To our knowledge, such products are the only examples of higher-dimensional (R)PM spacetimes found so far.

\section{Conclusion and discussion}

We introduced and elaborated a twofold decomposition of any tensor at a point of a spacetime of arbitrary dimension, relative to a unit timelike vector $\mathbf{u}$. The splitting is based on considering a (time) reflection of $\mathbf{u}$, which itself is a special instance of a Cartan involution (when applied to the Lorentz group). We saw that this leads to a generalization, from four to arbitrary dimensions, of the electric/magnetic decomposition of the Maxwell and Weyl tensors. That 
this generalization is natural has been confirmed by the extension of many four-dimensional results regarding $\mathrm{PE}$ and $\mathrm{PM}$ curvature tensors to higher dimensions.

In particular, we derived a close connection between PE/PM properties and the existence of preferred null directions. Hereby we focused on the curvature tensors, so crucial in (fouror higher-dimensional) General Relativity as well as in other gravity theories. However, many of these properties generalize to arbitrary tensors and operators; as such they are applicable to any physical theory governed by tensor objects defined over a spacetime (manifold with Lorentzian metric), with the potential of leading to novel interesting viewpoints and results in such contexts.

Tensors for which one of the two parts in the splitting w.r.t. $\mathbf{u}$ vanishes are examples of tensors which are minimal w.r.t. $\mathbf{u}$, in the sense that the sum of squares of the tensor components in any $\mathbf{u}$-adapted ONF is not larger than for any other $\mathbf{u}^{\prime}$. Via a new proof of the alignment theorem we made an intriguing connection with both the null alignment and polynomial invariants properties of such tensors: these are precisely the tensors characterized by their invariants or, still, the tensors which do not possess a unique AND of boost order $\leqslant 0$. Future inquiries on these facts may be important for shedding new light on the invariant content of many modern theories (string theory, brane world models, quantum cosmology, etc). In particular, the classification of spacetimes themselves makes use of the Riemann tensor and its covariant derivatives via the Cartan-Karlhede algorithm, and thus may highly benefit from such investigations.

This paper also demonstrates the interesting link between special classes of spacetimes and invariant theory. This link is explicitly demonstrated by the connection between the Cartan involution, which is important in the classification of Lie algebras, and a simple time-reflection. This enabled us to connect these seemingly distinct areas and use the best from both worlds to prove deep results about the existence/non-existence of certain solutions. It is believed that this bond will continue to bear fruits in investigations to come.

\section{Acknowledgments}

We thank Alan Barnes for reading the manuscript and José M M Senovilla for useful comments and references. MO has been supported by research plan RVO: 67985840 and research grants GAČR P203/10/0749 and GAČR 13-10042S. LW has been supported by an Yggdrasil mobility grant no 211109 to Stavanger University, a BOF research grant of Ghent University, and a FWO mobility grant no V4.356.10N to Utrecht University, where parts of this work were performed. MO and LW thank the Faculty of Science and Technology of the University of Stavanger for its hospitality during a research stay.

\section{Appendix A. Orbits of tensors, Cartan involutions, and null alignment theory}

\section{A.1. Orbits of tensors; tensors characterized by their invariants}

Let us review some results from invariant theory and define the appropriate concepts which we need. Furthermore, we will consider polynomial invariants of tensors, and so in what follows 'invariants' is to be understood as 'polynomial invariants'.

The idea is to consider a group $G$ acting on a vector space $V$. In our case we will consider a real $G$ and a real vector space $V$. However, it is advantageous to review the complex case with a complex group $G^{\mathbb{C}}$ acting on a complex vector space $V^{\mathbb{C}}$. For a vector $X \in V^{\mathbb{C}}$, the orbit of $X$ under the action of $G^{\mathbb{C}}$ is defined as

$$
\mathcal{O}_{\mathbb{C}}(X) \equiv\left\{g(X) \in V^{\mathbb{C}} \mid g \in G^{\mathbb{C}}\right\} \subset V^{\mathbb{C}} .
$$

Then ([74, p 555-6]): 
Proposition A.1. If $G^{\mathbb{C}}$ is a linearly reductive group acting on an affine variety $V^{\mathbb{C}}$, then the ring of invariants is finitely generated. Moreover, the quotient $V^{\mathbb{C}} / G^{\mathbb{C}}$ parametrizes the closed orbits of the $G^{\mathbb{C}}$-action on $V^{\mathbb{C}}$ and the invariants separate closed orbits.

Here the term closed refers to topologically closed with respect to the standard vector space topology and henceforth, closed will mean topologically closed. This implies that given two distinct closed orbits $A_{1}$ and $A_{2}$, then there is an invariant with value 1 on $A_{1}$ and 0 on $A_{2}$. This enables us to define the set of closed orbits:

$$
\mathfrak{C}_{\mathbb{C}} \equiv\left\{\mathcal{O}_{\mathbb{C}}(X) \subset V^{\mathbb{C}} \mid \mathcal{O}_{\mathbb{C}}(X) \text { closed. }\right\}
$$

Based on the above proposition we can thus say that the invariants separate elements of $\mathfrak{C}_{\mathbb{C}}$ and hence we will say that an element of $\mathfrak{C}_{\mathbb{C}}$ is characterized by its invariants.

In our case we consider the real case where we have the Lorentz group $G=O(1, n-1)$ which is a real semi-simple group. For real semi-simple groups acting on a real vector space $V$ we do not have the same uniqueness result as for the complex case [75], see also remark A.6. However, by complexification, $[G]^{\mathbb{C}}=G^{\mathbb{C}}$ we have $[O(1, n-1)]^{\mathbb{C}}=O(n, \mathbb{C})$, and by complexification of the real vector space $V$ we get $V^{\mathbb{C}} \cong V+\mathrm{i} V$. The complexification thus lends itself to the above theorem.

Concretely, we study tensors, $T$, belonging to some tensor space $\mathcal{T}_{s}^{r} \equiv\left(T_{p} M\right)^{\otimes r} \otimes$ $\left(T_{p}^{*} M\right)^{\otimes s}$, where $p$ is a point of an $n$-dimensional manifold $M$ with Lorentzian metric $g$. Let $\boldsymbol{\omega} \equiv\left\{\mathbf{m}_{\alpha=1, \ldots, n}\right\}$ be a basis of vectors of $T_{p} M$. Let $g \in G$ be a Lorentz transformation, with representation matrix $\left(M^{\alpha}{ }_{\beta}\right)$ w.r.t. $\omega$, i.e., in the natural action of $g$ on $T_{p} M$ we have $g\left(\mathbf{m}_{\alpha}\right)=M_{\alpha}^{\beta} \mathbf{m}_{\beta}$. Consider now the following action on the components of $T$ w.r.t. $\boldsymbol{\omega}$ :

$$
T_{\beta_{1} \cdots \beta_{s}}^{\alpha_{1} \cdots \alpha_{r}} \mapsto\left(M^{-1}\right)^{\alpha_{1}}{ }_{\gamma_{1}} \cdots\left(M^{-1}\right)^{\alpha_{r}}{ }_{\gamma_{r}} T^{\gamma_{1} \cdots \gamma_{r}}{ }_{\delta_{1} \cdots \delta_{s}} M_{\beta_{1}}^{\delta_{1}} \cdots M_{\beta_{s}}^{\delta_{s}} .
$$

As is well known, the real numbers on the right-hand side may be interpreted as either

(1) the components of the original tensor $T$ w.r.t. a new basis $\left\{g\left(\mathbf{m}_{\alpha=1, \ldots, n}\right)\right\}$ of $T_{p} M$ (and the dual basis of $\left.T_{p}^{*} M\right)$, or

(2) the components w.r.t. the original basis $\left\{\mathbf{m}_{\alpha=1, \ldots, n}\right\}$ of a new tensor $T^{\prime}$, which is the result of $g^{-1}$ acting as a tensor map on $T \in \mathcal{T}_{s}^{r}$.

In the former case one puts the components of $T$ in a vector $v \in V=\mathbb{R}^{m}, m=n^{r+s}$, and one speaks about the passive action of $O(1, n-1)$ on $V$; notice that $V$ has an $(r, s)$-tensor structure as well here (over $\mathbb{R}^{n}$ instead of $T_{p} M$ ). In the latter case one considers $T \mapsto T^{\prime}$, referred to as the active action of $g^{-1} \in O(1, n-1)$ on $V=\mathcal{T}_{s}^{r}$. It is clear that both viewpoints are essentially equivalent, although one of them may be more natural in a specific context. In either picture we may consider a collection (or direct sum) of tensors instead of a single one (which just changes $V$ accordingly).

Based on the above, tensors 'characterized by invariants' are defined as follows, in the passive viewpoint.

Definition A.2. Consider a (real) tensor, $T$, or a direct sum of tensors, and let $\tilde{T} \in V$ be the corresponding vector of components w.r.t. a certain basis. If the orbit of $\tilde{T}$ under the complexified Lorentz group $G^{\mathbb{C}}$ is an element of $\mathfrak{C}_{\mathbb{C}}$, i.e., $\mathcal{O}_{\mathbb{C}}(\tilde{T}) \in \mathfrak{C}_{\mathbb{C}}$, then we say that $T$ is characterized by its invariants.

As the invariants parametrize the set $\mathfrak{C}_{\mathbb{C}}$ and since the group action defines an equivalence relation between elements in the same orbit this definition makes sense.

In analogy with (A.1) and (A.2) let us define the real orbit through $X$ and the set of real closed orbits:

$$
\mathcal{O}(X) \equiv\{g(X) \in V \mid g \in G\} \subset V,
$$




$$
\mathfrak{C} \equiv\{\mathcal{O}(X) \subset V \mid \mathcal{O}(X) \text { closed }\}
$$

How do the results of proposition A.1 translate to the real case? A real orbit $\mathcal{O}(X)$ is a real section of the complex orbit $\mathcal{O}_{\mathbb{C}}(X)$. However, there might be more than one such real section having the same complex orbit. Using the results of [75], these real closed orbits are disjoint, moreover:

Proposition A.3. $\mathcal{O}(X)$ is closed in $V \Leftrightarrow \mathcal{O}_{\mathbb{C}}(X)$ is closed in $V^{\mathbb{C}}$.

Combining this with proposition A.1 and definition A. 2 we thus have

Corollary A.4. A tensor $T$ is characterized by its invariants iff its orbit is closed in $V$, $\mathcal{O}(\tilde{T}) \in \mathfrak{C}$.

Remark A.5. The case of a direct sum of curvature tensors, i.e., the Riemann tensor and its covariant derivatives, is of particular importance for the equivalence problem of metrics (of arbitrary signature). Let $\tilde{X}=\tilde{R}_{\boldsymbol{\omega}} \equiv\left[R_{\alpha \beta \gamma \delta}, R_{\alpha \beta \gamma \delta ; \epsilon}, \ldots, R_{\alpha \beta \gamma \delta ; \epsilon_{1} \cdots \epsilon_{k}}\right] \in \mathbb{R}^{m(k)}$ be the vector of components w.r.t. a (for instance orthonormal) frame $\boldsymbol{\omega}=\left\{\mathbf{m}_{\alpha=1, \ldots, n}\right\}$, at a point $p$ of a manifold $M$ with metric $g$, of the curvature tensors up to the $k$ th derivative, where $m(k)=n^{4}\left(n^{k+1}-1\right) /(n-1)$. Then the action of $g \in O(1, n-1)$ on $\tilde{X}$ is $g(\tilde{X})=\left[M_{\alpha}^{\kappa} \cdots M_{\delta}^{v} R_{\kappa \cdots \nu}, M_{\alpha}^{\kappa} \cdots M_{\delta}^{v} M^{\xi}{ }_{\epsilon} R_{\kappa \cdots \nu ; \xi}, \ldots, M_{\alpha}^{\kappa} \cdots M_{\delta}^{v} M^{\xi_{1}} \epsilon_{1} \cdots M^{\xi_{k}} \epsilon_{k} R_{\kappa \cdots \nu ; \xi_{1} \cdots \xi_{k}}\right]$.

Let $\tilde{Y}=\tilde{R}_{\boldsymbol{\omega}^{\prime}}^{\prime} \in \mathbb{R}^{m(k)}$ be the analogous curvature vector for a metric $\boldsymbol{g}^{\prime}$ on $M$, w.r.t. a frame $\boldsymbol{\omega}^{\prime}$ at $p$. Then, if $\tilde{X}$ and $\tilde{Y}$ are in the same real orbit, we have $\tilde{Y}=g(\tilde{X})$ for certain $g \in O(1, n-1)$, i.e., the respective representation vectors $\tilde{X}$ and $\tilde{Y}$ are separated by a mere rotation of frame, $\boldsymbol{\omega}^{\prime}=g(\boldsymbol{\omega})$. If this holds for $k=n(n+1) / 2$ at every point $p$ of a local neighborhood $U$ of $M$, then a result of Cartan (see e.g. [9]) tells that $\boldsymbol{g}$ and $\boldsymbol{g}^{\prime}$ are equivalent on $U$. In this way the equivalence problem is reduced to a question of classifying the various orbits.

Remark A.6. As pointed out, different closed real orbits $\mathcal{O}(T)$ may have the same invariants (in line with the comments in [76, 77]). An example of this is given by the pair of metrics, clearly related by a double Wick rotation [77]:

$$
\begin{aligned}
& \mathrm{d} s_{1}^{2}=-\mathrm{d} t^{2}+\frac{1}{x^{2}}\left(\mathrm{~d} x^{2}+\mathrm{d} y^{2}+\mathrm{d} z^{2}\right), \\
& \mathrm{d} s_{2}^{2}=\mathrm{d} \tau^{2}+\frac{1}{x^{2}}\left(\mathrm{~d} x^{2}+\mathrm{d} y^{2}-\mathrm{d} \zeta^{2}\right) .
\end{aligned}
$$

These metrics are symmetric $\left(R_{a b c d ; e}=0\right)$ and conformally flat; hence, the Riemann tensor is the only non-zero curvature tensor and is equivalent to the Ricci tensor. In both cases, at any spacetime point, the Ricci operator $R_{b}^{a}$ acting on tangent space has a single eigenvalue 0 and a triple eigenvalue -2 (the spacetimes being homogeneous), such that the respective Ricci tensors have the same polynomial invariants and belong to the same complex orbit $\mathcal{O}_{\mathbb{C}}(T)$. However, the Segre type of $R^{a}{ }_{b}$ is $\{1,(111)\}$ for the former and $\{(1,11) 1\}$ for the latter metric; thus the respective Ricci tensors lie in separate real orbits $\mathcal{O}(T)$.

\section{A.2 Cartan involutions of the Lorentz group}

A.2.1 Representation on tensor spaces. Consider the full Lorentz group $G=O(1, n-1)$. Let $K \cong O(n-1)$ be a maximal compact 'spin' subgroup of $O(1, n-1)$. Then there exists a unique Cartan involution $\theta$ of $O(1, n-1)$ with the following properties [14]:

(i) $\theta$ is invariant under the adjoint action of $K$ :

$$
A d_{K}(\theta)=\theta, \quad \text { i.e., } \quad k \theta=\theta k, \quad \forall k \in K \text {; }
$$


(ii) $O(1, n-1)$ is $\theta$-stable, $\theta(O(1, n-1))=O(1, n-1)$;

(iii) $\theta$ is the automorphism $X \mapsto-X^{*}$ of the Lie algebra $\mathfrak{g l}(n, \mathbb{R})$, where * denotes the adjoint (or transpose, since the coefficients are real).

In general, the maximal subgroups of a semi-simple Lie group $G$ are all conjugate, such that two Cartan involutions are related by $\theta_{2}=\operatorname{Int}(g) \theta_{1} \operatorname{Int}\left(g^{-1}\right)$, where $\operatorname{Int}(g)$ is the inner automorphism by a certain $g \in G$.

In our case, consider the natural representation of $G=O(1, n-1)$ on the tangent space $T_{p} M$ at a point $p$ of a Lorentzian manifold $(M, g)$. Then, any maximal compact subgroup $K$ is in biunivocal relation with the timelike direction which is invariant under the action of $K$. If this direction is spanned by the unit timelike vector $\mathbf{u}$, then it is easy to see that the unique Cartan involution corresponding to $K$ is simply the reflection

$$
\theta: \quad \mathbf{u} \mapsto-\mathbf{u}, \quad \mathbf{x} \mapsto \mathbf{x}, \quad \forall \mathbf{x} \perp \mathbf{u},
$$

acting as an inner automorphism on $G .{ }^{10}$ Thus $\theta$ can be seen as a Lorentz transformation itself, with action (A.8) on $T_{p} M$. In any $\mathbf{u}$-ONF $\mathcal{F}_{u}=\left\{\mathbf{m}_{1}=\mathbf{u}, \mathbf{m}_{i=2, \ldots, n}\right\}$ we have the matrix representation:

$$
[\theta]_{\mathcal{F}_{u}}=\left(\theta^{\alpha}{ }_{\beta}\right)=\operatorname{diag}(-1,1, \ldots, 1) .
$$

Obviously in such a frame $\theta$ is simply a time reversal transformation. In abstract index notation we have

$$
\delta_{b}^{a}=g^{a}{ }_{b} \equiv h^{a}{ }_{b}-u^{a} u_{b}, \quad \theta^{a}{ }_{b}=h^{a}{ }_{b}+u^{a} u_{b},
$$

where the first part defines the projector $h^{a}{ }_{b}$ of $T_{p} M$ orthogonal to $\mathbf{u}, \delta_{b}^{a}$ being the identity transformation.

Through the tensor map construction $\theta$ acts as a reflection $\left(\theta^{2}=1\right)$ on any tensor space $V=\mathcal{T}_{s}^{r}$ by (A.3), with $M^{\alpha}{ }_{\beta}=\left(M^{-1}\right)^{\alpha}{ }_{\beta}=\theta^{\alpha}{ }_{\beta}$ (we adopt the active viewpoint here and, with a slight abuse of notation, denote any representation of $\theta$ with $\theta$ ). Denote $N_{\alpha \beta}$ for the number of indices ' $u$ ' in the tensor component $T^{\alpha}{ }_{\beta}$ w.r.t. $\mathcal{F}_{u}$. Notice that $N_{\alpha \beta}$ is well-defined: any other $\mathbf{u}$-ONF is related to $\mathcal{F}$ by an $O(n-1)$-spin preserving the number of ' $u$ '-indices. Then, $\theta(T)^{\alpha}{ }_{\beta}$ equals $+T^{\alpha}{ }_{\beta}$ if $N_{\alpha \beta}$ is even, and $-T^{\alpha}{ }_{\beta}$ if $N_{\alpha \beta}$ is odd.

The following properties are immediate from the above definition:

(1) $\theta$ commutes with any tracing $\operatorname{Tr}_{k}$ over $k$ covariant and $k$ contravariant indices of a type $(r, s)$ tensor $T, r, s \geqslant k$ :

$$
\theta\left(\operatorname{Tr}_{k}(T)\right)=\operatorname{Tr}_{k}(\theta(T)) .
$$

(2) For tensors $S \in \mathcal{T}_{s_{1}}^{r_{1}}$ and $T \in \mathcal{T}_{s_{2}}^{r_{2}}$ one has

$$
\theta(S \otimes T)=\theta(S) \otimes \theta(T) .
$$

(3) $\theta$ commutes with lowering or raising indices of a tensor (by contraction with $g_{a b}$ or $g^{a b}$ ), as follows from properties 1 and 2 .

\footnotetext{
${ }^{10}$ If we had taken the special Lorentz group $G=S O(1, n-1)$ instead of the full one, then (A.8) would still give the Cartan involutions for this case, but these do not have an inner action.
} 
A.2.2. Orthogonal splitting. Since $\theta^{2}=1$, we can split the vector space $V$ into \pm 1 eigenspaces, $V=V_{+} \oplus V_{-}$:

$$
V_{+}=\{T \in V \mid \theta(T)=+T\}, \quad V_{-}=\{T \in V \mid \theta(T)=-T\} .
$$

Consequently, for any $T \in V$, we get the split:

$$
T=T_{+}+T_{-}, \quad T_{ \pm}=\frac{1}{2}[T \pm \theta(T)] \in V_{ \pm} .
$$

Thus $\left(T_{+}\right)^{\alpha}{ }_{\beta}=T^{\alpha}{ }_{\beta}$ when $N_{\alpha \beta}$ is even and $\left(T_{+}\right)^{\alpha}{ }_{\beta}=0$ when $N_{\alpha \beta}$ is odd, and vice versa for $T_{-}$(cf supra). In covariant language, $T_{+}\left(T_{-}\right)$is constructed from $T$ by adding all possible contractions with an even (odd) number of $-u^{a} u_{b}$ projectors, completed with $h^{a}{ }_{b}$ projections.

Example A.7. For a rank 3 covariant tensor $T_{a b c}=T_{a[b c]}$ we get

$$
\begin{aligned}
\left(T_{+}\right)_{a b c} & =\left(h_{a}^{d} h_{b}^{e} h_{c}^{f}+u_{a} u^{d} u_{b} u^{e} h_{c}^{f}+u_{a} u^{d} h_{b}^{e} u_{c} u^{f}+h_{a}^{d} u_{b} u^{e} u_{c} u^{f}\right) T_{d e f} \\
& =h_{a}^{d} h_{b}^{e} h_{c}^{f} T_{d e f}+2 u_{a} u_{[b} h_{c]}^{f} T_{u u f} \\
\left(T_{-}\right)_{a b c} & =-\left(h_{a}^{d} h_{b}^{e} u_{c} u^{f}+h_{a}^{d} u_{b} u^{e} h_{c}^{f}+u_{a} u^{d} h_{b}^{e} h_{c}^{f}+u_{a} u^{d} u_{b} u^{e} u_{c} u^{f}\right) T_{d e f} \\
& =-\left(2 h_{a}^{d} h_{[b}^{e} u_{c]} T_{d e u}+u_{a} h_{b}^{e} h_{c}^{f} T_{u e f}\right) .
\end{aligned}
$$

Since $\theta$ is a Lorentz transformation we have $\theta(\boldsymbol{g})=\boldsymbol{g}$, whence $\boldsymbol{g}=\boldsymbol{g}_{+}$. As $\theta$ acts trivially on scalars $f$ we also have $f=f_{+}$. Other immediate properties of this split are the following

(1) Recall that the metric inner product of $S, T \in V$ is defined by

$\boldsymbol{g}(S, T)=g_{a_{1} b_{1}} \cdots g_{a_{r} b_{r}} g^{c_{1} d_{1} \cdots c_{s} d_{s}} S^{a_{1} \cdots a_{r}}{ }_{c_{1} \cdots c_{s}} T^{b_{1} \cdots b_{r}} d_{1} \cdots d_{s}=S^{a_{1} \cdots a_{r}}{ }_{c_{1} \cdots c_{s}} T_{a_{1} \cdots a_{r}}{ }^{c_{1} \cdots c_{s}}$.

Since

$$
\boldsymbol{g}\left(S_{+}, T_{-}\right)=\theta\left(\boldsymbol{g}\left(S_{+}, T_{-}\right)\right)=\theta(\boldsymbol{g})\left(\theta\left(S_{+}\right), \theta\left(T_{-}\right)\right)=-\boldsymbol{g}\left(S_{+}, T_{-}\right)
$$

it follows that the split (A.13) is $\boldsymbol{g}$-orthogonal, $\boldsymbol{g}\left(S_{+}, T_{-}\right)=0$. Hence,

$$
\begin{aligned}
\boldsymbol{g}(S, T) & =\boldsymbol{g}\left(S_{+}, T_{+}\right)+\boldsymbol{g}\left(S_{-}, T_{-}\right) \\
& =\left(\sum_{N_{\alpha \beta}=\text { even }}-\sum_{N_{\alpha \beta}=\text { odd }}\right) S^{\alpha_{1} \alpha_{2} \cdots}{ }_{\beta_{1} \beta_{2} \ldots} T^{\alpha_{1} \alpha_{2} \cdots}{ }_{\beta_{1} \beta_{2} \ldots}
\end{aligned}
$$

(2) From (A.13) and properties 1-3 of $\theta$ it follows that taking the + and - parts of a tensor commutes with any tracing $\operatorname{Tr}_{k}$,

$$
\operatorname{Tr}_{k}(T)_{ \pm}=\operatorname{Tr}_{k}\left(T_{ \pm}\right)
$$

as well as with lowering and raising indices, and that for $S \in \mathcal{T}_{s_{1}}^{r_{1}}$ and $T \in \mathcal{T}_{s_{2}}^{r_{2}}$ we have $(S \otimes T)_{+}=S_{+} \otimes T_{+}+S_{-} \otimes T_{-}$, $(S \otimes T)_{-}=S_{+} \otimes T_{-}+S_{-} \otimes T_{+}$.

As a consequence of (A.20) we get $S=S_{ \pm}, T=T_{ \pm} \Rightarrow S \otimes T=(S \otimes T)_{+}, \quad S=S_{ \pm}, T=T_{\mp} \Rightarrow S \otimes T=(S \otimes T)_{-}$.

In combination with (A.19) and $f=f_{+}$for scalars we thus get in particular:

Proposition A.8. If $T=T_{-}$then also $T^{2 m+1}=\left(T^{2 m+1}\right)_{-}$and $\operatorname{Tr}_{k}\left(T^{2 m+1}\right)=\operatorname{Tr}_{k}\left(T^{2 m+1}\right)_{-}$for any odd power. In particular, if $T$ is a type $(r, r)$ tensor then $\operatorname{Tr}_{(2 m+1) r}\left(T^{2 m+1}\right)=0$. 
A.2.3. Euclidean inner product. The Cartan involution $\theta$ induces an inner product $\langle-,-\rangle$ on $V$ :

$$
\langle S, T\rangle \equiv \boldsymbol{g}(\theta(S), T)=\boldsymbol{g}(S, \theta(T))=\boldsymbol{g}\left(S_{+}, T_{+}\right)-\boldsymbol{g}\left(S_{-}, T_{-}\right) .
$$

In any $\mathbf{u}-\mathrm{ONF}$ we get

$$
\begin{aligned}
& \langle S, T\rangle=\left(\sum_{N_{\alpha \beta}=\text { even }}+\sum_{N_{\alpha \beta}=\text { odd }}\right) S_{\beta_{1} \beta_{2} \ldots T^{\alpha_{1} \alpha_{2} \cdots}{ }_{\beta_{1} \beta_{2} \cdots}^{\alpha_{2} \cdots}} \\
& =\sum_{\boldsymbol{\alpha} \boldsymbol{\beta}} S^{\alpha_{1} \alpha_{2} \cdots}{ }_{\beta_{1} \beta_{2} \ldots} T^{\alpha_{1} \alpha_{2} \cdots}{ }_{\beta_{1} \beta_{2} \ldots} .
\end{aligned}
$$

Compare with (A.17) and (A.18). As is clear from (A.23), $\langle-,-\rangle$ is Euclidean $(\langle T, T\rangle \geqslant$ $0,\langle T, T\rangle=0 \Leftrightarrow T=0)$. Notice that the norm $\|T\|=\langle T, T\rangle^{1 / 2}$ associated to this inner product is $K$-invariant, i.e., for $k \in K$ one has $\|k(T)\|=\|T\|,{ }^{11}$ but it is clearly not invariant under the full Lorentz group.

Remark A.9. The norm $\|T\|$ corresponds to the super-energy density of the tensor $T$ relative to $\mathbf{u}$ (see [10, p 2806], and [78]). Also compare with [2], chapter IX, for the case of Maxwell-like tensors, and with [29, 79] for the Bel-Robinson tensor.

Remark A.10. For later use, we note that if $\mathcal{O}$ is a symmetric (self-adjoint)/ antisymmetric (anti-self-adjoint) linear transformation of $V$ w.r.t. the inner product $g$, i.e., $\boldsymbol{g}(\mathcal{O}(S), T)= \pm \boldsymbol{g}(S, \mathcal{O}(T))$, then $\mathcal{O}_{+}$(resp. $\mathcal{O}_{-}$) is the symmetric/antisymmetric (resp. antisymmetric/symmetric) part of $\mathcal{O}$ w.r.t. the Euclidean inner product $\langle-,-\rangle$. This follows immediately from

$$
\begin{aligned}
\left\langle\left(\mathcal{O}_{+}+\mathcal{O}_{-}\right)(S), T\right\rangle & =\langle\mathcal{O}(S), T\rangle= \pm \boldsymbol{g}(S, \mathcal{O}(\theta(T)))= \pm\langle S, \theta(\mathcal{O})(T)\rangle \\
& = \pm\left\langle S,\left(\mathcal{O}_{+}-\mathcal{O}_{-}\right)(T)\right\rangle
\end{aligned}
$$

\section{A.3. Null alignment theory}

We briefly revise the null alignment theory for tensors over a Lorentzian space developed in [25] (see [80] for a recent review). Let $T \equiv T_{a_{1} \cdots a_{p}}$ be a covariant rank $p$ tensor and $\mathcal{F}_{\ell}=\left\{\mathbf{m}_{\alpha=1, \ldots, n}\right\}=\left\{\mathbf{m}_{0}=\boldsymbol{\ell}, \mathbf{m}_{1}=\mathbf{n}, \mathbf{m}_{\hat{i}=3, \ldots, n}\right\}$ a null frame of $T_{p} M$. Under a positive boost $b_{\lambda}: \quad \ell \mapsto \ell^{\prime}=\mathrm{e}^{\lambda} \boldsymbol{\ell}, \quad \mathbf{n} \mapsto \mathbf{n}^{\prime}=\mathrm{e}^{-\lambda} \mathbf{n}, \quad \mathbf{m}_{\hat{i}} \mapsto \mathbf{m}_{\hat{i}}^{\prime}=\mathbf{m}_{\hat{i}} \quad\left(\lambda \in \mathcal{F}_{M}\right)$

in the $\ell \wedge \mathbf{n}$-plane, the tensor components transform according to

$$
T_{\alpha_{1} \cdots \alpha_{p}} \mapsto T_{\alpha_{1} \cdots \alpha_{p}}^{\prime}=\mathrm{e}^{\lambda b_{\alpha_{1} \cdots \alpha_{p}}} T_{\alpha_{1} \cdots \alpha_{p}}, \quad b_{\alpha_{1} \cdots \alpha_{p}} \equiv \sum_{i=1}^{p}\left(\delta_{\alpha_{i} 0}-\delta_{\alpha_{i} 1}\right),
$$

where $\delta_{\alpha \beta}$ is the Kronecker delta symbol. Thus the integer $b_{\alpha_{1} \cdots \alpha_{p}}$ is the difference between the numbers of 0 - and 1-indices, and is called the b.w. (henceforth abbreviated to b.w.) of the frame component $T_{\alpha_{1} \cdots \alpha_{p}}$ or, rather, of the $p$-tuple $\left(\alpha_{1}, \ldots, \alpha_{p}\right)$. The maximal b.w. of the non-vanishing components of $T$, in its decomposition w.r.t. $\mathcal{F}_{\ell}$, is an invariant of Lorentz

${ }^{11}$ This is an immediate consequence of (A.22), the property (A.7) and the fact that $k$ is a Lorentz transformation. 
transformations preserving the null direction spanned by $\ell$ [25]; it is called the boost order, $b_{T}(\ell)$, of $T$ along $\ell$. Let

$$
b_{\max }(T) \equiv \max _{\{\ell\}} b_{T}(\ell)
$$

denote the maximal value of $b_{T}(\ell)$ taken over all null vectors $\ell$, based on the antisymmetries of $T$. For a generic $\ell$ one has $b_{T}(\ell)=b_{\max }(T)$; if, however, $b_{T}(\ell)<b_{\max }(T)$ then $\ell$ is said to span an AND of alignment order $\mathrm{b}_{\max }(T)-b_{T}(\ell)$. An AND of alignment order $1,2,3, \ldots$ is called single, double, triple, .... Defining

$$
b_{\min }(T) \equiv \min _{\{\ell\}} b_{T}(\ell),
$$

the integer

$$
p_{T} \equiv b_{\max }(T)-b_{\min }(T)
$$

defines the primary alignment type of $T$. Let $\ell$ be a vector of maximal alignment $\left(b_{T}(\ell)=\right.$ $\left.b_{\min }(T)\right)$, then

$$
s_{T} \equiv b_{\max }(T)-\chi_{T}, \quad \chi_{T} \equiv \min _{\left\{\mathbf{n} \mid b_{T}(\boldsymbol{\ell})=b_{\min }(T), n^{a} l_{a}=1\right\}} b_{T}(\mathbf{n})
$$

is the secondary alignment type of $T$, and the couple $\left(p_{T}, s_{T}\right)$ the (full) alignment type.

In agreement with terminology given to the Weyl tensor (see also below), we call a tensor $T$ of type $G$ if it has no ANDs ( $\left.p_{T}=0\right)$ and of type $I$ if it only has one or more ANDs $\left(p_{T} \geqslant 1\right)$. It is of type II or more special if $b_{\min }(T) \leqslant 0\left(p_{T} \geqslant b_{\max }\right)$, i.e., if in a suitable null frame only components of non-positive b.w. are non-vanishing; as a particular case it is of type $D$ if $b_{\min }(T)=\chi_{T}=0\left(p_{T}=s_{T}=b_{\max }\right)$, i.e., only components of zero b.w. are non-vanishing in some null frame $\left\{\boldsymbol{\ell}, \mathbf{n}, \mathbf{m}_{\hat{i}}\right\}$, which is then called canonical. We define $T$ to be of type III if only components of negative b.w. are non-zero (i.e., $\left.p_{T} \geqslant b_{\max }+1\right)$. A further special case occurs when a null vector $\ell$ exists such that $b_{T}(\ell)=-b_{\max }$; then $\ell$ spans the unique AND of $T$ which is thus of type $\left(p_{T}, s_{T}\right)=\left(2 b_{\max }, 0\right)$, also called type $N$. According to these definitions type $\mathrm{N}$ is a subcase of type III, which is a subcase of type II, which is, in turn, a subcase of type I. Of course, for tensors with many indices and few antisymmetries there are a lot of intermediate cases, which may be given specific names if relevant. The trivial case of $T=0$ is dubbed with type $\mathrm{O}$; then one can formally define $b_{T}(\ell):=-b_{\max }-1$ or $b_{T}(\ell):=-\infty$.

The following properties are immediate consequences of the above definitions.

Proposition A.11. Let $\ell$ be a null vector, and $S \neq 0$ and $T \neq 0$ covariant tensors of arbitrary ranks $p$ and $q$, respectively.

- For arbitrary $\alpha, \beta \in \mathcal{F}_{M}$ we have

$$
b_{X}(\ell) \leqslant \max \left(b_{S}(\ell), b_{T}(\ell)\right), \quad X \equiv \alpha S+\beta T .
$$

- For the tensor product of $S$ and $T$,

$$
b_{S \otimes T}(\ell)=b_{S}(\ell)+b_{T}(\ell) .
$$

- If $\operatorname{Tr}_{k}$ is any tracing over $k$ covariant and $k$ contravariant indices $(2 k \leqslant q)$ then

$$
b_{\operatorname{Tr}_{k}(T)}(\ell) \leqslant b_{T}(\ell) \text {. }
$$

- If $\mathbf{n}$ is a second null vector not aligned with $\boldsymbol{\ell}\left(n^{a} l_{a} \neq 0\right)$, then $b_{T}(\ell)+b_{T}(\mathbf{n}) \geqslant 0$. 
By taking $\ell$ and $\mathbf{n}$ maximally aligned $\left(b_{T}(\ell)=b_{\min }(T)\right.$ and $\left.b_{T}(\mathbf{n})=\chi_{T}\right),(\mathrm{A} .31)$ and (A.32) imply:

Corollary A.12. The properties 'type II or more special' and 'type D' are preserved by taking powers of or contractions within a tensor.

Specifically, the Weyl tensor $C_{a b c d}$ of an $n$-dimensional spacetime obeys the Riemann-like symmetries

$$
C_{(a b) c d}=C_{a b(c d)}=0, \quad C_{a b c d}=C_{c d a b}, \quad C_{a[b c d]}=0
$$

and the trace-free property

$$
C^{a}{ }_{\text {bad }}=0 .
$$

In terms of the Riemann and Ricci tensors and the Ricci scalar it is given by

$C_{a b c d}=R_{a b c d}-\frac{2}{n-2}\left(g_{a[c} R_{d] b}-g_{b[c} R_{d] a}\right)+\frac{2 \mathcal{R}}{(n-1)(n-2)} g_{a[c} g_{d] b}$.

For the Riemann, Weyl and Ricci tensors we have $b_{\max }=2$. Let $b_{\text {Ric }}(\ell)$ and $b_{\text {Rie }}(\ell)$ symbolize the boost orders along $\ell$ of the Ricci and Riemann tensor, respectively. Further consequences of proposition A.11 are:

Proposition A.13. For any null vector $\ell$ :

$$
b_{\text {Rie }}(\ell)=\max \left(b_{C}(\ell), b_{\text {Ric }}(\ell) \geqslant b_{C}(\ell), b_{\text {Ric }}(\ell) .\right.
$$

Corollary A.14. The alignment types $\left(p_{R}, s_{R}\right)$ and $\left(p_{C}, s_{C}\right)$ of the Ricci and Weyl tensors at a spacetime point are at least as high as that of the Riemann tensor, i.e., $\max \left(p_{R}, p_{C}\right) \geqslant p_{R}$ and $\max \left(s_{R}, s_{C}\right) \geqslant s_{R}$. In particular, if a Riemann tensor is of type $D$ then the Ricci and Weyl tensors are of type $D$ or $O$ (but not both type $O$ ).

For a non-zero Weyl tensor in particular, an AND is called a WAND. If $p_{C}=0,1,2,3,4$ the primary type has been respectively symbolized by G, I, II, III, N [24, 25]; type O symbolizes a zero Weyl tensor. If $s_{C}=1,2$ this is denoted by $i, i i$ in subscript to the primary symbol. In this paper we will explicitly use or meet types $\mathrm{G}, \mathrm{I}_{i}, \mathrm{II}_{i i} \equiv \mathrm{D}, \mathrm{O}$ and $\mathrm{N}$. In the type $\mathrm{D}$ case, the subtypes $D(a b c)$ and $D(d)$ as described in $[24,15]$ will be relevant, where the former is the conjunction of types $\mathrm{D}(\mathrm{a}), \mathrm{D}(\mathrm{b})$ and $\mathrm{D}(\mathrm{c})$. Here a type D Weyl tensor is said to be of type $\mathrm{D}(\mathrm{abc})(\mathrm{D}(\mathrm{d}))$ if in some Weyl canonical null frame $\left\{\mathbf{m}_{0}=\boldsymbol{\ell}, \mathbf{m}_{1}=\mathbf{n}, \mathbf{m}_{\hat{i}=3, \ldots, n}\right\}$ the components $C_{\hat{i} \hat{j} \hat{k} \hat{l}}\left(C_{01 \hat{i} \hat{j}}\right)$ all vanish (in which case they in fact vanish in any such frame $)^{12}$.

\section{Appendix B. Minimal Ricci- and Maxwell-like tensors}

In example 2.3 we saw that, given any unit timelike vector $\mathbf{u}$, a minimal vector $\mathbf{v}$ is either proportional $(\mathbf{v} \sim \mathbf{u})$ or orthogonal $(\mathbf{v} \perp \mathbf{u})$ to $\mathbf{u}$ and, in particular, cannot be null (or 'type $\mathrm{N}$ '). Conversely, a given vector $\mathbf{v}$ is minimal w.r.t. the unit vector parallel to it if $\mathbf{v}$ is timelike (or 'type G'), and w.r.t. any $\mathbf{u} \perp \mathbf{v}$ when $\mathbf{v}$ is spacelike (or 'type D'). This provides an explicit proof for proposition 2.7 in the case of vectors. Likewise, we give here more explicit proofs in the case of Ricci- and Maxwell-like rank 2 tensors.

\footnotetext{
${ }^{12}$ It is easy to show that the Lorentz transformations which convert a Weyl canonical null frame into another one subjects the separate component sets $\left[C_{\hat{i} \hat{j} \hat{k} \hat{l}}\right]$ and $\left[C_{01 \hat{i} \hat{j}}\right]$ to an invertible transformation. Hence the vanishing of such a set is a well-defined property. The same holds regarding the separate subtypes $\mathrm{D}(\mathrm{a}), \mathrm{D}(\mathrm{b})$ and $\mathrm{D}(\mathrm{c})$.
} 


\section{B.1. Ricci-like tensors}

Let $\left(V_{n}, \boldsymbol{g}\right)$ be a vector space of arbitrary dimension $n$, equipped with a (non-degenerate) metric $\boldsymbol{g}$ of arbitrary signature $s$. Petrov [81] deduced canonical forms for Ricci-like tensors $R_{a b}=R_{(a b)}$ over $\left(V_{n}, \boldsymbol{g}\right)$, connected to the Jordan canonical forms of $R_{b}^{a} \equiv g^{a c} R_{c b}$. For Lorentzian signature $s=n-2$ there are four distinct possibilities (see also [82, 83]), where the Segre types (but not possible eigenvalue degeneracies) are indicated between brackets:

Type R1 $(\{1,1 \cdots 1\}): R_{a b}=\rho_{u} u_{a} u_{b}+\sum_{i=2}^{n} \rho_{i} m_{a}^{i} m_{b}^{i}$;

Type R2 $\quad(\{z \bar{z} 1 \cdots 1\}): R_{a b}=2 \alpha u_{(a} m_{b)}^{2}+\beta\left(u_{a} u_{b}-m_{a}^{2} m_{b}^{2}\right)+\sum_{\hat{i}=3}^{n} \rho_{i} m_{a}^{\hat{i}} m_{b}^{\hat{i}}, \quad \alpha \neq 0$

Type R3 $(\{21 \cdots 1\}): R_{a b}=2 \alpha l_{(a} n_{b)} \pm l_{a} l_{b}+\sum_{\hat{i}=3}^{n} \rho_{i} m_{a}^{\hat{i}} m_{b}^{\hat{i}}$

Type R4 $(\{31 \cdots 1\}): R_{a b}=\alpha\left(2 l_{(a} n_{b)}+m_{a}^{3} m_{b}^{3}\right)+2 l_{(a} m_{b)}^{3}+\sum_{\hat{i}=4}^{n} \rho_{i} m_{a}^{\hat{i}} m_{b}^{\hat{i}}$,

where, as usual, $\ell$ and $\mathbf{n}$ are null, $\mathbf{u}$ is unit timelike and the $\mathbf{m}^{i}\left(\mathbf{m}^{\hat{i}}\right)$ are unit spacelike. For our purposes it is enough to mention that:

(1) Types R3 and R4 have, while types R1 and R2 do not have, a unique null eigendirection (spanned by $l^{a}$ ). But for a symmetric tensor, null eigendirections are precisely ANDs of boost order $\leqslant 0$ (since the equation $l_{[a} R_{b] c} l^{c}=0$ expresses both conditions at the same time). Hence, types $R 3$ and $R 4$ precisely cover the alignment types 'II or more special, but not $D$ nor $O$ '. Type $\mathrm{R} 1$ comprises the alignment types $\mathrm{O}$ and $\mathrm{D}$ (without loss of generality for $\rho_{2}=-\rho_{u}$, see also the proof of proposition 4.11), while types I and G are distributed over types R1 and R2, where type I implies type $\mathrm{I}_{i}$ and at least a $(n-3)$-dimensional surface of single ANDs (see proposition 4.10). As an example, the Ricci tensor given in equation (89) is of type R1 and of alignment type $I_{i}$.

(2) Type R1 is the only type having one or more timelike eigendirections (one of them spanned by $u^{a}$ ). Type $\mathrm{R} 2$ has two complex eigenvectors $\mathbf{u} \pm \mathbf{i m}_{2}$ corresponding to the eigenvalues $-\beta \pm \mathrm{i} \alpha$. In the adapted canonical null frame $\mathcal{F}_{c}=\left\{\mathbf{m}_{0}=\boldsymbol{\ell}, \mathbf{m}_{1}=\mathbf{n}, \mathbf{m}_{\hat{i}}\right\}$, where $\boldsymbol{\ell}$ and $\mathbf{n}$ are defined by (1), the R2 canonical form becomes

$$
R_{a b}=\alpha\left(l_{a} l_{b}-n_{a} n_{b}\right)-2 \beta l_{(a} n_{b)}+\sum_{\hat{i}=3}^{n} \rho_{i} m_{a}^{\hat{i}} m_{b}^{\hat{i}}, \quad \alpha \neq 0 .
$$

In view of point 1 we need to show that equation (8) admits a solution precisely for types $\mathrm{R} 1$ and R2. In a $\mathbf{u}-\mathrm{ONF}\left\{\mathbf{u}, \mathbf{m}_{2}, \mathbf{m}_{\hat{i}}\right\}$, where the vector $\mathbf{m}_{2}$ has been isolated, (8) splits into

$$
R_{2 u}\left(R_{u u}+R_{22}\right)+R_{u \hat{j}} R_{2}{ }^{\hat{j}}=0, \quad R_{u u} R_{u \hat{i}}+R_{u 2} R_{2 \hat{i}}+R_{u \hat{j}} R_{\hat{i}}^{\hat{j}}=0 .
$$

- In type R1 there is at least one eigenvector $\mathbf{u}$, which satisfies $R_{i u}=0, \forall i$, and thus (8).

- For type R2 we take the $\mathbf{u}-\mathrm{ONF}\left\{\mathbf{u}, \mathbf{m}_{2}, \mathbf{m}_{\hat{i}}\right\}$ from the canonical form. Then $R_{u u}=-R_{22}=$ $\alpha$ and $R_{u 2}=R_{u \hat{i}}=R_{2 \hat{i}}=0$, such that equation (B.2) is satisfied and $R_{a b}$ is minimal w.r.t. u.

- In any null frame $\left\{\mathbf{m}_{0}=\boldsymbol{\ell}, \mathbf{m}_{1}=\mathbf{n}, \mathbf{m}_{\hat{i}=3, \ldots, n}\right\}$ adapted to $\left\{\mathbf{u}, \mathbf{m}_{2}, \mathbf{m}_{\hat{i}=3, \ldots, n}\right\}$, the first equation of (B.2) becomes

$$
R_{00}^{2}+\sum_{\hat{i}=3}^{n} R_{0 \hat{i}}^{2}=R_{11}^{2}+\sum_{\hat{i}=3}^{n} R_{1 \hat{i}}^{2} .
$$


We see that if $R_{a b}$ is minimal w.r.t. a certain $\mathbf{u}$ and has an AND of boost order $\leqslant 0$ spanned by $\ell$ (i.e., $R_{00}=R_{0 \hat{i}}=0, \forall \hat{i}$ ), then the vector $\mathbf{n}$ defined by (36) necessarily spans an AND of boost order $\leqslant 0$ as well. By point 1 this excludes types R3 and R4, for which there is only one double AND (spanned by $\ell$ in their canonical forms).

This shows that Ricci-like tensors of types R1 and R2 (alignment types G, I, D and O) are minimal w.r.t. a certain unit timelike vector $\mathbf{u}$, whereas those of types R3 and R4 (alignment types II (not D), III or N) are not.

We observe also that type R1 is precisely the case of a PE Ricci tensor, while type R2 contains the purely magnetic case where we can take $\beta=0=\rho_{i}$, cf proposition 4.4 and remark 4.5.

\section{B.2. Maxwell-like tensors}

Maxwell-like tensors $F_{a b}=F_{[a b]}$ have $b_{\max }=1$ and can be of alignment types G, D, O, II and $\mathrm{N}$ (we assume hereafter $n>2$ since any non-zero bivector is trivially of type $\mathrm{D}$ in two dimensions). Type $\mathrm{G}$ (no AND) can only occur when $n$ is odd [84, 85] (see also remark B.2 below). Type $\mathrm{O}$ is the trivial case $F_{a b}=0$. Types II and $\mathrm{N}$ allow for precisely one AND (of boost order 0 and -1 , respectively); in four [9, 82] and higher [86] dimensions the $F_{a b}$ of type $\mathrm{N}$ are null Maxwell-like tensors in the sense that all polynomial invariants vanish. For type $\mathrm{D}$ there are two or more ANDs.

Let $F_{a b} \neq 0$ and consider the symmetric tensor $\left(F^{2}\right)_{a b} \equiv F_{a c} F^{c}{ }_{b}$. In view of the minimal criterion (9) for $F_{a b}$, we need to show that $\left(F^{2}\right)^{a}{ }_{b}$ has a timelike eigenvector iff $F_{a b}$ has no unique AND (i.e., it is not of type II or N). This will follow immediately from:

Proposition B.1. The symmetric tensor $\left(F^{2}\right)_{a b}$ is of type R1 or R3. $\ell$ is an AND of $F_{a b} \neq 0$ iff it is an AND for $\left(F^{2}\right)_{a b}$ of boost order $\leqslant 0$.

Proof. Take an arbitrary null frame $\mathcal{F}=\left\{\mathbf{m}_{\alpha}\right\}=\left\{\mathbf{m}_{0}=\boldsymbol{\ell}, \mathbf{m}_{1}=\mathbf{n}, \mathbf{m}_{\hat{i}}\right\}$. Then

$$
\left(F^{2}\right)_{1 \alpha}=F_{1 a} F_{\alpha}^{a}=F_{10} F_{1 \alpha}+F_{1 \hat{j}} F_{\alpha}^{\hat{j}}, \quad\left(F^{2}\right)_{0 \beta}=F_{0 a} F_{\beta}^{a}=F_{01} F_{0 \beta}+F_{0 \hat{j}} F_{\beta}^{\hat{j}} .
$$

When applied to $\alpha=1, \beta=0$ and $\beta=\hat{i}$ this gives

$$
\left(F^{2}\right)_{11}=-\sum_{\hat{j}=3}^{n} F_{1 \hat{j}}^{2} \leqslant 0, \quad\left(F^{2}\right)_{00}=-\sum_{\hat{j}=3}^{n} F_{0 \hat{j}}^{2} \leqslant 0, \quad\left(F^{2}\right)_{0 \hat{i}}=F_{01} F_{0 \hat{i}}+F_{0 \hat{j}} F^{\hat{j}}{ }_{\hat{i}} .
$$

It follows from the last two equations that $F_{0 \hat{i}}=0, \forall \hat{i} \Leftrightarrow\left(F^{2}\right)_{00}=\left(F^{2}\right)_{0 \hat{i}}=0, \forall \hat{i}$, which proves the second statement. As for the first statement, suppose now that $R_{a b}=\left(F^{2}\right)_{a b}$ were of type R2 and take the null canonical form (B.1) associated to the canonical null frame $\mathcal{F}_{c}$. We would have $\left(F^{2}\right)_{11}=-\left(F^{2}\right)_{00}=\alpha \neq 0$, whence $\left(F^{2}\right)_{00}\left(F^{2}\right)_{11}<0$, in contradiction with the first two equations of (B.4). Finally, suppose that $R_{a b}=\left(F^{2}\right)_{a b}$ were of type R4. In the canonical null frame associated to the canonical form we have, in particular, $\left(F^{2}\right)_{13}=1$ and $\left(F^{2}\right)_{11}=0$. From the latter equation and the first equation in (B.4) we get $F_{1 \hat{i}}=0, \forall \hat{i}$, but the first equation of (B.3), with $\alpha=3$, then leads to the contradiction $\left(F^{2}\right)_{13}=0$.

From this proposition and points 1 and 2 in section B. 1 we conclude: if $\left(F^{2}\right)_{a b}$ is of type $\mathrm{R} 1$ it possesses a timelike eigenvector and not a unique AND of boost order $\leqslant 0$, i.e., $F_{a b}$ does not have a unique AND; if $\left(F^{2}\right)_{a b}$ is of type R3 it possesses no timelike eigenvector but does have a unique AND of boost order $\leqslant 0$, i.e., $F_{a b}$ has a unique AND. It follows that $F_{a b}$ is minimal w.r.t. a certain $\mathbf{u}$ iff it does not possess a unique AND, which is the case iff $\left(F^{2}\right)_{a b}$ is of type R1. 
Remark B.2. In fact, these results can be shown more directly by considering the classification of Maxwell-like tensors $F_{a b}$ into three different types and their corresponding canonical forms. We also indicate the Segre type; degeneracy of the eigenvalue 0 is indicated by round brackets, but additional degeneracies may occur in the $z \bar{z}$ parts.

Type F1 $\quad(\{(1,1 \cdots 1) z \bar{z} \cdots z \bar{z}\}): \quad F_{a b}=\sum_{k=1}^{r} 2 f_{k} v_{[a}^{2 k-1} v_{b]}^{2 k}, \quad f_{k} \neq 0$;

Type F2 $(\{11(1 \cdots 1) z \bar{z} \cdots z \bar{z}\}): \quad F_{a b}=\sum_{k=1}^{r} 2 f_{k} v_{[a}^{2 k-1} v_{b]}^{2 k}+2 \sigma l_{[a} n_{b]}, \quad f_{k} \neq 0 \neq \sigma ;$

Type F3 $(\{(31 \cdots 1) z \bar{z} \cdots z \bar{z}\}): \quad F_{a b}=\sum_{k=1}^{r} 2 f_{k} v_{[a}^{2 k-1} v_{b]}^{2 k}+2 l_{[a} v_{b]}^{2 r+1}, \quad f_{k} \neq 0$.

Here $r \leqslant\left\lfloor\frac{n-\mathrm{i}}{2}\right\rfloor$ for type Fi. The vectors $\ell, \mathbf{n}$ and $\mathbf{v}^{l}$ are part of a null frame $(\boldsymbol{\ell}$ and $\mathbf{n}$ being real null and the $\mathbf{v}^{l}$ unit spacelike). A scalar $f_{k}$ corresponds to a complex conjugate pair of eigenvalues $\pm \mathrm{i} f_{k}$, with complex null eigenvectors $v^{2 k-1} \pm \mathrm{i} v^{2 k}$ and the corresponding elementary divisors being linear. Analogously as for the Ricci-like (symmetric) case, this classification can be easily derived based on the antisymmetry of $F_{a b}$ and the fact that for Lorentzian signature orthogonal null vectors are parallel; see also [85]. The possible numbers of independent (real) null eigendirections (ANDs) were discussed in [84], pp 5313; notice that a null vector $v^{b}$ is an eigenvector of $F_{b}^{a}$ iff it is an AND (joint condition $v_{[a} F_{b] c} v^{c}=0$ ); hence, in particular, all null vectors of the kernel span ANDs.

- Type F1 tensors $F_{a b}$ are precisely the purely magnetic ones $\left(F=F_{+}\right.$w.r.t. a certain $\left.\mathbf{u}\right)$. The null alignment type is $\mathrm{G}$ if and only if $n$ is odd and $r=(n-1) / 2$; in this case the (onedimensional) kernel is spanned by a unique unit timelike vector $\mathbf{u}$ w.r.t. which $F=F_{+}$. In all other cases the alignment type is $\mathrm{D}$ (or $\mathrm{O}$, corresponding to $r=0$ ), the ANDs and the $\mathbf{u}$ spanning precisely the null and timelike directions of the kernel (in accordance with remark 3.14). In any case $\mathbf{u}$ belongs to the kernel of $\left(F^{2}\right)^{a}{ }_{b}$ (which is type R1) and thus $F_{a b}$ is minimal w.r.t. u. Notice that type $\mathrm{G}$ cannot occur in cases F2 and F3 below, so that all type $\mathrm{G}$ tensors $F_{a b}$ are necessarily PM.

- Type F2 tensors are all of alignment type D. There are precisely two (real) ANDs, spanned by $\boldsymbol{\ell}$ and $\mathbf{n}$ and corresponding to the real eigenvalues $+\sigma$ and $-\sigma$, respectively. We have $F=F_{-}$iff $r=0$ (this is automatically true when $n=3$ ). If $n \geqslant 4$ and when there is at least one pair of imaginary eigenvalues $\pm \mathrm{i} f_{k}$ this gives (the only) examples of minimal Maxwell-like tensors for which $F_{+} \neq F \neq F_{-}$. In any case the $\boldsymbol{\ell} \wedge \mathbf{n}$ plane is a timelike eigenplane of $\left(F^{2}\right)^{a}{ }_{b}$ (which is type R1) such that $F_{a b}$ is minimal w.r.t. any unit timelike $\mathbf{u}$ in this plane.

- For type F3 tensors $F_{a b}, \boldsymbol{\ell}$ spans the unique AND (corresponding to a cubic elementary divisor $x^{3}$ ). Thus $F_{a b}$ is of type F3 iff it is of alignment type II or (when $r=0$ ) N. The Ricci-like tensor $\left(F^{2}\right)_{a b}$ is of type R3 (with, in particular, $\alpha=0$ in the corresponding canonical form); thus it has no timelike eigenvectors and cannot be minimal w.r.t. a unit timelike $\mathbf{u}$.

\section{Appendix C. Timelike unit vector fields: expansion, rotation, shear, and Raychaudhuri equation}

We consider a timelike unit vector field $\mathbf{u}, u_{a} u^{a}=-1$, and follow the notation of chapter 6 of [9]. The purpose here is to write parts of the Riemann and Weyl tensors in terms of 
the kinematic quantities of $\mathbf{u}$, as defined in (C.1)-(C.5) below (see [4] for a comprehensive overview of results in four dimensions). We first define the projector

$$
h_{a b}=g_{a b}+u_{a} u_{b},
$$

such that $h_{a b} u^{b}=0$. This enables us to define the rotation, expansion and shear tensors as

$$
\omega_{a b}=h_{a}^{c} h_{b}^{d} u_{[c ; d]}, \quad \Theta_{a b}=h_{a}^{c} h_{b}^{d} u_{(c ; d)}, \quad \sigma_{a b}=\Theta_{a b}-\tilde{\Theta} h_{a b},
$$

where $\tilde{\Theta}$ is a normalized (volume) expansion scalar defined by

$$
(n-1) \tilde{\Theta}=\Theta \equiv h^{a b} \Theta_{a b}=u_{; a}^{a},
$$

and the acceleration vector

$$
\dot{u}_{a}=u_{a ; b} u^{b} .
$$

The tensors (C.2) and (C.4) are all spatial, i.e., $\omega_{a b} u^{a}=\Theta_{a b} u^{a}=\sigma_{a b} u^{a}=\dot{u}_{a} u^{a}=0$. One can write the covariant derivative of $\mathbf{u}$ in the standard way, namely

$$
u_{a, b}=-\dot{u}_{a} u_{b}+\omega_{a b}+\sigma_{a b}+\tilde{\Theta} h_{a b} .
$$

Using this, the Ricci identity $2 u_{a ;[b c]}=R_{a b c}^{d} u_{d}$ becomes

$$
\begin{aligned}
\frac{1}{2} R_{a b c}^{d} u_{d}= & -\dot{u}_{a ;[c} u_{b]}+\left(-\dot{u}_{a}+\tilde{\Theta} u_{a}\right)\left(-\dot{u}_{[b} u_{c]}+\omega_{b c}\right)+\omega_{a[b ; c]}+\sigma_{a[b ; c]}+h_{a[b} h_{c]}{ }^{d} \tilde{\Theta}_{, d} \\
& +\tilde{\Theta}\left(\omega_{a[c}+\sigma_{a[c}\right) u_{b]}+\left(\dot{\tilde{\Theta}}+\tilde{\Theta}^{2}\right) h_{a[c} u_{b]},
\end{aligned}
$$

By contraction this gives

$R_{b}^{d} u_{d}=-\dot{u}_{; a}^{a} u_{b}+\dot{u}^{a}\left(\omega_{a b}-\sigma_{a b}\right)+\omega_{b ; a}^{a}+\sigma_{b ; a}^{a}-(n-2) h_{b}{ }^{c} \tilde{\Theta}_{, c}+(n-1)\left(\dot{\tilde{\Theta}}+\tilde{\Theta}^{2}\right) u_{b}$,

where a dot denotes a derivative along $\mathbf{u}$.

We now multiply (C.6) by $u^{b}$. The symmetric part of the resulting equation can be written as

$R_{a b c}^{d} u_{d} u^{b}=\dot{u}_{a} \dot{u}_{c}-\omega_{a b} \omega_{c}^{b}-\sigma_{a b} \sigma_{c}^{b}-2 \tilde{\Theta} \sigma_{a c}-\left(\dot{\tilde{\Theta}}+\tilde{\Theta}^{2}\right) h_{a c}+h_{a}^{d} h_{c}^{e}\left(\dot{u}_{(d ; e)}-\dot{\sigma}_{d e}\right)$,

where we used the identities $h_{a}^{d} h_{c}^{e} \dot{u}_{(d ; e)}=h_{(c}^{b} \dot{u}_{a) ; b}+\dot{u}^{b} u_{(c}\left(\omega_{a) b}-\sigma_{a) b}\right)-\tilde{\Theta} \dot{u}_{(a} u_{c)}$ and $h_{a}^{d} h_{c}^{e} \dot{\sigma}_{d e}=\dot{\sigma}_{a c}+2 u^{b} u_{(c} \dot{\sigma}_{a) b}$, while the antisymmetric part reads

$$
h_{a}^{d} h_{c}^{e} \dot{\omega}_{d e}=2 \sigma_{[a}^{b} \omega_{c] b}-2 \tilde{\Theta} \omega_{a c}+h_{a}^{d} h_{c}^{e} \dot{u}_{[d ; e]},
$$

in which the identities $h_{a}^{d} h_{c}^{e} \dot{u}_{[d ; e]}=h_{[c}^{b} \dot{u}_{a] ; b}-\dot{u}^{b}\left(\omega_{b[c}+\sigma_{b[c}\right) u_{a]}+\tilde{\Theta} \dot{u}_{[a} u_{c]}$ and $h_{a}^{d} h_{c}^{e} \dot{\omega}_{d e}=$ $\dot{\omega}_{a c}+2 \dot{u}^{b} \omega_{b[a} u_{c]}$ have been employed.

Further, the trace of (C.8) gives the Raychaudhuri equation

$$
R_{b}^{d} u_{d} u^{b}=\dot{u}_{; d}^{d}+\omega_{a b} \omega^{a b}-\sigma_{a b} \sigma^{a b}-(n-1)\left(\dot{\tilde{\Theta}}+\tilde{\Theta}^{2}\right) .
$$

Substituting in (C.8) the standard definition of the Weyl tensor and using (C.10) and the identities $h_{a}^{d} h_{c}^{e} R_{d e}=R_{a c}+2 u_{(a} R_{c) u}+u_{a} u_{c} R_{u u}$ and $h^{d e} R_{d e}=R+R_{u u}$, we can write the (electric) components $C_{a b c}^{d} u_{d} u^{b}$ of the Weyl tensor as

$$
\begin{aligned}
C_{a b c}^{d} u_{d} u^{b}= & \dot{u}_{a} \dot{u}_{c}-\omega_{a b} \omega_{c}^{b}-\sigma_{a b} \sigma_{c}^{b}-2 \tilde{\Theta} \sigma_{a c}+h_{a}^{d} h_{c}^{e}\left(\dot{u}_{(d ; e)}-\dot{\sigma}_{d e}+\frac{R_{d e}}{n-2}\right) \\
& -h_{a c} \frac{1}{n-1}\left(\dot{u}_{; d}^{d}+\omega_{d e} \omega^{d e}-\sigma_{d e} \sigma^{d e}+\frac{h^{d e} R_{d e}}{n-2}\right) .
\end{aligned}
$$


The magnetic components can be expressed in terms of

$$
\begin{aligned}
C_{b c}^{d g} u_{d} h_{e}^{b} h_{f}^{c}= & 2 h^{a g} h_{e}^{b} h_{f}^{c}\left(-\dot{u}_{a} \omega_{b c}+\omega_{a[b ; c]}+\sigma_{a[b ; c]}\right) \\
& +\frac{2}{n-2} h^{g}{ }_{[e}\left[\left(\omega_{f]}^{a}-\sigma_{f]}^{a}\right) \dot{u}_{a}+h_{f]}^{b}\left(\omega_{b ; a}^{a}+\sigma_{b ; a}^{a}\right)\right] .
\end{aligned}
$$

The above equations reduce to formulae (6.26)-(6.30) in [9] when $n=4 .{ }^{13}$ Remember, however, that the electric part of the Weyl tensor consists also of $C_{i j k l}$, which is not described by (C.11) for $n \geqslant 5$. Note that in the special case of a geodesic $\mathbf{u}$ one has $\dot{\mathbf{u}}=0$ and the above equations get a simpler form, cf, e.g., [88].

\section{References}

[1] Hervik S 2011 A spacetime not characterized by its invariants is of aligned type II Class. Quantum Grav. 28215009

[2] Synge J L 1955 Relativity: The Special Theory (Amsterdam: North-Holland)

[3] Matte A 1953 Sur de nouvelles solutions oscillatoires de équations de la gravitation Can. J. Math. 5 1-16

[4] Ellis G F R 1971 Relativistic cosmology Proceedings of the International School of Physics 'Enrico Fermi', Course 47: General Relativity and Cosmology vol 16 ed R K Sachs (New York: Academic) pp 104-82 Reprinted in Ellis G F R 2009 Gen. Rel. Grav. 41 581-600

[5] McIntosh C B G, Arianrhod R, Wade S T and Hoenselaers C 1994 Electric and magnetic Weyl tensors: classification and analysis Class. Quantum Grav. 11 1555-64

[6] Geroch R 1977 Asymptotic structure of space-time Asymptotic Structure of Space-time ed F P Esposito and L Witten (New York: Plenum) pp 1-105

[7] Ashtekar A and Hansen R O 1978 A unified treatment of null and spatial infinity in general relativity: I. Universal structure, asymptotic symmetries, and conserved quantities at spatial infinity J. Math. Phys. 19 1542-66

[8] Ashtekar A 1980 Asymptotic structure of gravitational field at spatial infinity General Relativity and Gravitation: One Hundred Years After the Birth of Albert Einstein vol 2 ed A Held (New York: Plenum) pp 37-69

[9] Stephani H, Kramer D, MacCallum M, Hoenselaers C and Herlt E 2003 Exact Solutions of Einstein's Field Equations 2nd edn (Cambridge: Cambridge University Press)

[10] Senovilla J M M 2000 Super-energy tensors Class. Quantum Grav. 17 2799-841

[11] Senovilla J M M 2001 General electric-magnetic decomposition of fields, positivity and Rainich-like conditions Reference Frames and Gravitomagnetism ed J F Pascual-Sánchez, L Floría, A San Miguel and F Vicente (Singapore: World Scientific) pp 145-64

[12] Mann R B and Marolf D 2006 Holographic renormalization of asymptotically flat spacetimes Class. Quantum Grav. $232927-50$

[13] Tanabe K, Tanahashi N and Shiromizu T 2009 Asymptotic flatness at spatial infinity in higher dimensions J. Math. Phys. 50072502

[14] Richardson R W and Slodowy P 1990 Minimum vectors for real reductive algebraic groups J. Lond. Math. Soc. 42 409-29

[15] Ortaggio M 2009 Bel-Debever criteria for the classification of the Weyl tensor in higher dimensions Class. Quantum Grav. 26195015

[16] Coley A and Hervik S 2010 Higher dimensional bivectors and classification of the Weyl operator Class. Quantum Grav. 27015002

[17] Wylleman L and Van den Bergh N 2006 Complete classification of purely magnetic, nonrotating, nonaccelerating perfect fluids Phys. Rev. D 74084001

[18] Arianrhod R and McIntosh C B G 1992 Principal null directions of Petrov type I Weyl spinors: geometry and symmetry Class. Quantum Grav. 9 1969-82

[19] Coley A, Hervik S, Ortaggio M and Wylleman L 2012 Refinements of the Weyl tensor classification in five dimensions Class. Quantum Grav. 29155016

[20] Macdonald I G 1979 Symmetric Functions and Hall Polynomials (Oxford: Clarendon)

[21] Coley A and Hervik S 2011 Discriminating the Weyl type in higher dimensions using scalar curvature invariants Gen. Rel. Grav. 43 2199-207

[22] Trümper M 1965 On a special class of type-I gravitational fields J. Math. Phys. 6 584-9

${ }^{13}$ Formula (C.9) is not displayed in [9], but agrees with (4.22) of [87]. However, in equation (4.27) of this last standard reference the $\dot{u}_{a} \dot{u}_{c}$ term appearing in (C.11), necessary for tracelessness since $h^{d e} \dot{u}_{(d ; e)}=\dot{u}_{; d}^{d}-\dot{u}^{d} \dot{u}_{d}$, is missing. 
[23] Haddow B M 1995 Purely magnetic space-times J. Math. Phys. 36 5848-54

[24] Coley A, Milson R, Pravda V and Pravdová A 2004 Classification of the Weyl tensor in higher dimensions Class. Quantum Grav. 21 L35-L41

[25] Milson R, Coley A, Pravda V and Pravdová A 2005 Alignment and algebraically special tensors in Lorentzian geometry Int. J. Geom. Methods Mod. Phys. 2 41-61

[26] Lozanovski C and Carminati J 2002 Purely magnetic locally rotationally symmetric spacetimes Gen. Rel. Grav. 34 853-63

[27] Van den Bergh N and Wylleman L 2006 An exhaustive classification of aligned Petrov type D purely magnetic perfect fluids Class. Quantum Grav. $233353-9$

[28] Godazgar M and Reall H S 2012 Peeling of the Weyl tensor and gravitational radiation in higher dimensions Phys. Rev. D 85084021

[29] Bel L 1962 Les états de radiation et le problème de l'énergie en relativité générale Cah. Phys. 16 59-80 Reprinted in Bel L 2000 Gen. Rel. Grav. 322047

[30] Barnes A 2004 Purely magnetic spacetimes Proc. 27th Spanish Relativity Meeting (Alicante, Spain, Sept. 2003) ed J Miralles, J Font and J Pons (Alicante: University of Alicante Press) arXiv:gr-qc/0401068

[31] Godazgar M and Reall H S 2009 Algebraically special axisymmetric solutions of the higher-dimensional vacuum Einstein equation Class. Quantum Grav. 26165009

[32] Durkee M 2009 Type II Einstein spacetimes in higher dimensions Class. Quantum Grav. 26195010

[33] Durkee M and Reall H S 2009 A higher-dimensional generalization of the geodesic part of the Goldberg-Sachs theorem Class. Quantum Grav. 26245005

[34] Ortaggio M, Pravda V and Pravdová A 2011 On higher-dimensional Einstein spacetimes with a warped extra dimension Class. Quantum Grav. 28105006

[35] Wylleman L On Weyl type II or more special spacetimes in higher dimensions, in preparation

[36] Wylleman L Notes on the null alignment classification of spacetime tensors, in preparation

[37] Pravda V and Pravdová A 2005 WANDs of the black ring Gen. Rel. Grav. 37 1277-87

[38] Narain U 1970 Some properties of gravitational fields of magnetic type Phys. Rev. D 2 278-80

[39] Trümper M 1962 Zur Bewegung von Probekörpern in Einsteinschen Gravitations-Vakuumfeldern Z. Phys. $16855-60$

[40] Wylleman L and Beke D 2010 Expanding perfect fluid generalizations of the C metric Phys. Rev. D 81104038

[41] Barnes A 1973 On Birkhoff's theorem in general relativity Commun. Math. Phys. 33 75-82

[42] Wylleman L 2008 A Petrov-type I and generically asymmetric rotating dust family Class. Quantum Grav. 25172001

[43] Pravda V, Pravdová A and Ortaggio M 2007 Type D Einstein spacetimes in higher dimensions Class. Quantum Grav. 24 4407-28

[44] Ramos M P M, Vaz E G L R and Carot J 2003 Double warped space-times J. Math. Phys. 44 4839-65

[45] Brinkmann H W 1925 Einstein spaces which are mapped conformally on each other Math. Ann. 94 119-45

[46] Pravda V, Pravdová A, Coley A and Milson R 2004 Bianchi identities in higher dimensions Class. Quantum Grav. $212873-97$

See also Pravda V, Pravdová A, Coley A and Milson R 2007 Class. Quantum Grav. 241691 (corrigendum)

[47] Ehlers J and Kundt W 1962 Exact solutions of the gravitational field equations Gravitation: An Introduction to Current Research ed L Witten (New York: Wiley) pp 49-101

[48] Collins C B 1984 Shear-free perfect fluids with zero magnetic Weyl tensor J. Math. Phys. 25 995-1000

[49] Sklavenites D 1985 Relativistic, stationary, axisymmetric perfect fluids: II. Solutions with vanishing magnetic Weyl tensor J. Math. Phys. 26 2279-81

[50] Senovilla J M M 1987 On Petrov type-D stationary axisymmetric rigidly rotating perfect-fluid metrics Class. Quantum Grav. 4 L115-L119

[51] Senovilla J M M 1992 New family of stationary and axisymmetric perfect-fluid solutions Class. Quantum Grav. 9 L167-L169

[52] Arianrhod R, Lun A W-C, McIntosh C B G and Perjés Z 1994 Magnetic curvatures Class. Quantum Grav. $112331-5$

[53] Stewart J M and Ellis G F R 1968 Solutions of Einstein's equations for a fluid which exhibits local rotational symmetry J. Math. Phys. 9 1072-82

[54] Lozanovski C and Carminati J 2003 Purely magnetic locally rotationally symmetric spacetimes Class. Quantum Grav. 20 215-38

[55] Bonnor W B 1995 The magnetic Weyl tensor and the van Stockum solution Class. Quantum Grav. 12 1483-9

[56] Myers R C and Perry M J 1986 Black holes in higher-dimensional space-times Ann. Phys., NY 172 304-47

[57] Ortaggio M, Pravda V and Pravdová A 2009 Higher dimensional Kerr-Schild spacetimes Class. Quantum Grav. 26025008 
[58] Emparan R and Reall H S 2002 A rotating black ring solution in five dimensions Phys. Rev. Lett. 88101101

[59] Yau S T 1973 Remarks on conformal transformations J. Diff. Geom. 8 369-81

[60] Ficken F A 1939 The Riemannian and affine differential geometry of product-spaces Ann. Math. 40 892-913

[61] Goodman R and Wallach N R 2009 Symmetry, Representations and Invariants (New York: Springer)

[62] Milnor J 1976 Curvatures of left invariant metrics on Lie groups Adv. Math. 21 293-329

[63] Hervik S 2010 Negatively curved left-invariant metrics on Lie groups arXiv:1002.2106

[64] Ellis G and MacCallum M A 1969 A class of homogeneous cosmological models Commun. Math. Phys. 12 108-41

[65] Hervik S 2002 Multidimensional cosmology: spatially homogeneous models of dimension $(4+1)$ Class. Quantum Grav. 19 5409-28

[66] Hervik S 2001 Discrete symmetries in translation invariant cosmological models Gen. Rel. Grav. 33 2027-48

[67] Lozanovski C 2007 Szekeres-type mappings of Kasner and Petrov type $I\left(M^{+}\right)$purely magnetic spacetimes Class. Quantum Grav. 24 1169-88

[68] Lozanovski C and Wylleman L 2011 Complex windmill transformation producing new purely magnetic fluids Class. Quantum Grav. 28075015

[69] Wylleman L 2006 Anti-Newtonian universes do not exist Class. Quantum Grav. 23 2727-40

[70] Hall G 1973 On the Petrov classification of gravitational fields J. Phys. A: Math. Nucl. Gen. 6 619-23

[71] Durkee M, Pravda V, Provdová A and Reall H S 2010 Generalization of the Geroch-Held-Penrose formalism to higher dimensions Class. Quantum Grav. 27215010

[72] Carot J and da Costa J 1993 On the geometry of warped spacetimes Class. Quantum Grav. 10 461-82

[73] Wald R M 1984 General Relativity (Chicago, IL: The University of Chicago Press)

[74] Procesi C 2007 Lie Groups: An Approach through Invariants and Representations (Berlin: Springer)

[75] Eberlein P and Jablonski M 2009 Closed orbits of semisimple group actions and the real Hilbert-Mumford function Contemp. Math. 491 283-321

[76] Coley A, Hervik S and Pelavas N 2009 Spacetimes characterized by their scalar curvature invariants Class. Quantum Grav. 26025013

[77] Hervik S and Coley A 2010 Curvature operators and scalar curvature invariants Class. Quantum Grav. 27095014

[78] Senovilla J M M 2006 The universal 'energy' operator Class. Quantum Grav. 23 7143-7

[79] Robinson I 1997 On the Bel-Robinson tensor Class. Quantum Grav. 14 A331-A333

[80] Ortaggio M, Pravda V and Pravdová A 2013 Algebraic classification of higher-dimensional spacetimes based on null alignment Class. Quantum Grav. 30013001

[81] Petrov A Z 1969 Einstein Spaces (Oxford: Pergamon) (translation of the 1961 Russian edition)

[82] Hall G 2004 Symmetries and Curvature Structure in General Relativity (Singapore: World Scientific)

[83] Reboucas M J, Santos J and Teixeira A F F 2004 Classification of energy momentum tensors in $n>5$ dimensional space-times: a review Braz. J. Phys. 34 535-43

[84] Bergqvist G and Senovilla J M M 2001 Null cone preserving maps, causal tensors and algebraic Rainich theory Class. Quantum Grav. 18 5299-325

[85] Milson R 2004 Alignment and the classification of Lorentz-signature tensors arXiv:gr-qc/0411036

[86] Coley A, Milson R, Pravda V and Pravdová A 2004 Vanishing scalar invariant spacetimes in higher dimensions Class. Quantum Grav. 21 5519-42

[87] Hawking S W and Ellis G F R 1973 The Large Scale Structure of Space-Time (Cambridge: Cambridge University Press)

[88] Kar S and SenGupta S 2007 The Raychaudhuri equations: a brief review Pramana 69 49-76 